\title{
A revised taxonomy for Uvaria (Annonaceae) in continental Asia
}

\author{
Authors: Meade, Conor V., and Parnell, John A. N.
}

Source: Australian Systematic Botany, 31(4) : 311-356

Published By: CSIRO Publishing

URL: https://doi.org/10.1071/SB17051

BioOne Complete (complete.BioOne.org) is a full-text database of 200 subscribed and open-access titles in the biological, ecological, and environmental sciences published by nonprofit societies, associations, museums, institutions, and presses.

Your use of this PDF, the BioOne Complete website, and all posted and associated content indicates your acceptance of BioOne's Terms of Use, available at www.bioone.org/terms-of-use.

Usage of BioOne Complete content is strictly limited to personal, educational, and non - commercial use. Commercial inquiries or rights and permissions requests should be directed to the individual publisher as copyright holder.

BioOne sees sustainable scholarly publishing as an inherently collaborative enterprise connecting authors, nonprofit publishers, academic institutions, research libraries, and research funders in the common goal of maximizing access to critical research. 


\title{
A revised taxonomy for Uvaria (Annonaceae) in continental Asia
}

\author{
Conor V. Meade (D) A,C and John A. N. Parnell ${ }^{\mathrm{B}}$ \\ AMolecular Ecology Laboratory, Maynooth University Biology Department, Callan Building, Maynooth, \\ Co. Kildare, W23 F2H, Ireland. \\ ${ }^{B}$ Department of Botany, School of Natural Sciences, University of Dublin, Trinity College, D02 PN40, Ireland. \\ ${ }^{\mathrm{C}}$ Corresponding author. Email: conor.meade@mu.ie
}

\begin{abstract}
This paper presents a revision of Uvaria L. (Annonaceae) in continental Asia and outlying islands, north of the Thailand-Malaysia border, on the basis of a combination of new morphological analyses and recent phylogenetic data. Two new taxonomic groupings are defined within the genus in Asia on the basis of detailed morphological character analysis in $\sim 1800$ specimens. Stamen and carpel structure are shown to be significantly more informative than calyx and corolla characters for subgeneric differentiation, and reliably discriminate basal and derived radiations when mapped onto recent molecular phylogenies for the genus in Asia. Thirty-three species and species varieties are recognised within Uvaria in continental Asia, incorporating taxa formerly assigned to Anomianthus Zoll., Cyathostemma Griff., Ellipeia Hook.f. \& Thomson, Ellipeiopsis R.E.Fr. and Dasoclema J.Sinclair. Four new combinations are proposed, and outstanding taxonomic, nomenclatural and typification issues are resolved for included taxa. Keys for both flowering and fruiting material are included, and distribution data are provided for all taxa.
\end{abstract}

Received 30 September 2017, accepted 4 October 2018, published online 6 December 2018

\section{Introduction}

Uvaria L. is the second-largest palaeotropical genus in the Annonaceae (custard apple family) and includes $\sim 150$ species sensu stricto (Kessler 1993) and 210 species sensu lato (Zhou et al. 2010). Uvaria and associated genera have historically formed a well defined stellate-haired taxonomic group, the 'Uvaria-group' (Van Heusden 1992), comprising Uvaria, Balonga Le Thomas (Africa), Anomianthus Zoll. (Asia), Cyathostemma Griff. (Asia), Ellipeia Hook.f. \& Thomson (Asia), Ellipeiopsis R.E.Fr. (Asia) and Rauwenhoffia Scheff. (Asia). However, many inconsistencies exist in nomenclature, taxonomy and typification (Meade 2001; Meade and Parnell 2002). Recent phylogenetic analysis of the Uvaria lineage within the wider Annonaceae has also raised questions regarding differentiation of genera within the Uvaria group, with the implication that a monophyletic Uvaria genus, encompassing all the above genera, is the most appropriate classification (Zhou et al. 2010, 2012; Chatrou et al. 2012).

To redress these issues, the current paper presents a revision of the Uvaria group in continental Asia, covering all taxa that occur from the western Ghats to the Thai-Malay border, just south of the Isthmus of Kra, and including Sri Lanka, the Andaman Islands and Hainan Island in the South China Sea. In support of this revision, key morphological traits are re-evaluated in terms of their taxonomic and phylogenetic utility across the lineage as a whole, with the objective of establishing a revised and more robust taxonomy.

\section{Morphology of the Uvaria group}

Uvaria species occur in the moist tropical regions of Africa, Madagascar, continental Asia, Malesia, northern Australia and the Bouganville-Solomon Island chain. Most species are climbers or scrambling shrubs, and grow by means of twining branchlets, although a small number are self-supporting shrubs. Lowland rainforest species can grow to heights of $30 \mathrm{~m}$ or more, often with a single $8-10-\mathrm{cm}$ diameter main stem; however, the more usual habit is a many-branched climber in disturbed or marginal riverine vegetation, or less often in seasonally dry scrub and deciduous forest.

The general morphology of Uvaria, although consistent across key taxonomic traits such as the possession of stellate hairs, is, nonetheless, quite varied, attendant to the size of the genus and consistent within the broad parameters of the Annonaceae (Fig. 1, 2); the definitions presented in Van Heusden (1992), after Radford et al. (1974), are used here for morphological descriptions and terminology without significant alteration. Although Uvaria taxa in Africa and Asia share core homology across all diagnostic taxonomic traits for the genus (as per the generic description that follows), the analysis and discussion presented here focus on variation in Asian Uvaria taxa, with some additional reference to equivalent observed variation in African taxa, especially regarding inferred patterns of biogeographic radiation and floral character evolution across the genus. 
A

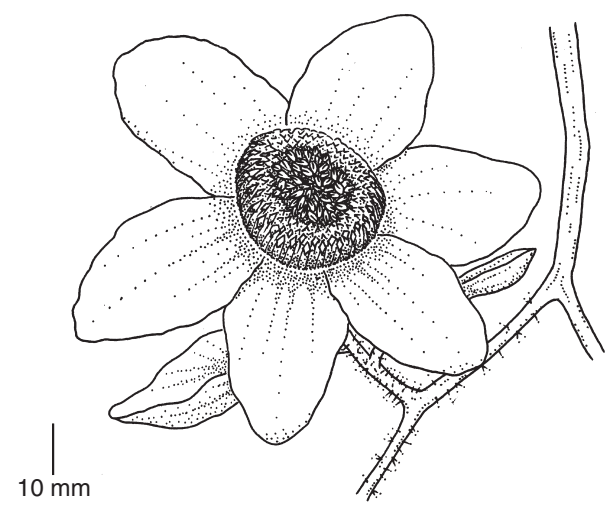

D

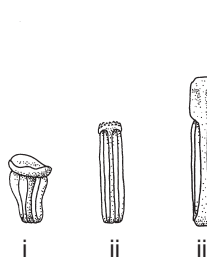

B

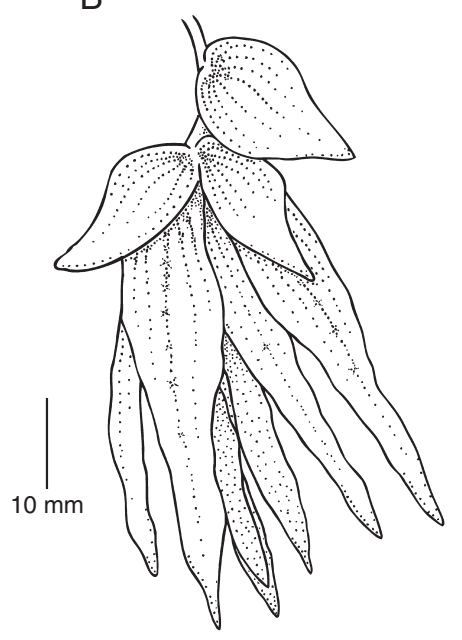

C

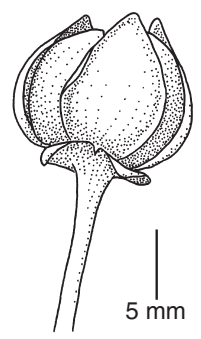

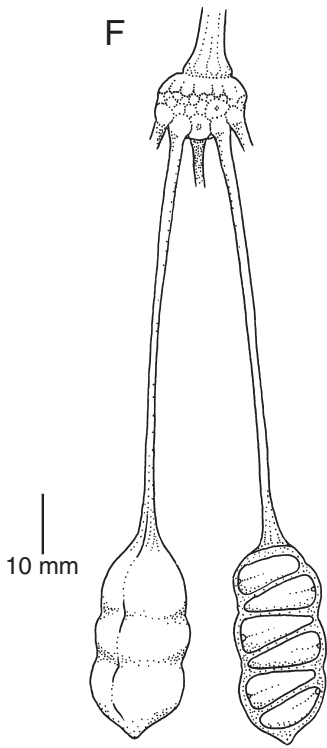

G

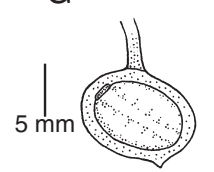

Fig. 1. Morphology of Uvaria group taxa. A. U. grandiflora flower, with spreading petals in two whorls of three. B. U. hahnii flower, with erect petals. C. U. boniana flower, with erect petals. D. Stamen structure: i. U. zeylanica, stamens cuneate in shape, locules subequal, extending full length of connective; ii. U. semecarpifolia, laminar in shape, locules subequal, extending full length of connective; iii. U. rufa, laminar in shape, locules subequal, extending full length of connective, connective apex elongate; iv. U. microcarpa staminode; v. U. hahnii, cuneate in shape, connective apex umbonate, locules unequal in length, raised on a short filament; vi., U. pauciovulata, cuneate in shape, connective apex obtuse, locules unequal in length, raised on a short filament. E. Carpel structure: i. U. dac, ii. U. rufa, iii. U. utteridgei, iv. U. pierrei, v. U. siamensis, vi. U. pauciovulata. F. U. concava mature monocarps, placentation lateral. G. U. pauciovulata mature monocarp, placentation subapical. F. Illustration C. V. Meade.

\section{Inflorescence}

The inflorescence can be leaf-opposed or axillary, and, in rare cases, is cauliflorous. Flowers range in number from one to five or six per inflorescence, with usually a maximum of two or three open at any one time. Among Uvaria taxa, calyx structure presents in three different states, namely, valvate, connate and calyptrate (Fig. 2A), the latter sometimes splitting unevenly into $3+$ sepals. The corolla comprises two whorls of three imbricate petals (Fig. 1A-C), except in the case of four taxa where the petals are basally connate in a single whorl (Fig. 3B). Inner and outer whorls are most often subequal, with the inner petals typically only marginally smaller than the outer ones; however, in a minority of taxa, the inner petals are distinctly smaller. Prior to opening of the floral bud, adjacent petals in each whorl are imbricate along their upper margins; at maturity, the petals form alternate whorls overlap, usually only at the base (Fig. 1A, 4D). In the majority of species, inner- and outerwhorl petals have the same shape and structure. Less often, inner-whorl petals differ from the outer-whorl ones, by carrying basal swellings on the margin, by having a different indument (for example, being basally glabrous on the inner surface) or by having a different shape (for example, by being conspicuously narrower, or where the inner petal margins remain connivent). Petals are typically membranous (Fig. 1A, 5D) or coriaceous (Fig. 1B); however, some Asian and many African species have fleshy petals (Fig. 1C). In some species, the petals remain erect (Fig. 1B), but mostly they are spreading or reflexed at maturity (Fig. 5D). Petal colours range from white to yellow to purple to brown. Torus shape ranges from flat to globose to depressed ovoid.

\section{Stamens}

Stamen structure is highly variable. Stamens are typically laminar (Fig. 1Dii, iii) or cuneate (Fig. 1Dv, vi) and the apex of the stamen connective may be dorsally flattened (Fig. 1Di), blunt (Fig. 1Dii), elongate (Fig. 1Diii) or umbonate (convex) (Fig. 1Dv, vi). Many species have papillae on the stamen apex (Fig. 1Dii, v) and, although most species have glabrous stamens, a few have hairs on the locules (thecae) or on the connective. In the majority of taxa, the filament is rudimentary or absent and all locules extend the full length of the connective from below the apex to the base (Fig. 1Di-iii), less often the 


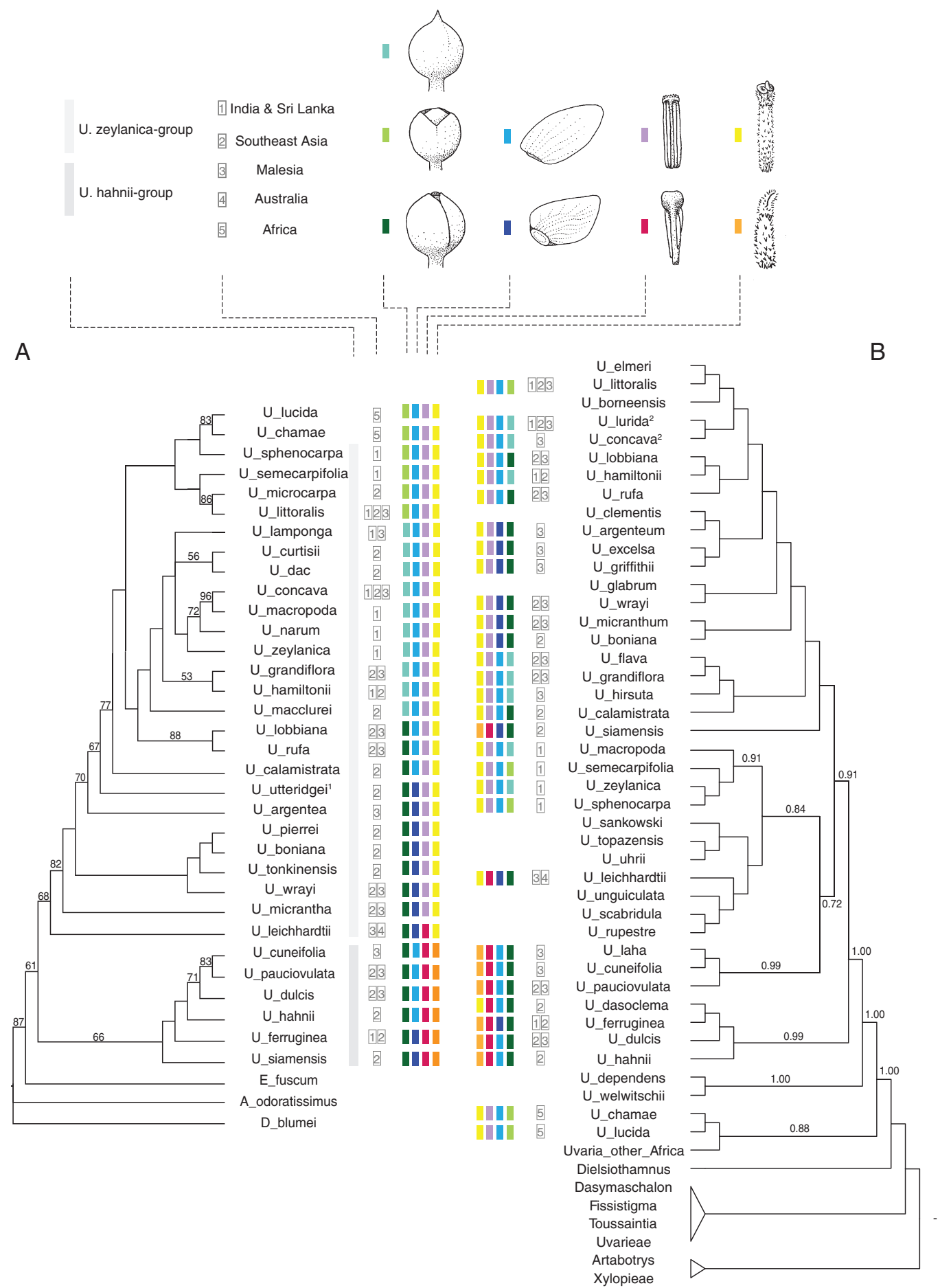

Fig. 2. A. Cladogram of morphometric association among sampled Uvaria group taxa inferred from maximum-parsimony analysis of 55 taxonomic traits $(50 \%$ majority rule consensus tree of 3 most parsimonious trees). Tree length (with weights reset to 1$)=166$, consistency index $(\mathrm{CI})=0.404$, retention index $(\mathrm{RI})$ $=0.708$, and rescaled consistency index $(\mathrm{RC})=0.286$. Bootstrap values above branches were calculated over 1000 repetitions using tree bisection reconnection (TBR). All dichotomous branches have 100\% majority-rule consensus support. B. Maximum clade-credibility tree inferred in BEAST analysis of four plastid DNA regions in the sampled taxa. Branch support values indicate Bayesian posterior probability for each node (values less than 0.95 omitted, except where indicated; after Zhou et al. 2012). The four most informative character states used for the present taxonomic revision are indicated and colour-coded for the surveyed taxa in both trees (calyx in bud calyptrate, connate or valvate; petals membraneous or thickened; stamen filament absent or present; stigma glabrous or hairy); taxa in B without coding were not sampled for the present analysis. Taxon names are listed according to the revised taxonomy proposed in the current paper, with all Uvaria group taxa subsumed into Uvaria. Cyathostemma siamense = Uvaria utteridgei. Uvaria concava and U. lurida are treated as the same taxon in the present revision. 


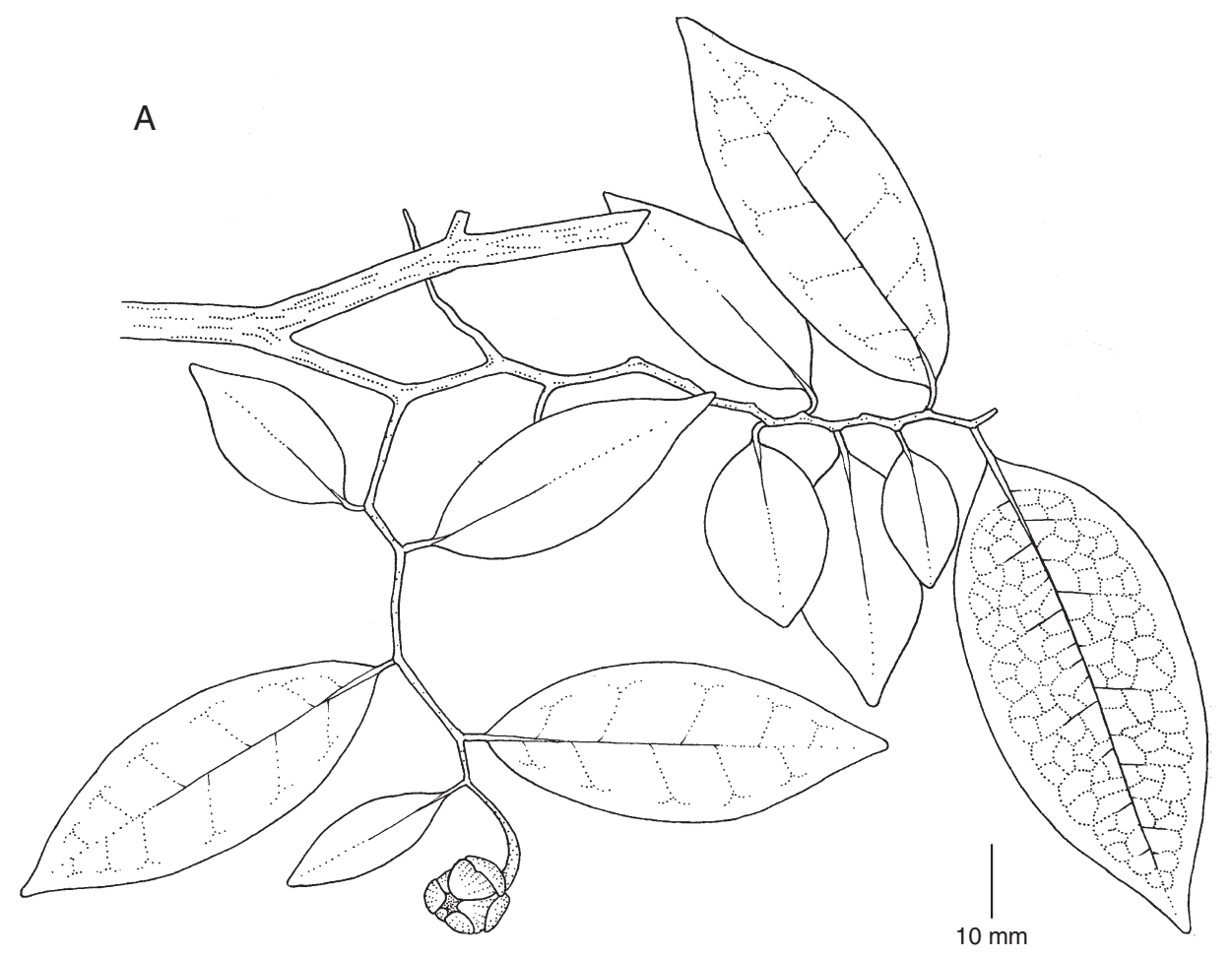

B

C
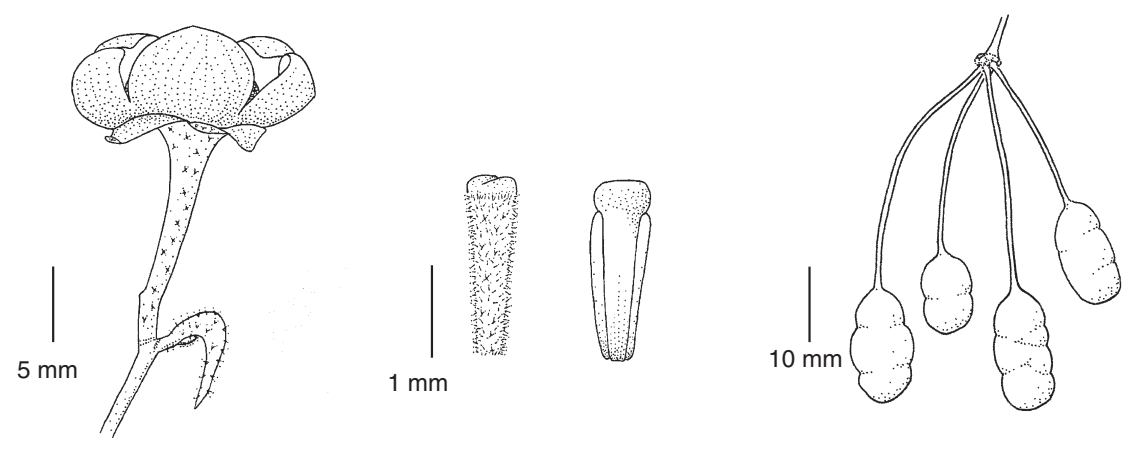

Fig. 3. Uvaria narum. A. Habit. B. Mature flower. C. Carpel and stamen. D. Mature monocarps. Illustration C. V. Meade.

locules are unequal in length and raised from the stamen base on a short filament (Fig. 1Dv, vi). In several species, an outer whorl of staminodes or substaminodes is present (Fig. 1Div, 4B, F).

\section{Carpels and fruit}

Ovaries are always hairy and the stigmas are U-shaped in cross-section and either glabrous (Fig. 1Ei-iv) or more rarely hairy (Fig. 1Ev, vi). In most taxa, there is a slight narrowing of the stigma base where it adjoins the carpel (Fig. 1Ei); however, in a minority of cases, this constriction is pronounced (Fig. 1Ev, vi). Ovule number ranges from 1 to
30, with most species having between 4 and 10 ovules, with lateral placentation (Fig. 1G); however, apical or subapical placentation is also observed. Mature seeds vary in shape according to number of fertilised ovules in the ovary, with lateral compression common in monocarps with multiple ovules (Fig. 1F, G, 4C, 6B); aril shape varies from triangular to bilaterally lobed. Monocarps are normally stipitate (Fig. 1F, 4C); however, in a few species, they are sessile or nearly so. The pericarp can be thin or thick and fleshy, although thinning via desiccation is common at maturity. The flesh is usually white in colour and bittersweet to taste. Monocarp surfaces are variable; the indument most often follows the mature vegetative parts, and can be hairy or 
A

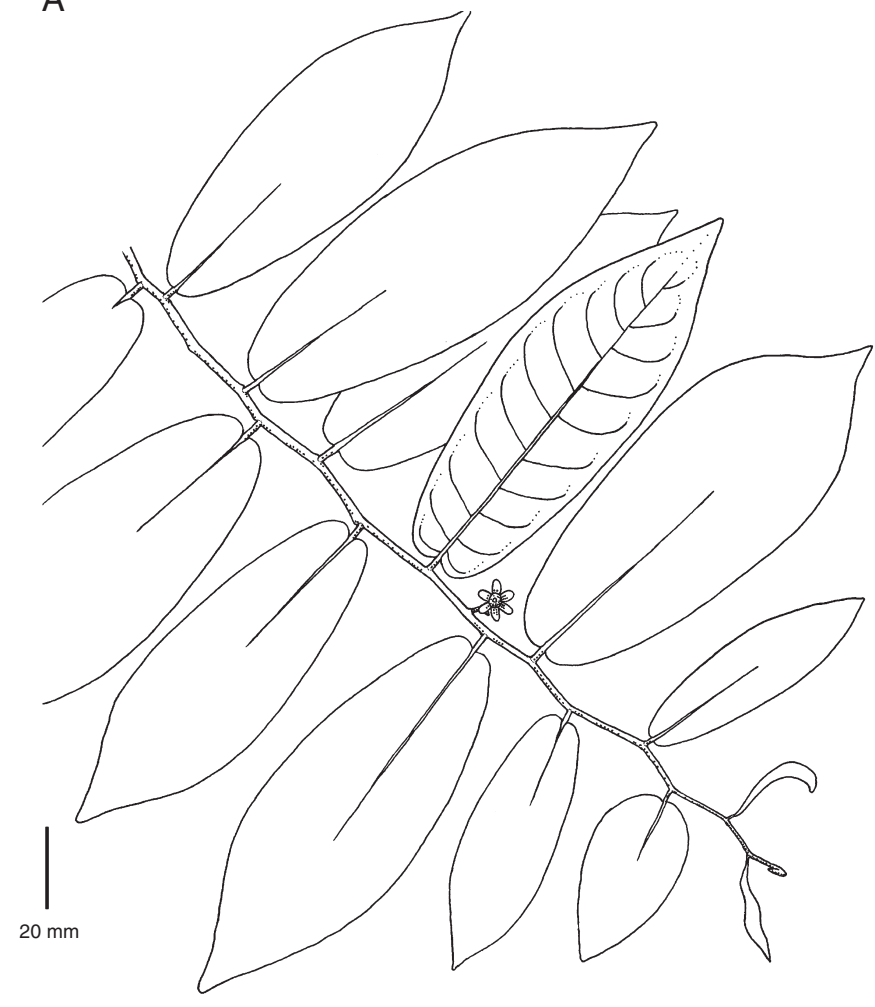

C
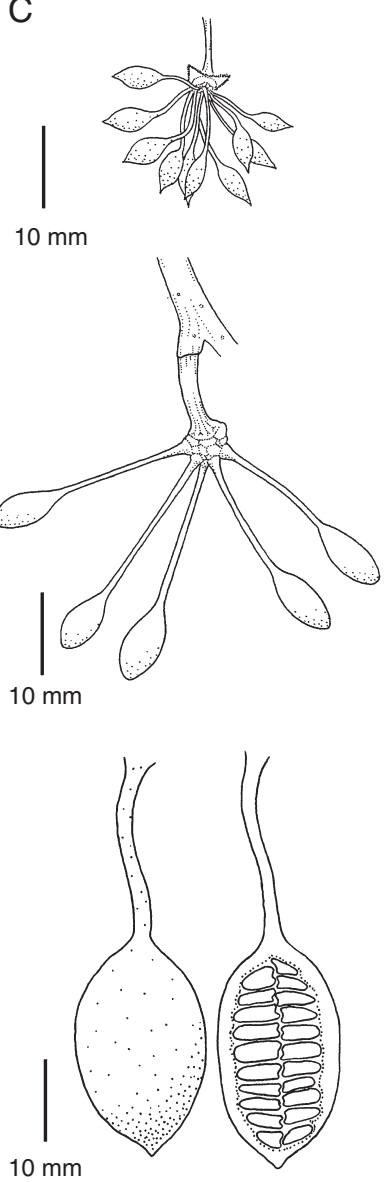

B

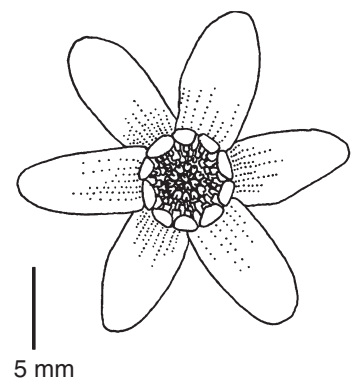

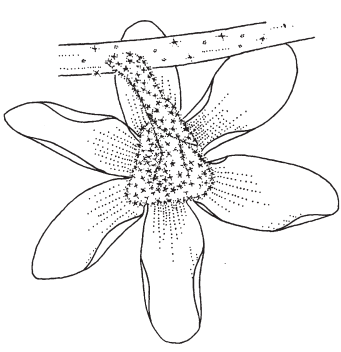

D

E

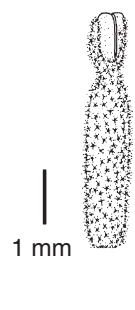

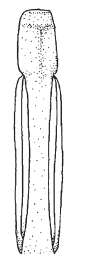

$\mathrm{F}$

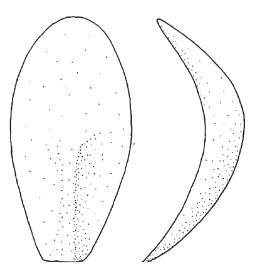

Fig. 4. Uvaria rufa. A. Habit. B. Mature flower. C. Ripening monocarps. D. Carpel. E. Stamen. F. staminode. Illustration C. V. Meade.

glabrous; rarely the fruit indument is long and branched (Meade 2005). Surface features are more independent, and vary from entirely smooth to being prominently tubercled, to having clear longitudinal striation which may be elongate, fine and shallow, or undulating, conspicuous and deep. In many species, a conspicuous dorsal keel and an apicule can persist to maturity (Fig. 1F).

\section{Leaves and indument}

The predominant leaf shape in Uvaria is obovate; however, elliptical and narrowly ovate leaves are also common (Meade and Parnell 2003). The shape of the leaf base varies from cordate (most commonly, for example Fig. 6) to cuneate (Fig. 5, whereas the leaf apex varies from acute (most commonly, for example Fig. 6) to acuminate. Leaves show 6-25+ subparallel veins, depending on the taxon, and although this number can vary with leaf age, it is generally consistent within a narrow range for each taxon (Meade 2001). Most species have a tomentose indument with a characteristic ferruginous colour, especially in the juvenile stages. Many species lose this indument, sometimes very early in development (Fig. 7A), whereas others retain it into 


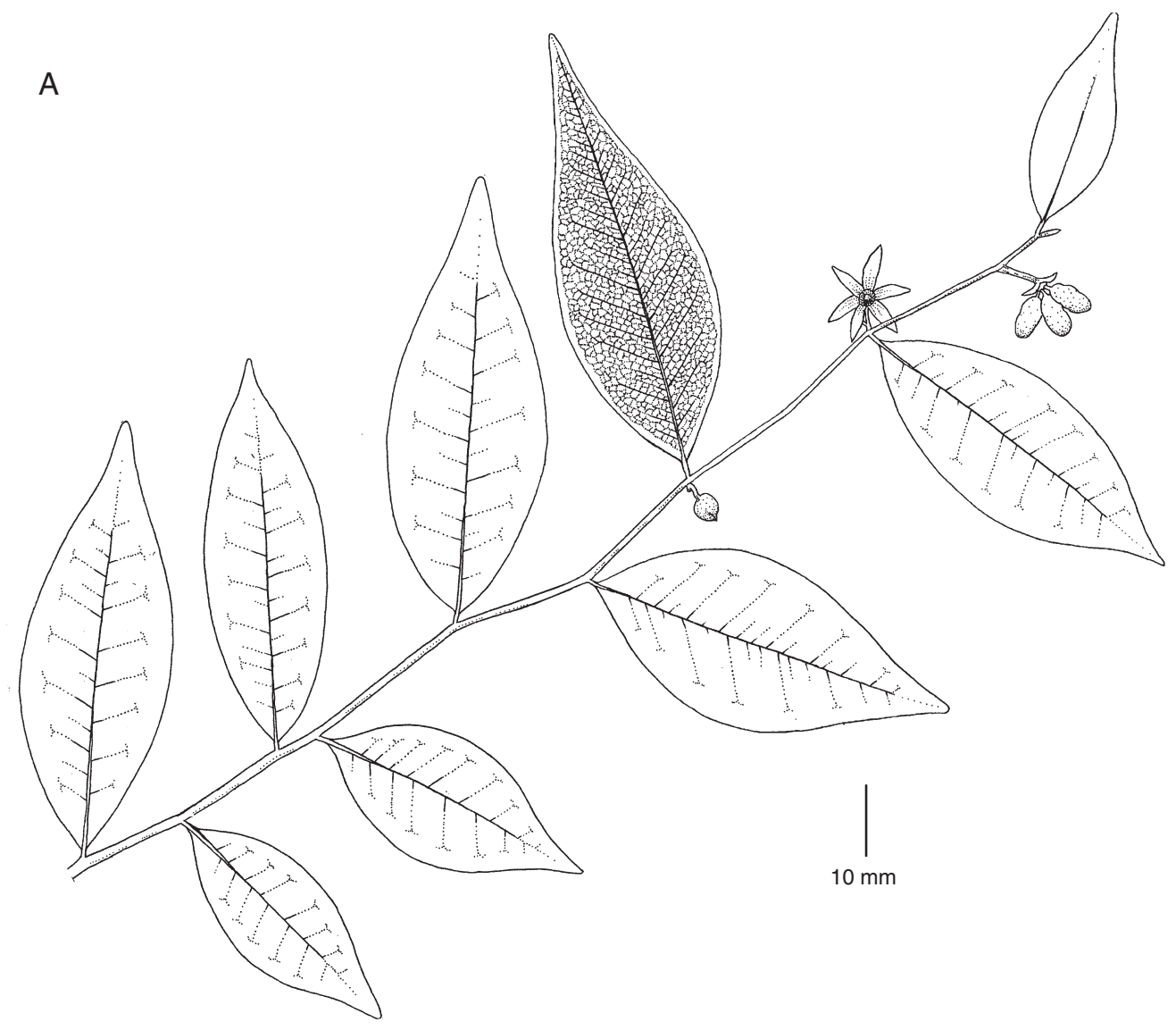

B

C

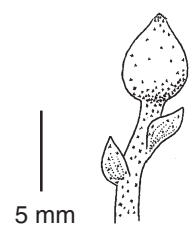

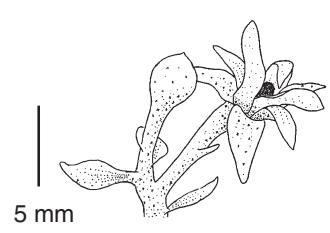

$5 \mathrm{~mm}$

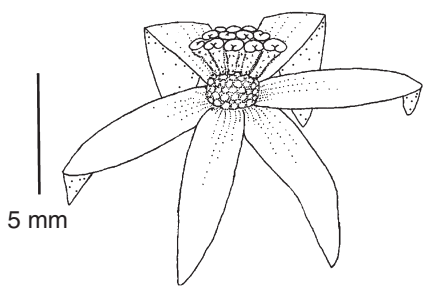

E

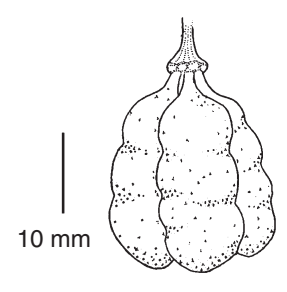

Fig. 5. Uvaria zeylanica. A. Habit. B. Fower bud. C. Flower early anthesis. D. Mature flower. E. Mature monocarps. Illustration C. V. Meade.

maturity, sometimes as a prominent hirsute tomentum (Fig. 6). In all species, the hairs are stellate, with $2-25+$ branches.

\section{Historical treatment of Uvaria}

Delimitation of the Uvaria group within the Annonaceae has focused on the following four main characters: a scrambling or climbing growth habit, stellate hairs over all organs, valvate sepals and imbricate petals. There has been broad taxonomic agreement about the core composition of this grouping within the family (Hooker and Thomson 1855; Finet and Gagnepain 1906; Sinclair 1955; Van Heusden 1992; Meade 2001); however, for example Fries (1959) included many additional genera of less obvious affinity. Disagreement has been greater regarding taxonomic differentiation of core genera within the group, primarily owing to varying emphasis on a variety of petal characters (Fries 1959; Nguyễn 1974, 2000 (often cited by his first name(s) as Bân or Tiễn Bân); Van Heusden 1992; Utteridge 2000). Meade and Parnell (1998), Meade (2001) and Meade et al. (2002) identified strong homoplasy in the 


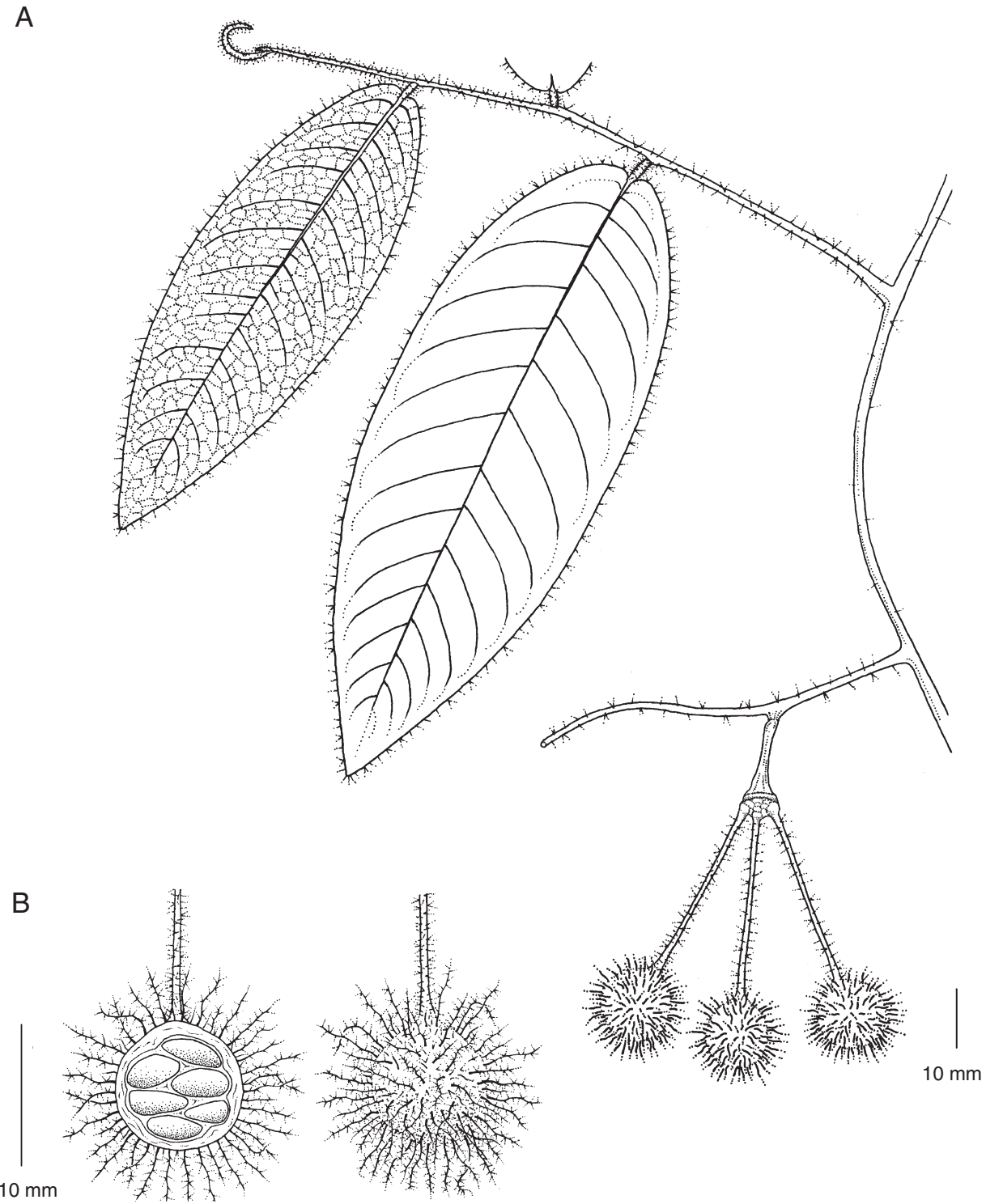

Fig. 6. Uvaria vietnamensis. A. Habit. B. Mature monocarps. Illustration C. V. Meade.

conspicuous floral traits most often used to discriminate among Uvaria group genera, whereas leaf shape and fruit structure appear to be reliable indicators only at species level (Meade 2001; Meade and Parnell 2003).

Early classifications of the Annonaceae, such as those of de Candolle (1824) and Hooker and Thomson (1855), described Uvaria with its multi-ovuled numerous free carpels and spreading petals as the most 'simple' floral arrangement in the family, akin to the Ranunculaceae and Magnoliaceae (Fig. 1A). The later phylogenetic interpretations of Sinclair (1955), on the Malaysian Annonaceae, and Fries (1959), on the entire family, also placed Uvaria in a primitive complex, with Sinclair (1955, p. 169) commenting as follows: 'There is a tendency to proceed from simplicity to complexity, e.g. from simple petals with no distinction between blade and claw, to the complex dome-shaped petals of the Mitrephoreae...'.

\section{Phylogenetic analysis}

Molecular phylogenetic analysis of the Annonaceae has shown more complex floral evolution patterns than envisaged by these earlier workers, with both convergence and reversion of floral traits being displayed across the family, including the traits that delimit the Uvaria group (Saunders 2010, 2012; Couvreur et al. 2011; Chatrou et al. 2012; Thomas et al. 2015). In addition, Uvaria is not placed among the basal Annonaceae genera of origin $c$. 90 million years ago, but in the more derived lineage of the Annonoideae, or Long Branch Clade, which first arose in the late Cretaceous c. 65 million years ago (Couvreur et al. 2011; Chatrou et al. 2012). Plastid DNA evidence also suggests the Uvaria lineage first emerged in Africa during the mid-to-late Oligocene (c. 25-30 million years ago) and later dispersed to Asia from an East African Uvaria clade during the early Miocene (c. 20 million years ago; Couvreur et al. 2011; Zhou et al. 2012). Despite these recent origins within the Long 


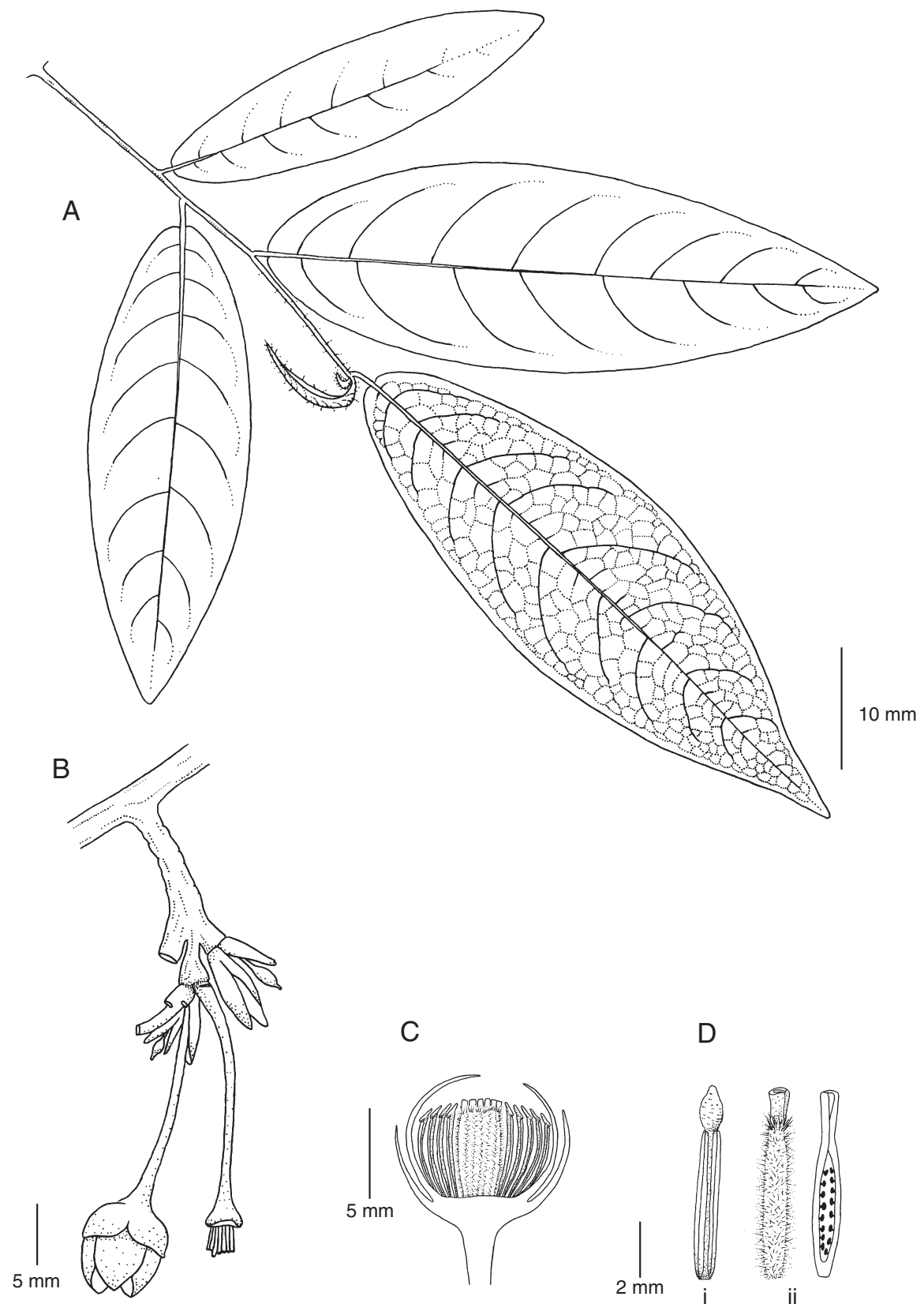

Fig. 7. Uvaria utteridgei. A. Habit. B. Inflorescence. C. Flower, section. D.i. Stamen. ii. Carpel. Illustration C. V. Meade.

Branch Clade (LBC), Uvaria is the third most species-rich genus in the Annoaceae, and on the basis of a sensu lato interpretation of Zhou et al. (2009), displays an elevated speciation rate compared with genera in the family as a whole (Couvreur et al. 2011). While this elevated speciation rate is partly attributable to the biogeographic radiation of Uvaria from Africa into Asia, it also reflects the sensu lato interpretation of Zhou et al. (2009), wherein all the Uvaria group genera of Van Heusden (1992) and Meade (2001) are subsumed into Uvaria on the principle of generic monophyly.

In many branches of the Annonaceae, discrete differences in taxonomy and morphology readily distinguish adjoining monophyletic clades, and allow the maintenance of multiple smaller genera with much more recent origins than Uvaria (Couvreur et al. 2011; Chatrou et al. 2012). In the Uvaria lineage, by comparison, the conspicuous floral traits that have been used in taxonomic discrimination appear to map very poorly to the molecular phylogeny, especially among Asian taxa, and have encouraged strongly paraphyletic classifications for Uvaria group genera (Zhou et al. 2009). However, the resulting proposal that Uvaria group genera be incorporated into Uvaria s.l. has the dual effect not only of eliminating this paraphyly, but also greatly increasing the range of floral and fruit character diversity within the genus 
(Fig. 1A-C). Monophyletic classifications based solely on molecular evidence have the potential to create taxonomic groupings that relate poorly to the (dis)continuity of morphology states within lineages (Kluge 1988), and the inclusion of associated genera into Uvaria, as above, raises this problem.

\section{A revised taxonomy}

Our objective here has been to prepare a taxonomic account of Uvaria in Asia that is congruent with inferred molecular phylogeny and of practical use to botanists in the field. First, to resolve existing confusion, we have re-evaluated the morphological utility of floral, fruit and vegetative traits that have traditionally been used for taxonomic discrimination across the Uvaria group, using a cladistic approach. Key character states that show robust informativeness are then mapped to the chloroplast phylogeny to determine which best mark out the inferred history of the Uvaria lineage. We then propose a revised taxonomy and key for Uvaria, incorporating new subdivisions demarcated by discriminating morphological traits that map to defined nodes on the chloroplast phylogeny, and, on this basis, we present a taxonomic revision for the genus in continental Asia.

\section{Analysis of floral character evolution in Uvaria}

\section{Materials and methods}

\section{Data generation}

A morphological-character binary-data matrix was generated from 55 characters scored from 37 taxa in the Uvaria group (Table 1, Table S1, available as Supplementary material to this paper), encompassing the measurable variation in morphology observed for the genus, as introduced above. Identification of variable traits was completed first in consulation with type material, and later validated across the observed variation in non-type collections. In the completed analysis, six characters were based on continuous count or size measurements, for which character states were determined using ratio comparisons (e.g. leaf length to width ratio), relational comparisons or statistical variance (e.g. in which a sample score is inside (0) or outside (1) one standard deviation of the sample mean). The remaining 49 characters were scored on the basis of discrete presence or absence traits. Traits $1-6$ addressed variation in vegetative morphology (Table 1); Traits 7-10, inflorescence structure; Traits 11-27 and Traits 53-55, calyx and corolla structures; Traits 28-33, 46 and 51, carpel structure; Traits 34-39, stamen structure; and Traits 41-49 and 52, fruit and seed structure.

Thirty taxa from Uvaria and Cyathostemma were sampled, along with representatives from Anomianthus, Ellipeia, Ellipeiopsis and Rauwenhoffia (all putative members of the Uvaria group on the basis of morphology), plus the stellatehaired Melodorum Hook.f. \& Thomson of Malesia and northern Australia. Dasymaschalon Dalla Torre \& Harms (Annonoideae: Uvarieae), Artabotrys R.Br. (Annonoideae: Xylopieae) and Enicosanthum Becc. (Malmeoideae: Miliusiae) were sampled as outgroups, on the basis of their inferred floral dissimilarity or similarity with Uvaria, after Sinclair (1955) and Van
Heusden (1992). Approximately 1800 specimens (including most of the relevant types) were examined from the following herbaria: A, AAU, ABD, BK, BKF, BM, BR, C, CMU, E, G, HAM, PHH, HN, HNU, K, L, LIV, LISU, NY, P, PE, SING, TCD, U, US. In the main text accounts, barcodes are quoted for specimens studied, where available. The full list of examined material is included in the 'Full list of material examined' section in the Supplementary material.

\section{Data analysis}

Cladistic analysis of the morphological data matrix were performed using maximum-parsimony (MP) criteria (Edwards and Cavalli-Sforza, 1964; Camin and Sokal 1965) as implemented in PAUP (ver. 4.0b10. D. W. Swofford, Sinaur Associates, Sunderland, MA, USA; Swofford 2002). Maximumparsimony analysis comprised an initial heuristic search using random stepwise addition and the tree bisection and reconnection (TBR) branch-swapping algorithm, followed by the same search with characters reweighted to their mean rescaled-consistency $(\mathrm{RC})$ index score from the first analysis. Bootstrap values were calculated over 1000 iterations on these reweighted data by using TBR.

\section{Results}

Maximum-parsimony analysis of the entire sample dataset yielded three most parsimonious topologies, with a consistency index (CI) score of 0.404 (with character weights reset to 1 for the purpose of tree interpretation), a retention index (RI) score of 0.708 and an RC score of 0.286 (50\% majority-rule consensus topology shown in Fig. 2A). A total of 11 characters had mean RC index scores of 1.0 and changed state just once on the topology (Table S2, available as Supplementary material to this paper). Five of these characters relate to petal structure $(18,22,27,53,54)$, three to stamen structure $(34,35$, $38)$, two to carpel structure $(31,33)$ and one to indument structure (50). One petal character (24) had an RC score of $>0.5$, whereas all the remaining characters (including all fruit, leaf and inflorescence characters) had scores of $<0.5$.

The inferred tree topologies show a basal morphometric division between a group containing Anomianthus, Ellipeia, Ellipeiopsis, Rauwenhoffia, Uvaria hahnii (Finet \& Gagnep.) J.Sinclair and U. pauciovulata Hook.f. \& Thomson (defined by a convex or umbonate connective apex in the stamen and a hairy stigma, termed here the ' $U$. hahnii-group', 66\% bootstrap support, Fig. 2A) and a larger group containing all the remaining Uvaria and Cyathostemma taxa and Melodorum leichhardtii (F.Muell.) Benth. (connective apex not convex or umbonate, stigma glabrous, the ' $U$. zeylanica-group', $68 \%$ support). Bootstrap support for the ' $U$. zeylanica-group' above M. leichhardtii is $82 \%$.

The following three major subgroupings within the ' $U$. zeylanica-group' can be delimited using calyx structure: the terminal group above and including Uvaria macclurei Diels (calyx calyptrate, Character 13), the terminal group including $U$. littoralis Blume and U. chamae P.Beauv. (calyx connate, Character 11) and the remainder of the taxa on the tree (calyx valvate, Character 12). The only characters that robustly discriminate at the base of the topology are an umbonate 
Table 1. Data scoring criteria for 55 morphological character states among Uvaria taxa, as applied in the presented cladistic analysis

Trait states have been demarcated on the basis of observed variation in type-specimen morphology and associated non-type collections. See text for discussion

\begin{tabular}{|c|c|c|c|c|c|}
\hline \multicolumn{2}{|c|}{ Character } & \multirow{2}{*}{$\begin{array}{l}0 \\
\leq 1 \mathrm{~mm}\end{array}$} & \multirow{2}{*}{$\frac{1}{\geq 1 \mathrm{~mm}}$} & \multirow[t]{2}{*}{2} & \multirow[t]{2}{*}{3} \\
\hline 1 & Shoot indument & & & & \\
\hline 2 & Leaf $\mathrm{L}: \mathrm{W}$ ratio & $\leq 2.07$ & $2.08-2.44$ & $2.45-3.07$ & $\geq 3.07$ \\
\hline 3 & Upper blade glabrous & No & Yes & & \\
\hline 4 & Upper blade scabrous & No & Yes & & \\
\hline 5 & Secondary veins & Complete & Incomplete & & \\
\hline 6 & Leaves & Coriaceous & Membranous & & \\
\hline 7 & Inflorescence & Subterminal & Terminal & & \\
\hline 8 & Pedicel transverse section & Circular & Flattened & & \\
\hline 9 & Torus diameter & $\leq 5 \mathrm{~mm}$ & $>5 \mathrm{~mm}$ & & \\
\hline 10 & Torus & Raised & Flat & & \\
\hline 11 & Calyx connate & No & Yes & & \\
\hline 12 & Calyx valvate & No & Yes & & \\
\hline 13 & Calyx calyptrate & No & Yes & & \\
\hline 14 & Sepals & Flat & Concave & & \\
\hline 15 & Calyx & Smooth & Tuberculate & & \\
\hline 16 & Calyx apicule & No & Yes & & \\
\hline 17 & Calyx splitting & Evenly & Unevenly & & \\
\hline 18 & Petals variable in number & No & Yes & & \\
\hline 19 & Petals membranous & No & Yes & & \\
\hline 20 & Petals thickened & No & Yes & & \\
\hline 21 & Whorls & Equal & Outer $>$ inner & & \\
\hline 22 & Inner whorl base & Hairy & Glabrous & & \\
\hline 23 & Inner whorl base & Obtuse & Narrowed & & \\
\hline 24 & Inner petals & Reflexed & Erect & Closed & Conniven \\
\hline 25 & Marginal glands & Absent & Present & & \\
\hline 26 & Petal colour & Red or purple & Yellow or white & & \\
\hline 27 & Petal base at maturity & Free & Connate & & \\
\hline 28 & Stigma & Short & Elongate & & \\
\hline 29 & Stigma & Conical & Not so & & \\
\hline 30 & Stigma lobes & Narrow & Broad & & \\
\hline 31 & Stigma & Glabrous & Hairy & & \\
\hline 32 & Neck at apex of ovary & No & Yes & & \\
\hline 33 & Neck at stigma base & No & Yes & & \\
\hline 34 & Stamens all fertile & No & Yes & & \\
\hline 35 & Substaminodes & Absent & Present & & \\
\hline 36 & Locules & Glabrous & Hairy & & \\
\hline 37 & Connective apex & Papillate & Smooth & & \\
\hline 38 & Connective apex & Not convex & Convex & & \\
\hline 39 & Locules raised & No & Yes & & \\
\hline 40 & Carpel length & $<$ Stamen & $=$ Stamen & >Stamen & \\
\hline 41 & Fruit stipe length & Sessile & $1 \leq x \leq 30 \mathrm{~mm}$ & $30<x \leq 55 \mathrm{~mm}$ & $>55 \mathrm{~mm}$ \\
\hline 42 & Stipe thickened & No & Yes & & \\
\hline 43 & Fruit $\mathrm{L}: \mathrm{W}$ ratio & $x<1$ & $1 \leq x<2$ & $2 \leq x<3$ & $x=3+$ \\
\hline 44 & Monocarp keel or ridge & Absent & Present & & \\
\hline 45 & Monocarp apicule & Absent & Present & & \\
\hline 46 & Seed number & 1 & $2-16$ & $16+$ & \\
\hline 47 & Pericarp (dried) & Tight & Loose & Thick & \\
\hline 48 & Aril & Triangular & Lobed & & \\
\hline 49 & Seed orientation & Perpendicular & Angled & & \\
\hline 50 & Hairs & Stellate & Simple & & \\
\hline 51 & Ovaries & Basal & Lateral & Apical & \\
\hline 52 & Monocarps & Whole & Moniliform & & \\
\hline 53 & Petals & Imbricate & Valvate & & \\
\hline 54 & Petals & Not clawed & Clawed & & \\
\hline 55 & Sepals & Valvate & Imbricate & & \\
\hline
\end{tabular}


connective apex and a hairy stigma (Characters 31 and 38; Fig. 1Dv, vi, Ev, vi, 2A).

\section{Discussion}

\section{Maximum-parsimony analysis of morphometric trait} variation in the Uvaria group

The goal of this analysis was to identify, from among the range of traits used traditionally by botanists, the characters with the most reliable discriminatory power for the purposes of taxonomic classification. These analyses returned a consensus topology (Fig. 2A) that shares close similarity with topologies inferred in molecular phylogenetic analyses of the Uvaria group (Fig. 2B, after Zhou et al. 2012; Zhou et al. 2009, 2010; Chatrou et al. 2012), a basal cluster of species associated with $U$. hahnii and $U$. pauciovulata, and the remainder of taxa placed above this group. As previously identified by Van Heusden (1992, for stamens and carpels) and Tsou and Johnson (2003, for stamens), androecium and gynoecium traits here emerge as characters of key discriminating importance.

While molecular data carries the determining phylogenetic signal for systematic analysis, and direct trait-mapping yields taxonomies that are entirely in keeping with the inferred DNA topology, the analyses presented here have independently identified morphological patterns that align well with the DNA topology, suggesting that robust morphometric evaluation can identify reliable discriminating apomorphies within this Annonaceae lineage. The CI score of 0.404 indicate that there is a large amount of character homoplasy in the data matrix, which is not uncommon for morphological data. However, a RI score of 0.708 indicates that although there is a large number of character changes in the topology, the number of changes observed is considerably smaller than the total number that are possible. Leaf characters emerge as having poor information value in the dichotomous topology, although, as already establised by Meade and Parnell (2003), multivariate methods can more reliably exploit continuous leafshape characters for taxonomic discrimination.

In terms of using this morphological trait analysis to inform a new taxonomy and key, the following three principal morphological groupings are evident (Fig. 2A): (1) a large terminal group including Asian and African Uvaria species with coriaceous petals, (2) an intermediate group with mostly thickened petals, including $M$. leichhardtii and $U$. boniana Finet \& Gagnep., and (3) a basal group that includes Ellipeia, Rauwenhoffia, Ellipeiopsis and Anomianthus, as well as $U$. pauciovulata and $U$. hahnii. Enforcing a stricter interpretation based only on the most reliable characters, namely stamen and carpel structure, shows only one major division in the cluster, namely, between the basal U. hahnii group and the remaining $U$. zeylanica group, including all taxa above $M$. leichhardtii, and the type species for the genus, $U$. zeylanica L. Notably, solely on the basis of the surveyed characters, the African U. lucida Bojer ex Sweet and U. chamae are clearly nested within the $U$. zeylanica group. The intermediate state of $M$. leichhardtii in terms of stamen structure between the two groups is not wholly reconciled in this (Asian) dataset; however, it seems likely, on the basis of this anomaly, that the wider group of Melodorum taxa from Malesia-Australia also embody a distinct morphological identity within the Uvaria lineage.

\section{Chloroplast phylogeny of the Uvaria group}

The data of Zhou et al. (2009, 2010, 2012) and Chatrou et al. (2012) suggest that the entire 'Uvaria group' in Asia appears to have radiated from a single dispersal event from Africa, implying (1) that Uvaria sensu stricto is paraphyletic, and (2) that the African and Asian lineages of Uvaria are not monophyletic sister groups (Fig. 2B).

The data also suggest that the following three monophyletic clades exist in the Asian Uvaria lineage: (1) a crown group comprising Rauwenhoffia, Cyathostemma and the majority of Uvaria species from South-east Asia and Malesia, (2) a basal group comprising Anomianthus, Ellipeiopsis and U. hahnii, along with the monotypic genus Dasoclema and (3) an intermediate clade comprising U. pauciovulata and all Uvaria species from Peninsular India and Sri Lanka, plus Ellipeia, and Melodorum from Malesia-Australia (Fig. 2B).

Although these inferred phylogenies differ from the established taxonomy for the Uvaria group, the plastid data contain a strong biogeographic signal, implying several region-specific radiation events in the Asian Uvaria lineage, including in India, in South-east Asia, in Malesia and in Australia. These events are consistent with a gradual colonisation of Asia by a Uvaria lineage that first emerged in East Africa, and later developed multiple region-specific morphological novelties as it dispersed across the heterogeneous landscape of southern and south-eastern Asia during the Miocene (24-5.5 million years ago) and Pliocene (5.5-2.5 million years ago). Implicit in this chloroplast data is that the calyx and corolla characters (principally sepal and petal thickness and reflexion, and the presence of marginal glands), which have traditionally been used to delineate genera in the Uvaria group, are homoplasic, and distinguish only apomorphic variants of an ancestral form, a pattern of character-trait distribution that also emerges in the morphometric analysis presented here.

Adhering to the principal of monophyly and assigning all the Uvaria group taxa to a larger Uvaria s.l. presents a basic problem for the taxonomist because this monophyletic group contains several morphological anomalies, notably the divergent floral types seen among the basal Asian clades, compared with the more derived type inherent in the original generic account (Linnaeus 1753).

Some underlying patterns provide a basis for a new taxonomy. Although branch support for terminal clades in the analyses of Zhou et al. $(2009,2010,2012)$ is reduced, compared with more basal vicariance events in these phylogenies, in two cases, the basal member of the terminal clade displays raised stamen locules and a hairy stigma, whereas those in crown branches of the clade do not (Fig. 2B). This pattern implies that regional expansion events have given rise to multiple separate apomorphic changes to an ancestral Asian floral structure. It is likely that this ancestral floral type is the one retained by taxa in the basal Uvaria hahnii clade, because the alternative, namely that the 
U. hahnii-group floral morphology is derived, would imply not two reversals (to the African type, in the clades containing U. leichardtii and U. siamensis (Scheff.) L.L.Zhou, Y.C.F.Su \& R.M.K.Saunders, Fig. 2B), but four independent convergent apomorphies (to the $U$. hahnii-group type, including the $U$. hahnii and $U$ pauciovulata clades). Thus, the Asian ancestral type is likely to differ from its African equivalent, where the inferred ancestral morphology, as evident, for example, in $U$. lucida, matches the more derived Asian type, such as the genus type species, U.zeylanica, and the widespread $U$. littoralis and $U$. grandiflora Roxb. ex Hornem. The positioning of the sampled African U. lucida and U. chamae in the Uvaria group of the morphological analysis captures this similarity, adding weight to the molecular inference that the classic Asian Uvaria morphology is a reversion to an ancestral African form.

Although the various chloroplast lineages of Uvaria in Asia contain species that display both plesiomorphic and apomorphic structural traits (Fig. 2B), the stamen and carpel characters shared by basal members of the principal clades are also the characters that unite the basal clade in the morphometric topology inferred in Fig. 2A. Thus, although several taxa in the chloroplast phylogeny, notably $U$. pauciovulata and $U$. siamensis, emerge as basal to lineages that contain species with very different floral morphologies (Fig. 2B), they appear to retain plesiomorphic character traits associated with the earliest biogeographic expansion of Uvaria into Asia.

\section{A revised taxonomy for Uvaria in Asia}

The above chloroplast phylogeny analyses are unlikely to be final, and this is appropriate. Diagnostic and cladistically robust morphometric traits such as, in this analysis, stamen and carpel structure, may reflect more wide-ranging genomic affinities among Uvaria group taxa that are not captured in the chloroplast haplotype data of Zhou et al. (2009, 2010, 2012). Specifically, analysis of nuclear genomic affinities in Uvaria s.l. will provide a more holistic appraisal of species relations, allowing an inferred phylogeny with clearer links both to adaptive traits and to neutral morphological loci that have been subject to genetic drift (Hartl and Clark 2007; Knowles and Carstens 2007). For the present, these chloroplast data are the best available phylogenetic information for the Uvaria group, and it is reasonable to try and impose a primary monophyletic classification based on it, with additional detail that reflects diagnostic morphological differences among the major groups of taxa.

A monophyletic classification of Uvaria can be implemented by either splitting Uvaria into at least five separate genera (two in Africa, three in Asia), or subsuming all these associated genera into a broadened Uvaria. Although the data presented here suggest that the former would work for Asian taxa, there would be no taxonomic justification for separating Uvaria taxa in continental Africa from the more derived taxa in Asia, such as $U$. zeylanica and $U$. hamiltonii, and this approach would simply create a new paraphyletic classification. Thus, the latter option, creating a larger monophyletic genus, presents the fewest difficulties.
On the basis of this interpretation, and following Zhou et al. (2009), the defining morphological characteristic of the Uvaria lineage within the Annonaceae is the combined presence of stellate (star-shaped) hairs, a valvate calyx and an imbricate corolla. Within this monophyletic genus, and following the analysis presented here, the principal taxonomic division among the Uvaria of Asia rests on the presence or absence of raised stamen locules and an umbonate connective apex, and a hairy stigma. The following two taxonomic groupings are recognised on this basis: the $U$. hahnii group, which display the latter character traits; and the U. zeylanica group, the species in which display full-length locules, a flattened or elongate connective and a glabrous stigma. The dichotomy based on this division presents a conflict in terms of Uvaria siamensis and $U$. pauciovulata (and, although not included in this treatment, $U$. cuneifolia and affiliates of western Malesia) in that these taxa emerge as basal to the Uvaria lineage above the $U$. hahnii group in the chloroplast phylogeny. However, in terms of floral morphology, these species differ markedly from the remaining Uvaria species, although retaining the key traits of $U$. hahnii group that characterise the earliest colonial Uvaria lineages in Asia, as inferred by the molecular data. Considering this clear discontinuity in morphological identity, they are assigned for identification purposes to the $U$. hahnii taxonomic group.

The account presented here includes only a subset of Uvaria taxa that occur in Malesia and Australia (i.e. those species the distribution of which also extends into Peninsular Thailand, as detailed, where relevant, in the maps that follow). Importantly, because of the incomplete sampling of Malesian taxa, the two principal taxonomic groups described here are not proposed formally as sections; however, this may be appropriate with fuller evaluation of morphological diversity across Malesian taxa.

With this revised system, Uvaria is still unlikely to be confused with other Asian Annonaceae, because the only genera that also display stellate hairs are in other ways distinct. In Cyathocalyx Champion ex Hook.f. \& Thomson, the petals are narrowly constricted and elongate above the base, whereas in Anaxagorea Saint Hilaire, Neo-Uvaria AiryShaw and the introduced Annona squamosa L. the ovules have basal placentation. Anaxagorea also displays an inner whorl of staminodes and there are two ovules only. In Africa, Dielsothamnus R.E.Fr. is distinct from Uvaria, in having valvate petals and a single carpel, Annickia van Setten \& Maas has no outer whorl of petals and a single basal ovule, whereas Tetrameranthus R.E.Fr. has eight petals in two whorls and an elongate cylindrical torus. In the Americas, where Uvaria is absent, the stellate-haired Duguetia Saint Hilaire and Pachypodanthium Engler \& Diels are both distinct in having a single basal ovule.

$$
\text { Uvaria L., Sp. pl. 1: } 536 \text { (1753) }
$$

Type: Uvaria zeylanica L.

Marenteria Noronha ex Thouars, Gen. Nova Madagasc. 18 (1806).

Type: not designated.

Cyathostemma Griff., Not. Pl. Asiat. 4: 707, t 650 (1854).

Type: Cyathostemma viridflorum Griff. (as 'viridiflora'). 
Ellipeia Hook.f. \& Thomson, Fl. Ind. 1: 104 (1855).

Type: Ellipeia cuneifolia Hook.f. \& Thomson.

Tetrapetalum Miq., Ann. Mus. Bot. Lugduno-Batavi. 2: 1 (1865).

Type: Tetrapetalum volubile Miq.

Armenteria Baill., Bull. Mens. Soc. Linn. de Paris 1: 338 (1882), nom. inval., pro syn. Rauwenhoffia Scheff., Ann. Jard. Bot. Buitenzorg 2: 21 (1885).

Type: Rauwenhoffia siamensis Scheff.

Uvariella Ridl., Fl. Malay. Penins. 1: 35 (1922).

Type: Uvariella leptopoda (King) Ridl.

Dasoclema J.Sinclair, Gard. Bull. Singapore 14: 273 (1955).

Type: Dasoclema siamensis (Craib) J. Sinclair.

Ellipeiopsis R.E.Fr., Ark. Bot. ser. 2, 3: 41 (1955).

Type: Ellipeiopsis ferruginea (Ham. ex Hook.f. \& Thomson) R.E.Fr.

Habit climbers or sarmentose shrubs, with often prominent ferruginous stellate indument. Leaves membranous or coriaceous, obovate or less often elliptic or ovate, base cordate, subcordate or cuneate, apex acute, acuminate or obtuse, veins 6-25+, subparallel or convergent, secondary veins complete and kinked or incomplete, indument (sub)tomentose and ferruginous on both surfaces of young leaves, in mature leaves abaxial surface sparse or glabrous, hairs erect when persistent, rarely scabrous, adaxial surface tomentose to subglabrous, hairs spreading. Petiole with abaxial groove, adaxial surface often transversely foliated, tomentose to subglabrous. Inflorescence a monochasial or rarely branched rhipidium of 1-5 flowers, typically on younger shoots, rarely cauliflorous, secondary buds arising from prophyll in axil of bract just below articulation of pedicel, bract also present midway along pedicel, bracts ovate or obovate, membranous or coriaceous, clasping or free, sometimes large and leaf-like, often wanting, peduncle, pedicel and calyx with identical indument, hairs tomentose or rarely sparse, fine or warted. Calyx valvate, basally connate or calyptrate, sepals 3 , rarely 2 or 4 , membranous and spreading or fleshy and concave. Corolla petals in 2 imbricate whorls of 3 petals each, petals 6 in number or very rarely rarely 4-8, whorls subequal, less often inner petals conspicuously smaller than and distinct in shape compared with outer, mature petals erect, reflexed or partially reflexed at maturity, fleshy or membranous, ovate, oblong or obovate, red, purple, yellow, white or green, apex obtuse, acute or bluntly acute. Stamens laminar or rarely cuneate, locules equal or subequal and extending the full length of the stamen connective, or unequal in length and raised from the stamen base on a short filament, stamen apex papillate or smooth, globose, umbonate, flattened or concave, or elongate and flattened, stamens all fertile or with outer whorl of staminodes or substaminodes. Carpels 1 to $\sim 50$, hairy, stigmas glabrous or hairy, elongate or flattened, lobes conical or U-shaped, ovules 2-30 in 2 lateral rows, or rarely 1, inserted apically or subapically. Fruit monocarps globose, oblong or cylindrical, stipitate or rarely sessile, flesh soft at maturity, pericarp smooth, warted, bearded or with longitudinal ridges, green, red or yellow in colour; Seeds 1-30, lateral in 2 rows, or rarely apical or subapical, testa brown, smooth, ruminations visible, aril circular or triangular.

\section{Key for flowering material}

Note: flowers of $U$. vietnamensis are unknown; this taxon is identified only in the fruiting specimen key.

1. Inner petals without conspicuous marginal glands, stamen locules equal or subequal in length, extending full length from connective apex to stamen base, carpels $10-50+$, stigma glabrous, ovules $2-30+$, laterally inserted in 2 rows ....................................... Uvaria zeylanica-group Inner petals often with conspicuous marginal glands at base, stamen locules unequal in length, raised on a short filament, extending $0.5-0.85$ the length from connective apex to stamen base, carpels $10-30$, or rarely 1 , stigma hairy or rarely glabrous, ovules $1-3$, apically or subapically inserted in 1 or 2 rows, or ovules $2-16$, laterally inserted in 2 rows. Uvaria hahnii-group

\section{Key to continental Asian species of the Uvaria zeylanica group}

1. Petals ovate, elliptical, oblong or obovate, acute or obtuse, spreading, often reflexed, membranous or fleshy, stigma short with broad U-shaped lobes

Petals broadly ovate, (bluntly) acute, erect, occasionally spreading but never reflexed, fleshy, never membranous, stigma elongate, conical or

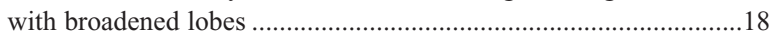

2. Calyx calyptrate in bud..................................................................... 3 Calyx valvate or, if fused, apex splitting early to leave connate (sometimes cup-shaped) ring around lower part of bud..................12

3. Pedicel and calyx warty with dense indument of many-branched thickly set hairs ......

Pedicel and calyx often hirsute, with uniform indument of ferruginous

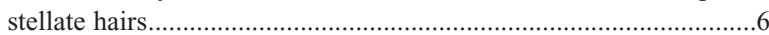

4. Leaf indument on lower surface ferruginous; compound pedunclepedicel length $<15 \mathrm{~mm}$; stipe of mature monocarp $<20 \mathrm{~mm}$ long...... .. 1. U. lamponga

Leaf indument on lower surface sparse to subglabrous; compound peduncle-pedicel length $>15 \mathrm{~mm}$; stipe of mature monocarp $>20 \mathrm{~mm}$ long..

5. Petals $\leq 6$, red throughout, obovate, apex obtuse, concave; apicule of mature monocarp rudimentary ..................................... U. concava

Petals $\geq 6$, red with yellow base, elliptical, apex bluntly acute, flattened; apicule of mature monocarp thick and prominent 5-10 $\mathrm{mm}$ long ...... 3. U. macropoda

6. Mature petals $20-50 \mathrm{~mm}$ long, stamens and carpels $\geq 6 \mathrm{~mm}$ long........ 4. U. grandiflora

Mature petals $<22 \mathrm{~mm}$ long, stamens and carpels $<6 \mathrm{~mm}$ long ...........7

7. Flowers $30-45 \mathrm{~mm}$ in diameter, buds $7.5-15 \mathrm{~mm}$ in diameter before opening

Flowers $<30 \mathrm{~mm}$ in diameter, buds $5-7.5 \mathrm{~mm}$ in diameter before

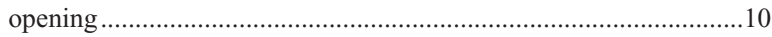

8. Young shoots, leaves, pedicel and outer surface of calyx with persistent ferruginous hairs $>0.5 \mathrm{~mm}$ long and 1-3-branched hairs...

5. U. curtisii

Young shoots, leaves, pedicel and outer surface of calyx with deciduous hairs $\leq 0.4 \mathrm{~mm}$ long and 1- to many-branched hairs, subtomentose, later sparsely hairy or subglabrous ..................................................

9. Leaf apex cuspidate or acuminate; bracts narrowly ovate-acute, $\sim 2 \mathrm{~mm}$ wide, often lost at maturity; calyx splitting unevenly into 2-4 sepals; fruit oblong, stipe $<15 \mathrm{~mm}$ long ........................................... U. dac

Leaf apex acute; bracts broadly obovate to circular-ovate, often wrapping around pedicel, $\sim 10 \mathrm{~mm}$ wide, persistent; calyx splitting evenly into 3 sepals; fruit globose to oblong, stipe $>15 \mathrm{~mm}$ long .......

7. U. hamiltonii

10. Pedicel $>20 \mathrm{~mm}$ long with prominent bracts ............... 8. U. macclurei Pedicel $<20 \mathrm{~mm}$ long, bracts not prominent .....................................11 
11. Petals yellow, oblong, apex obtuse, erect or partially recurved at maturity, never reflexed ........................................ 9 . U. calamistrata Petals red, elliptical, apex acute, fully reflexed at maturity

10. U. zeylanica

12. Calyx splitting apically in early bud to form 3-pointed connate ring around base of unopened corolla ..................................................13

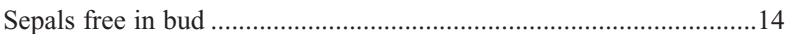

13. Leaves $70-200 \mathrm{~mm}$ long, base retuse or shortly cordate, veins $\leq 12$; inner petals opening with outer petals, never connivent

11. U. sphenocarpa

Leaves $160-450 \mathrm{~mm}$ long, base cordate, veins $\geq 12$; inner petals connivent, opening before senescence ............ 12. U. semecarpifolia

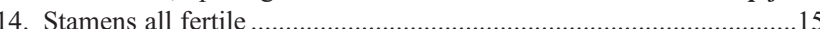
Outer whorl of staminodes or substaminodes present........................17

15. Corolla basally connate at maturity ............................... 13. U. narum

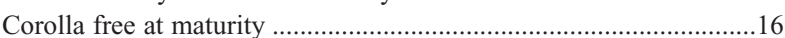

16. Leaves $>150 \times 100 \mathrm{~mm}$, veins $5-25$; inflorescence of $2-5$ buds in tightly packed rhipidium, up to 3 open at any time, pedicels broad and flattened with abaxial groove, bracts broadly obovate, clasping,..

..14. U. littoralis

Leaves $<150 \times 80 \mathrm{~mm}$; veins $9-12$; inflorescence of 1 or 2 , rarely 3 buds, pedicel not flattened not grooved, bracts elliptic-oblong, free

15. U. microcarpa

17. Leaves distinctly scabrid on upper surface ………............. 16. U. rufa Leaves often smooth and waxy, subglabrous to glabrous on upper surface

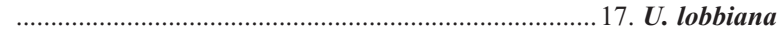

18. Peduncle elongate, $\sim 20-50 \mathrm{~mm}$ long ..........................19. U. griffithii Peduncle $<10 \mathrm{~mm}$ long, or pedicels sessile ........................................19

19. Pedicel elongate, $30-90 \mathrm{~mm}$ long ..................................................20 Pedicel $\leq 25 \mathrm{~mm}$ long ....................................................................22

20. Pedicel $>1.5 \mathrm{~mm}$ wide, flattened ............................ 20. U. tonkinensis Pedicel $<1.5 \mathrm{~mm}$ wide, not flattened .................................................21

21. Pedicel $35-40 \mathrm{~mm}$ long, $1 \mathrm{~mm}$ wide, outer petals $>6 \mathrm{~mm}$ long $\times>5 \mathrm{~mm}$ wide, inner petals $>6 \mathrm{~mm}$ long $\times>4 \mathrm{~mm}$ wide - north-eastern Vietnam and southern China ……....................................... 21. boniana

Pedicel $\sim 30 \mathrm{~mm}$ long, $<1 \mathrm{~mm}$ wide, outer petals $<6 \mathrm{~mm}$ long $\times<5 \mathrm{~mm}$ wide, inner petals $<6 \mathrm{~mm}$ long $\times<4 \mathrm{~mm}$ wide - central Vietnam.....

22. U. flexuosa

22. Leaves distinctly oblong, base subcordate-obtuse .......... 23. U. pierrei Leaves obovate, elliptical or lanceolate, base cuneate or retuse........23

23. Leaves elliptical, lanceolate or narrowly ovate, $70-150 \times 20-50 \mathrm{~mm}$

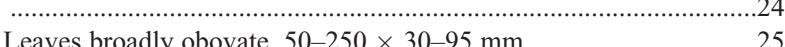

4. Leaf base cuneate, outer petals $<6 \mathrm{~mm}$ long $\times<5 \mathrm{~mm}$ wide, inner petals $\sim 3.35 \mathrm{~mm}$ long $\times<3 \mathrm{~mm}$ wide, stigma lobes broad - Myanmar, Indochina, Andamans, Malesia ................................ 24. U. micrantha

Leaf base subcordate, outer petals up to $>6 \mathrm{~mm}$ long $\times>5 \mathrm{~mm}$ wide, inner petals 5-8.5 mm long $\times>3 \mathrm{~mm}$ wide, stigma lobes narrow, elongate south-western Thailand, Taninthari, Myanmar.........25. U. utteridgei

25. Inflorescence from younger shoots, petals coriaceous, margin prominently tomentose, petals $<7 \mathrm{~mm}$ long, not spreading at maturity - western Indochina, Assam ......................26. U. argentea Inflorescence from mature shoots or older wood, petals fleshy, margin not tomentose, petals $>7 \mathrm{~mm}$ long, often spreading at maturity Isthmus of Kra to Sumatra 27. U. wrayi

\section{Key to continental Asian species of the Uvaria hahnii group}

1. Carpels 1 28. U. dasoclema Carpels $>1$ ....2

2. Ovules $1-3$, apically or subapically inserted.........................................

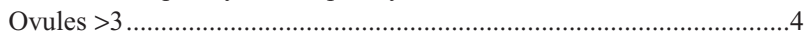

3. Habit an erect shrub, leaves broadly elliptic to oblong, base cordate, $120-150 \times 50-80 \mathrm{~mm}$, inner petals basally glabrous with conspicuous marginal glands, ovules 1 .
Habit a scandent shrub or woody climber, leaves ovate to elliptic to narrowly obovate, base shortly cordate or retuse, $95-140 \times 50-60 \mathrm{~mm}$, inner petals basally glabrous but without marginal glands, ovules 1-3

30. U. pauciovulata

4. Sepals ovate-lanceolate, leaf-like with prominent central vein, up to $\sim 10 \times$ $15 \mathrm{~mm}$

31. U. hahnii

Sepals ovate-acute, fleshy or coriaceous, prominent vein lacking, up to $\sim 3$ $\times 6 \mathrm{~mm}$

5. Inner petals with basal marginal glands, petals coriaceous to membranous, broadly elliptical-acute at first, expanding to obovate-acute at maturity, flattened, $\sim 21.5 \times 11.5 \mathrm{~mm}$, ovules $\sim 14-16 \ldots \ldots \ldots \ldots \ldots . . . .32 . \boldsymbol{U}$. dulcis

Inner petals without marginal glands, petals fleshy, remaining ovate and concave, $\sim 8 \times 8 \mathrm{~mm}$, ovules $\sim 4-8 \ldots \ldots \ldots \ldots \ldots \ldots \ldots \ldots . . . . .33$. U. siamensis

\section{Key for fruiting material of all taxa in continental Asia}

1. Shrub with shortened horizontal stems and erect branches; monocarps red, ellipsoidal, single seed with apical placentation .

29. U. ferruginea

Scandent shrub or climber with vertical stem, placentation lateral or subbasal, seeds 1 to many ..................................................................

2. Monocarps 10-30+, 1-4-seeded, globose, ovoid or transversely ovoid; pericarp thin; stipes typically long and narrow, never wider than $2 \mathrm{~mm}$ Monocarps 2-10(-20), 4-30-seeded, globose, ovoid or oblong; pericarp thickened, fleshy; stipes typically short and thickened or long with with deep striations

3. Fruit transversely ovoid with prominent apicule, densely tomentose;

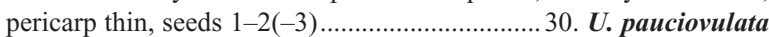
Monocarps largely glabrous, apicule when present small and blunt,

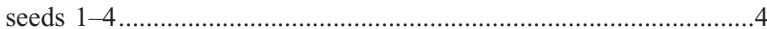

4. Leaves elliptic to broadly elliptic to shortly obovate, base retuse-shortly cordate, apex acute, veins $15-18$, subparallel, frequently branching basally and medially; indument tomentose on younger shoots, later sparse becoming subglabrous, except over midrib with tomentose $\sim 1 \mathrm{~mm}$ long erect hairs ................................................32. U. dulcis Leaves narrowly ovate to elliptic to obovate, base cuneate to retuse, apex bluntly acute to acuminate, usually waxy; veins (6-)8-14(-16), rarely branching basally or medially; indument occasionally persistent over abaxial midrib, elsewhere hairs sparse, usually glabrous or subglabrous, hairs less than $0.5 \mathrm{~mm}$ long........................................

5. Leaves $100-250 \mathrm{~mm}$ long, broadly elliptical to obovate, base broadly retuse to narrowly cordate, apex often with prominent acuminum...6 Leaves 50-150 mm long, narrowly ovate-elliptical-obovate or narrowly oblong, base cuneate or narrowly retuse, apex acute ........................7

6. Leaves elliptical-narrowly obovate, base retuse, apex acuminate or rarely acute, $\sim 130-170 \times 50-60 \mathrm{~mm}$, thinly coriaceous, waxy

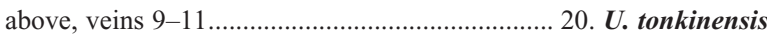
Leaves obovate, less often oblong, base retuse, apex acute-cuminate, up to $250 \times 95 \mathrm{~mm}$, membranous, veins $12-15 \ldots \ldots \ldots \ldots \ldots . .27$. U. wrayi

7. Leaves distinctively narrow-oblong, base retuse, apex obtuse or retuse, leaf margin turned downward ....................................... 23. U. pierrei Leaves ovate-elliptical, base cuneate, apex acute to narrowly acute, leaf

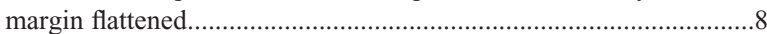

8. Monocarps 10-20, green turning yellow, globose to shortly oblong, $\sim 9.5 \times 8 \mathrm{~mm}$ at maturity, no apicule present; smooth; stipe 14-20(-23) mm, smooth, seeds $1-2(-3)$................24. U. micrantha Monocarps 25-45+, green turning red or purple at maturity, ovoid, to shortly cylindric, $\sim 15-17(-20) \times 11-13 \mathrm{~mm}$, surface often warty with short scattered stellate hairs; stipe $\sim 35-45 \mathrm{~mm}$ long, striate with sparse hairs, seeds 4-6 ..................................................... U. boniana

9. Monocarps sessile, in pseudo syncarpous conglomerate.

11. U. sphenocarpa

Monocarps stipitate, sides not flattened against adjacent monocarps 
10. Monocarp with dense covering of epidermal outgrowths...................11 Monocarp smooth, striate or tubercled, but without epidermal outgrowths.

11. Monocarps 20+, with dense beard of 3-6 mm long hair-like outgrowths, each carrying a tomentum of rufous stellate hairs; stipe up to $45 \mathrm{~mm}$ long $\times 2 \mathrm{~mm}$ in diameter... 18. $U$. vietnamensis Monocarps 2-12, initially covered with prominent beard of 5-8 mm long membraneous bluntly triangular outgrowths, later tuberculate with scattered remnants of outgrowths over surface; stipe short, $3-5 \mathrm{~mm} \times 2.5-3 \mathrm{~mm}$

9. U. calamistrata

12. Monocarp epidermis deeply grooved and warted; stipe $2-5 \mathrm{~mm}$ wide, 5-10 mm long, deeply striate...

Monocarp smooth, glabrous, or where tubercled stipe much greater than $10 \mathrm{~mm}$ long . .14

13. Leaves narrowly elliptic-obovate, acute-distinctly acuminate, base retuse, $\sim 180-190 \times 60-80 \mathrm{~mm}$; veins 11-13; monocarps 1-8(-10), $\sim 45 \times 25 \mathrm{~mm}$ in diameter, pericarp thickly striate; seeds $6-12 \ldots \ldots \ldots$.

6. U. dac

Leaves elliptical-narrowly obovate, apex acute, shortly acuminate or rarely obtuse, base cuneate-retuse, $\sim 110-160 \times 40-60 \mathrm{~mm}$, veins (9-) $10-12$, monocarps $1-4,40-45 \times 30-35 \mathrm{~mm}$ in diameter, pericarp tuberculate, seeds 6-10

8. U. macclurei

14. Stipe short, $\sim 2-4 \mathrm{~mm} \times 2 \mathrm{~mm}$; monocarps oblong, pale yellow or cream in colour at maturity.

Stipe $\sim 5-50 \times 1.5-4 \mathrm{~mm}$; monocarps green, red or purple at maturity, glabrous or hairy....

15. Leaves broadly elliptic to narrowly obovate 31. U. hahnii Leaves lanceolate or elliptical or ovate to elliptic to oblanceolate ....16

16. Leaves lanceolate or elliptical, base retuse, apex acute, $\sim 90-150 \times$

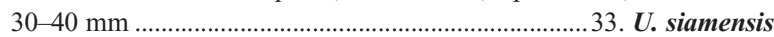

Leaves ovate-elliptic-oblanceolate, base cuneate, apex acuteacuminate, $\sim 55-70(-95) \times 20-28(-35) \mathrm{mm}$............ 10. U. zeylanica

17. Stipe elongate, $\sim 50-150 \times 2-3 \mathrm{~mm}$, striate....................................19

Stipe 5-50 mm long, smooth or shallowly striate..............................18

18. Monocarp globose or ovoid, $\sim 16-18 \times 20-25 \mathrm{~mm}$, prominently tubercled; seeds 4-6 ...............................................17. U. lobbiana

Monocarp oblong or cylindrical, $235-70 \times 20-30 \mathrm{~mm}$, smooth or somewhat tubercled; seeds $2-16$....................................................20

19. Monocarp narrowly cylindrical with prominent tapering apicule

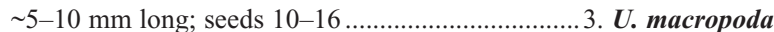

Monocarp shortly oblong to oblong, apicule when present $\sim 1-2 \mathrm{~mm}$ long; seeds 2-10..................................................... U. concava

20. Monocarp oblong, glabrous to subglabrous ......................................21

Monocarp broadly ellipsoidal to oblong, ferrugino-tomentose until

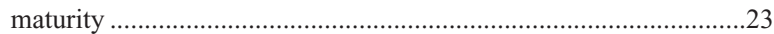

21. Stipe narrow, $\sim 10-35 \times 1-2 \mathrm{~mm}$ in diameter; monocarps $\sim 10-20 \times$ 6-10 mm

.....22

Stipe short, thickened, $2-10 \times 2-4 \mathrm{~mm}$ in diameter; monocarps up to $\sim 30 \times 16.5 \mathrm{~mm}$ 14. U. littoralis

22. Monocarps $4-8+$, green turning yellow then dark purple at maturity, ovoid to shortly oblongoid $\sim 20 \times 10 \mathrm{~mm}$ in diameter; stipe $10-15 \times$

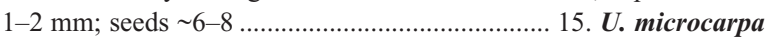
Monocarps 5-20+, green turning orange then red, globose to oblong, 10-15 $\times 6-8 \mathrm{~mm}$ in diameter; stipe $25-35 \times 1 \mathrm{~mm}$ in diameter; seeds $2-6$.

13. U. narum

23. Monocarps oblong-cylindrical, $50-75 \times 10-20 \mathrm{~mm}$ in diameter;

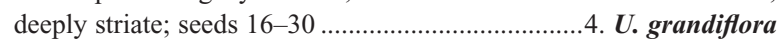

Monocarps globose-cylindrical, up to $\sim 30 \times 20 \mathrm{~mm}$ in diameter; smooth or tubercled, seeds 5-20+

24. Stipe $10-15 \mathrm{~mm}$ long; leaves broadly obovate $160-400 \mathrm{~mm}$ long, thickly coriaceous, glabrous above at maturity ..........12. U. semecarpifolia

Stipe 25-35 mm long; leaves obovate, 100-200 mm long, coriaceous, upper surfaces scabrous to sparse at maturity .....
25. Monocarps $10-14$, globose-oblong, $\sim 20 \times 15 \mathrm{~mm}$ in diameter, young? fruits green turning red; outer surface ferrugino-tomentose, indument tubercled; seeds 6-12.

7. U. hamiltonii

Monocarps 4-8(-20), cylindrical, 25-35 × 14-21 mm in diameter, young fruits green with rufous tint, turning bright red, outer surface subglabrous, seeds $5-20+$ 16. U. rufa

\section{Uvaria zeylanica group}

\section{Uvaria lamponga Scheff., Natuurk. Tijdschr. Ned.-Indië 31: 2 (1869)}

Type citation: 'in Sumatra, prov. Lampongs, speciem invenit TEYSMANN...'.

Type: $\mathrm{BO}$, n.v.

Uvaria andamanica King, J. Asiat. Soc. Bengal Pt. 2, Nat. Hist. 61(1): 21 (1892).

Type: INDIA. Andaman Islands, south Andaman, Port Blair, 1 Apr. 1884, King's Collector [H.H.Kunstler] 207 (lecto: CAL 000004186!, fide D.Mitra, Fasc. Fl. India 10: 14 (1982)).

[Uvaria ferruginea auct. non J.E.Teijsmann \& S.Binnendjik, Cat. Hort. Bot. Bogor. 175 (1866)].

Climber. Young shoots stout, subtomentose or floccose, becoming subglabrous and striate, bark black, hairs manybranched, rufous. Leaves narrowly obovate to oblanceolate, base retuse, apex acuminate, 150-250 × 60-80 mm, acumen up to $15 \mathrm{~mm}$, veins 13-23; indument variable, upper blade glabrous, sparse erect ferruginous hairs over midrib and primary veins, hairs on lower surface many-branched, ferruginous, tuberculate, becoming subtomentose to subglabrous. Petiole 5-6 $62 \mathrm{~mm}$, tomentose. Inflorescence leaf-opposed, flowers 2 or 3 , or up to 5 in a branching rhipidium, peduncle 2-5 mm long, pedicel 4-10 $\mathrm{mm}$ long, basal and medial bracts ovate, 4-5 $\times$ 4-5 $\mathrm{mm}$, peduncle, pedicel and bracts tuberculate, hairs as for lower surface of leaves. Calyx calyptrate in bud, sepals 3 , broadly ovate, $7-8 \times 4 \mathrm{~mm}$, basally connate, outer surface as for pedicel, inner surface hairs fine, combed. Corolla 2 subequal whorls of 3 petals, red in colour, coriaceous, broadly ovate, $\sim 10 \times 6 \mathrm{~mm}$, apices incurved. Stamens numerous, 3-4 $\mathrm{mm}$ long, locules running full length of stamen, apex flattened. Carpels numerous, 3-4 mm long, ovary tomentose, stigma glabrous, U-shaped, ovules 4-6. Fruit monocarps 5-30, ovoid to oblongoid, $\sim 18 \times 25 \mathrm{~mm}$, pericarp thick, epidermis warted, hairs many-branched, spreading, deciduous, stipe $\sim 10 \times$ 3-4 mm; seeds 1-6, compressed-ellipsoid, $\sim 12 \times 10 \times 5 \mathrm{~mm}$.

\section{Distribution}

Uvaria lamponga is a very rare species, with only isolated collections, all from mixed monsoon forest. The distribution is shown in Fig. 8.

\section{Notes}

Uvaria lamponga is similar to both $U$. concava Teijsm. \& Binn. and U. macropoda Hook.f. \& Thomson; however, the shorter stipe and floccose indument on the underside of the leaves should make it easily distinguishable. The indument is similar 


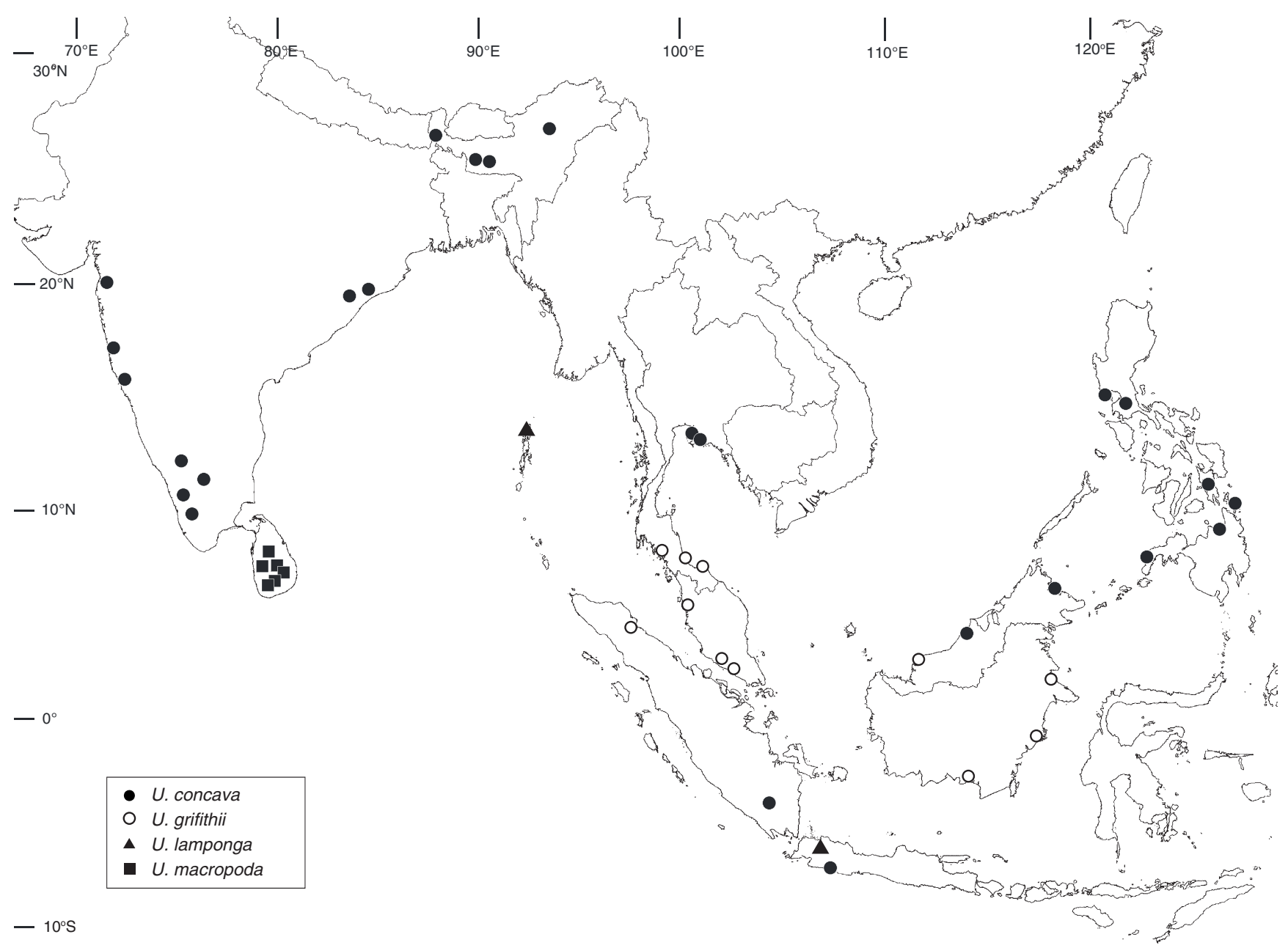

Fig. 8. Distribution of Uvaria concava, U. grifithii, U. lamponga and U. macropoda in southern and South-east Asia on the basis of herbarium specimens verified in this study.

to U. lobbiana Hook.f. \& Thomson, but this latter species has valvate sepals, an outer whorl of staminodes and a 35-70-mmlong stipe.

\section{Uvaria concava Teijsm. \& Binn., Natuurk. Tijdschr. Ned. Indië 3: 331 (1852)}

Type: INDONESIA. Java, cultivated in Hort. Bot. Bogor, s. dat., S.Binnendijk HB 17623 (lecto: BO 1824857 (image!), fide I.M.Turner, Phytotaxa 36: 97 (2011)).

Uvaria lurida Hook.f. \& Thomson, Fl. Ind. 1: 101 (1855).

Type: INDIA, Assam, Khasia, $2000 \mathrm{ft}$ [ 609.6 m], 2 Oct. 1850, J.D.Hooker \& T.Thomson 2468 (lecto: K 000380703!, fide I.M.Turner, Phytotaxa 36: 97 (2011)).

Uvaria narum var. macrophylla Hook.f. \& Thomson, Fl. Brit. India 1: 50 (1872); Uvaria hookeri King, Ann. Roy. Bot. Gard. (Calcutta) 4: 28, t. 22 (1893).

Type: INDIA. Kerala, Travancore mountains, Oct. 1814, Wall. Cat. no. 6473C (lecto: K-W 000442824!, fide I.M. Turner, Nordic J. Bot. 33: 289 (2015)).
Unona leytensis Elmer, Leafl. Philipp. Bot. 5: 1744 (1913); Uvaria leytensis (Elmer) Merr., Philipp. J. Sci., C 10(4): 230 (1915).

Type: PHILLIPINES. Mindanao, Province of Agusan, Mt Urdaneta, s. dat., A.D.E.Elmer 13880 (lecto: L 0190815!, fide I.M.Turner, Phytotaxa 36: 98 (2011)).

Uvaria eucincta Bedd. ex Dunn, Bull. Misc. Inform. Kew 1914: 182 (1914).

Type: INDIA. Madras, Russelondak Hills, s. dat., R.H. Beddome 50 (lecto: CAL 000004182!; isolecto: BM 000527912!, p.p., fide D.Mitra, Fasc. Fl. India 10: 15 (1982)).

Large woody climber up to $30 \mathrm{~m}$ tall. Young shoots tomentose, indument becoming sparse very quickly, hairs ferruginous, many-branched. Leaves elliptic-oblong to narrowly obovate, base retuse, apex acute or less often acuminate, $\sim 130-150 \times 40-50 \mathrm{~mm}$, coriaceous; veins $\sim 10-12$; indument upper surface entirely glabrous, lower surface indument sparse, hairs many-branched, blunt. Petiole 7-10 × $1.25 \mathrm{~mm}$, hairs sparse, blunt, many-branched, abaxial groove deep, often overtopped by margins, transverse foliations distinct, warty. Inflorescence terminal or leaf-opposed by apposition, flowers 
solitary or with dormant secondary bud, peduncle sometimes evident up to $5 \mathrm{~mm}$; pedicel $\sim 15-20(-50) \times 2.5 \mathrm{~mm}$, tuberculate, peduncle-pedicel often distinctly broader than supporting shoot, buds globose to conical with prominent apicule. Calyx calyptrate, sepals $(2-) 3(-4)$, basally connate, $\sim 11 \times 10 \mathrm{~mm}$, ovate-acute, concave, outer surface with same indument as pedicel, inner surface with subtomentose indument of fine, few-branched straggling hairs, subglabrous basally. Corolla of 6 (rarely 4) petals, basally connate in a single whorl, petals dark pink or red in colour, very broadly obovate, apex obtuse, $\sim 20 \times$ $15 \mathrm{~mm}$, outer surface densely tomentose with many-branched pale to rufous hairs, less tuberculate than calyx and pedicel, inner surface subtomentose as for inner surface of calyx, but denser, petals detaching from receptacle as connate ring. Stamens numerous, $\sim 3.5 \mathrm{~mm}$ long, glabrous, locules extending to full length of stamen, connective apex flattened or shallowly truncate. Carpels numerous, $\sim 3.5 \mathrm{~mm}$ long, ovary with flattened, manybranched spreading hairs, stigma short, U-shaped, not distinctly lobed, ovules $8-10$ in two rows. Fruit monocarps $\sim 5$ to many, green turning orange or yellow at maturity, smooth, globular-oblongoidal, $\sim 15-30 \times 10-17 \mathrm{~mm}$, often with 1 or more longitudinal ridges, small apicule often present, occasional constrictions between seeds, pericarp soft, fleshy at maturity, stipe long, 50-100(-120) × 2-4 mm, typically flattened, often with prominent longitudinal ridges or striations, young stipe and monocarp with sparse indument, later subglabrous; seeds $~ 2-10$ in two closely packed rows, ovoid-compressed ovoid, 8-11 $\times 6-9$ $\times 3-5 \mathrm{~mm}$, smooth, aril and raphe not prominent. (Fig. 1F.)

\section{Distribution}

This is the most widely distributed Uvaria species in Asia, covering almost the entire distribution of the genus. The distribution is shown in Fig. 8.

\section{Notes}

This taxon is widely distributed, with much continuous variation in floral and fruit characters. Even though Zhou et al. (2012) identified some variation at molecular level between Indian (identified as U. lurida Hook.f. \& Thomson) and Indonesian (identified as $U$. concava) accessions, morphometric analysis by Meade (2001) demonstrated that $U$. concava, $U$. eucincta Bedd. ex Dunn and $U$. lurida differ only in continuous characters. King elevated U. narum var. macrophylla of Hooker and Thomson to $U$. hookeri. Both accounts distinguished this taxon by the co-occurrence of long, thick stipes and small, monocarps with seeds in two rows; however, the specimens in question are merely young fruiting specimens of $U$. concava (Meade 2001). The most variable characters in $U$. concava are fruit size and pedicel length. Plants from wetter habitats produce typically larger fruits than those from elsewhere, and some of the Philippine specimens exhibit much longer pedicels than normal. However, the tuberculate inflorescence, unevenly splitting calyx and connate corolla are distinctive characters throughout the range, and none of the variation is distinctive or consistent enough to warrant the formal designation of varieties. The thickened stipe and larger monocarps of $U$. concava should make it easy to distinguish this species from U. narum (Dunal) Blume.
3. Uvaria macropoda Hook.f. \& Thomson, Fl. Ind. 1: 101 (1855)

Type: SRI LANKA. s. loc, s. dat, $1600 \mathrm{ft}[\sim 487.7 \mathrm{~m}]$, G.W. Walker 160 (lecto: K 000380706!, fide I.M.Turner, Nordic J. Bot. 33: 292 (2015)).

Climber up to $35 \mathrm{~m}$ tall. Young shoots subtomentose with blunt rufous hairs, quickly becoming deeply striate and grey in colour with prominent lenticels. Leaves elliptical-obovate, base narrowly retuse to cuneate, apex obtuse to shortly acute to acuminate, $80-130(-150) \times 30-50(-60) \mathrm{mm}$, coriaceous, waxy; veins $\sim 8-12(-14)$; indument upper blade completely glabrous, lower blade and midrib subglabrous with very rare rufous $\sim 10+$-branched hairs. Petiole $\sim 5-6(-8) \times 1 \mathrm{~mm}$, indument sparse, hairs blunt. Infloresence terminal or (sub)opposed by apposition, flowers solitary, pedicel $\sim 15-20 \times 1-1.5(-2) \mathrm{mm}$ in diameter, peduncle-pedicel distinctly broader than young shoots, narrowing at base, bracts usually wanting, buds spherical or conical with a prominent apicule, pedicel and bud covered with thickened, many-branched hairs, giving coarsly tuberculate appearance. Calyx calyptrate, splitting irregularly into $(2-) 3(-4)$ unevenly sized sepals, up to $\sim 10 \times 9 \mathrm{~mm}$, ovate, acute, often one larger sepal carrying apicule, outer surface indument as for pedicel, inner surface hairs smaller and finer, surface smooth, not tuberculate. Corolla of 6-8 petals, basally connate in a single whorl, petal bases fused for $1-4 \mathrm{~mm}$, petals red with yellow base, elliptical to oblong, acute, $\sim 18 \times 8 \mathrm{~mm}$, petal apices often concave but not distincly obovate, indument on outer surface densely tomentose with pale, many-branched fine hairs, overlain by scattered large rufous hairs as found on calyx and pedicel, more dense along central axis and towards base, especially on outer whorl, inner surface densely and shortly tomentose with no large rufous hairs. Stamens numerous, 2.25-3 mm long, glabrous, locules extending to full length of stamen, connective apex broad, convex or flattened or rarely shallowly truncate, shortly papillate. Carpels $\sim 30$, 3-3.75 mm long, ovaries tomentose with flattened spreading hairs, stigmas U-shaped with very large, broad lobes $\sim 1-1.25 \mathrm{~mm}$ wide, raised distinctly above stamens, some occlusion of stigma on external side, ovules 12-16. Fruit monocarps, $\sim 10-16$, narrowly cylindrical and angular with 4 distinct longitudinal ridges, $50-70 \times 10-15 \mathrm{~mm}$, apicule usually thickened and prominent, $\sim 5-10 \mathrm{~mm}$ long, pericarp thick but outline of seeds usually visible, indument sparse, stipes distinctly elongate, 65-150 $\mathrm{mm}+$, thickened at base and apex, deeply striate; seeds $10-16$, in two rows closely packed together to seem like one row, oval, $7 \times 5 \times 4 \mathrm{~mm}$, smooth, aril and raphe not well developed.

\section{Distribution}

Widely distributed in dry and moist areas of Sri Lanka, occasional in disturbed forest of the wetter south-western hills, absent from the southern and south-western lowlands. Distribution is shown in Fig. 8.

\section{Notes}

This taxon is intermediate in morphology between $U$. concava and $U$. narum, but easily distinguished from these two species by its few carpels with very large, broad stigmas and its 
distinctive fruits with a large, tapering apicule. Huber (1985) reported that in Ceylon U. narum and U. macropoda are ecologically distinct, with $U$. narum being restricted to the wetter south and south-west of the island.

\section{Uvaria grandiflora Roxb. ex Hornem., Hort. Bot. Hafn., suppl. 140 (1819)}

Type: INDIA. Bengal. Cultivated in the Calcutta Botanic Garden, Apr. 1818, Anon. s.n. (lecto: C 10005704!, fide I.M. Turner, Phytotaxa 36: 99 (2011)).

Uvaria grandiflora Roxb., Hort. Bengal. 43 (1814), nom. inval., nom. nud. Unona grandiflora Lesch. ex DC., Prodr. 1: 90 (1824).

Type citation: '(Leschenault! in litt. ... in Bengalo.' Type: ?P, n.v.

Uvaria purpurea Blume, Bijdr. Fl. Ned. Ind. 1: 11 (1825).

Type: INDONESIA. Java, s. dat., Blume s.n. (neo, here designated: NY 00059989!).

Uvaria grandiflora Roxb., Fl. Ind. ed. 1832 2: 665 (1832), nom. illeg., non Roxb. ex Hornem. (1819).

Type: INDIA. India Orientalis, s. dat., Wallich s.n. (neo, here designated: BM 000528031!).

Uvaria platypetala Champ. ex Benth., Hooker's J. Bot. Kew Gard. Misc. 3: 257 (1851).

Type: CHINA. Hong Kong, 1850, Champion 38 (lecto, here designated: K 000380694!).

Uvaria rhodantha Hance ex Walp., Ann. Bot. Syst. 2: 19 (1851).

Type: CHINA. Hong Kong, Apr. 1852, H.F.Hance 933 (neo: BM 000527943!, fide I.M.Turner, Phytotaxa 36: 99 (2011)).

Uvaria purpurea var. tuberculata King, J. Asiat. Soc. Bengal Pt. 2, Nat. Hist. 61: 18 (1892).

Type: MALAYSIA. Perak, Larut, Aug. 1883, King's Collector [H.H.Kunstler] 4786 (lecto: K 000380129!; isolecto: G!, fide J.Sinclair, Gard. Bull. Singapore 14: 203 (1955)).

Uvaria rubra C.B.Rob., Bull. Torrey Bot. Club 35: 68 (1908).

Type: PHILLIPINES. Santa Cruz, Davao Province, Mindanao, 29 June 1905, Williams 3042 (holo: NY 0005991!, iso: NY 0005992-3!).

\section{Notes}

The more commonly used name U. purpurea Blume (1825) postdates Horneman's publication of the original Roxburgh description (1819) of this taxon as $U$. grandiflora. In the case of both $U$. purpurea, and Roxburgh's later publication of the illegitimate later homonym U. grandiflora in 1832 (Roxburgh 1832), the original type material is lost, and the named neotypes (from the Blume and Wallich herbaria) are the only known material to have survived from the original collections, which Blume and Roxburgh reviewed in preparation of their taxonomic accounts. The fruit of $U$. grandiflora is variable. The monocarp can be wholly tubular or constricted between the seeds and the surface can be either smooth, striate or tuberculate. As the fruit matures, the pericarp softens, causing the outline of seeds within the monocarp to become more sharply defined. Uvaria grandiflora produces the largest bracts, sepals, petals, stamens and carpels of any Uvaria species in Asia, and the fruit also produces the most seeds per carpel (up to 30). Where the inflorescence produces more than one flower, the dimensions of all organs are reduced, and this can lead to confusion with large-flowered specimens of U. microcarpa Champ. ex Benth. However, U. microcarpa has an outer whorl of staminodes. Infertile specimens are most likely to be confused with $U$. littoralis, U. microcarpa and $U$. dac Pierre ex Finet \& Gagnep. The subcordate leaf base of $U$. grandiflora is usually enough to distinguish it from the two former species (which have cordate bases) and $U$. grandiflora also retains its leaf indument to a much greater extent than do these two species. The leaves of $U$. dac are smaller, have fewer veins and are much more acuminate than than those of $U$. grandiflora. The only variety recognised in continental Asia is var. flava, which is restricted to the wetter habitats of south-eastern and peninsular Thailand and produces cauliflorous inflorescences. The fruits of $U$. grandiflora are edible, but not as highly regarded as those of $U$. rufa Blume. The year-round production of large, fragrant flowers makes it a popular feature of tropical Asian botanic gardens. In Hainan, the leaves are used in the making of cakes and rice whisky.

\section{Key to the varieties in continental Asia}

1. Petals red, coriaceous, elliptical or oblong, $\sim 30-50 \times 12-20 \mathrm{~mm}-\mathrm{Sri}$ Lanka, Myanamar, Southern China, South-east Asia, Malesia ........... 4a. U. grandiflora var. grandiflora Petals red or white, fleshy, broadly ovate-elliptical, $20-40 \times 15-25 \mathrm{~mm}$ - south-eastern Thailand, Malay Peninsula, West Malesia 4b. U. grandiflora var. flava

4a. Uvaria grandiflora Roxb. ex Hornem var. grandiflora

Scrambling shrub up to $3 \mathrm{~m}$ or large woody climber up to $30 \mathrm{~m}$. Young shoots densely tomentose, becoming striate and subglabrous, flexuous. Leaves elliptic-obovate, base subcordate or very shortly retuse, apex obtuse-acute, 130-190(-250) $\times$ 50-75(-100) mm, subcoriaceous to coriaceous; veins 11-14 $(-16)$; indument upper surface sparse to subglabrous, hairs 1to many-branched, lower blade tomentose to rarely sparse, hairs all stellate, $\sim 10$-branched, $0.3-0.5 \mathrm{~mm}$ in diameter. Petiole short, 5-6 $\times 2 \mathrm{~mm}$, sparsely tomentose, warted and transversely foliated, black. Inflorescence leaf-opposed or subopposed, solitary or rarely with second dormant bud, or cauliflorous with elongate, often many-branched cyme of 3 to many pedicels, 1 or 2 bearing mature flowers, pedicel $10-15 \times$ $2 \mathrm{~mm}$, or up to $60 \times 2 \mathrm{~mm}$, ferrugino-tomentose, basally and medially bracteate, bracts large, ovate-elliptical, 10-33 $\times$ 5-20 mm, leafy, veins prominent, bud $15-25 \mathrm{~mm}$ in diameter before opening, indument densely tomentose to tomentose. Calyx calyptrate in bud, later forming (2-)3(-4) ovate-acute, 15-25 $\times$ 9-18 $\mathrm{mm}$ sepals, indument densely ferruginous, tomentose, short and paler on inside, longer on outside, sepals conspicuous, green, reflexing at maturity. Corolla 2 subequal whorls of 3 petals, petals deep red to purple, elliptical-oblong to rarely very narrowly obovate, $20-45 \times 8-15 \mathrm{~mm}$, apex obtuse or very bluntly acute, sparse stellate indument on inner surface of both whorls, more dense on outer surface. Stamens numerous, $6-8 \times 1.25 \mathrm{~mm}$, connective head shortly papillate, 
flattened, dorsal ridge often prominent, with stellate indument running length of connective, locules extending to full length of stamen. Carpels numerous, $8.0-8.5 \times 1.5 \mathrm{~mm}$, ovary shortly and densely tomentose, stigma U-shaped, glabrous, somewhat lobed, $2 \times 1-2 \mathrm{~mm}$ wide, producing copious secretions, ovules 24-36. Fruit monocarps 6-12(-20), receptacle up to $\sim 25 \mathrm{~mm}$ in diameter, stamen abscission scars forming prominent ring at base, stipes up to $30 \times 4 \mathrm{~mm}$, thickened towards base and apex, smooth to (thickly) striate, shortly and densely tomentose; monocarps 50-75 $\times 10-20 \mathrm{~mm}$, cylindrical, apex globose to conical, smooth or with longitudinal or lateral ridges and constrictions between seeds; seeds 16-30, hemi-ellipsoidal to ovoid, often dorso-ventrally flattened, up to $7 \times 5 \times 2 \mathrm{~mm}$, smooth, ruminations visible. (Fig. 1A.)

\section{Distribution}

Uvaria grandiflora var. grandiflora is found mostly in evergreen forest, but there are occasional records from semi-deciduous dipterocarp forest. Collected most frequently in Hongkong, Hainan and south-eastern Thailand and also in the middle and lower Malay Peninsula. Several early collections from
Tenasserim in Myanmar suggest that it is a common species there too; however, no recent material is available from this region. This species is most probably introduced in Sri Lanka. Uvaria grandiflora var. grandiflora also occurs in Malesia as far east as the Phillipines and New Guinea. Distribution is shown in Fig. 9.

4b. Uvaria grandiflora var. flava (Teijsm. \& Binn.) J.Sinclair, Gard. Bull. Str. Settlem. 3: 44 (1953)

Uvaria flava Teijsm. \& Binn., Natuurk. Tijdschr. Ned.-Indië 25: 419 (1863).

Type: INDONESIA. Bankga, cultivated Hort. Bog. XVI-E-6, s. dat., leg. ign., (lecto, here designated: L 1770656!).

Uvaria purpurea var. alba Scheff. Natuurk. Tijdschr. Ned.-Indië 31: 4 (1869).

Type: INDONESIA. Sumatra, Priaman, s. dat., Diepenhorst s.n. (lecto, here designated: U 1074870!).

Differs from the typical form of the species in having thick, fleshy, usually broadly and bluntly ovate petals, which can be either red or yellowish white in colour.

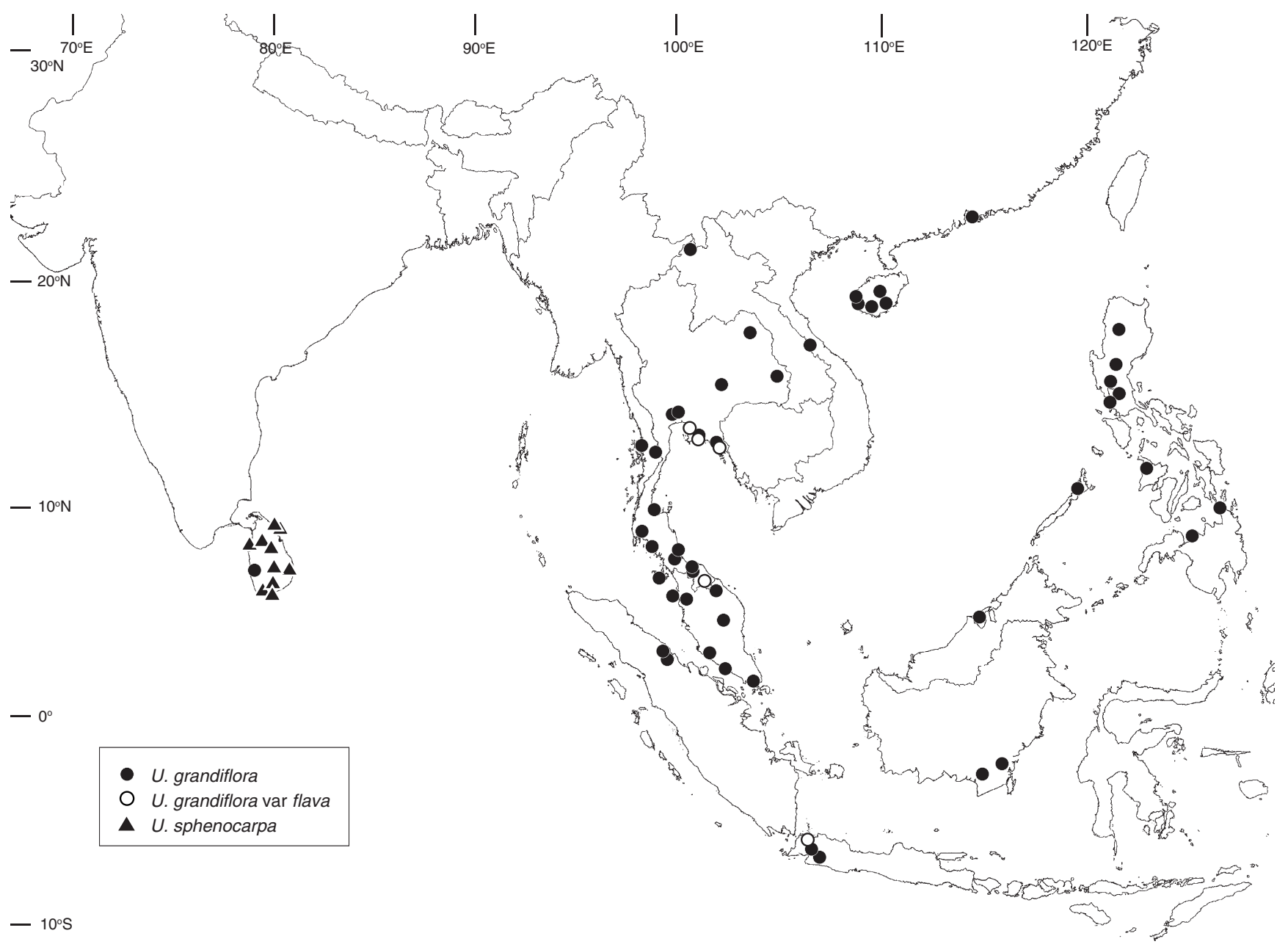

Fig. 9. Distribution of Uvaria grandiflora, U. grandiflora var. flava and U. sphenocarpa in southern and South-east Asia on the basis of herbarium specimens verified in this study. 


\section{Distribution}

Uvaria grandiflora var. Alava is recorded from evergreen forest sites in south-eastern Thailand, lower peninsular Thailand and western Java. Distribution is shown in Fig. 9.

\section{Uvaria curtisii King, J. Asiat. Soc. Bengal, Pt. 2, Nat. Hist. 61(1): 19 (1892)}

Type: MALAYSIA. Penang, Government Hill, $2000 \mathrm{ft}$ [ 609.6 m], May 1893, Curtis 1415 (lecto, here designated: S 0073636!; isolecto: BM 000527922!, G 00226139!, K 000380126-8!); MALAYSIA. Perak, Ulu Bubong, s. dat., King's Collector [H.H.Kunstler] 8543 (syn: C!).

Shrub or large climber up to $20 \mathrm{~m}$ tall, with distinctive hirsute indument. Young branchlets subtomentose, hairs ferruginous, hirsute, becoming sparse with age. Leaves subcoriaceous, elliptical to narrowly obovate, $\sim 210-250 \times 60-70 \mathrm{~mm}$, acute or shortly acuminate, base retuse; veins $10-12+$; indument hairs ferruginous, hirsute, $\sim 0.5-1 \mathrm{~mm}$ long, prominent at margin generating ferruginous tint. Petiole $\sim 8 \times 3 \mathrm{~m}$, ferrugino-tomentose, with long stellate hairs $0.6-1.0 \mathrm{~mm}$ long. Inflorescence leaf-opposed, flowers 2 , secondary bud normally dormant, peduncle $1.5-12 \mathrm{~mm}$, pedicels long and thin, up to $\sim 20-30 \times 1 \mathrm{~mm}$, basal bract narrowly elliptical, $\sim 8 \times 2 \mathrm{~mm}$, often clasping, medial bract ovate to broadly elliptical, $\sim 4-5 \times 2.5-3 \mathrm{~mm}$., bud $8-15 \mathrm{~mm}$ in diameter before opening, indument ferrugino-hirsute on peduncle, pedicel, bracts and early bud. Calyx calyptrate in bud, sepals $3, \sim 10 \times 6 \mathrm{~mm}$, ovate, acute to narrowly acute, apices sometimes reflexed at maturity, inner surface hairs fine, pale, subtomentose. Corolla 2 subequal whorls of 3 petals, petals white or greenish-yellow, elliptic-acute, $\sim 13 \times 6.5 \mathrm{~mm}$, membranous, spreading, hairs subtomentose, short, and pale on both surfaces, with scattered ferruginous hairs on outer surface, inner surface carrying prominent indents from stamen connectives. Stamens numerous, $3 \mathrm{~mm}$ long, glabrous, connectives papillate, truncate or somewhat flat and elongate in marginal stamens, ridge sometimes present on outer surface, locules extending to full length of stamen. Carpels numerous, $3 \mathrm{~mm}$ long, ovary with dense combed-tomentose indument, stigma U-shaped, lobes not broadened, ovules $\sim 20$ in 2 lateral rows. Fruit monocarps $10-30$, cylindrical or oblongoidal, $\sim 65 \times 25 \mathrm{~mm}$, tomentose, stipe $10-40 \times 2-3 \mathrm{~mm}$; seeds $\sim 16$, flattened ellipsoidal.

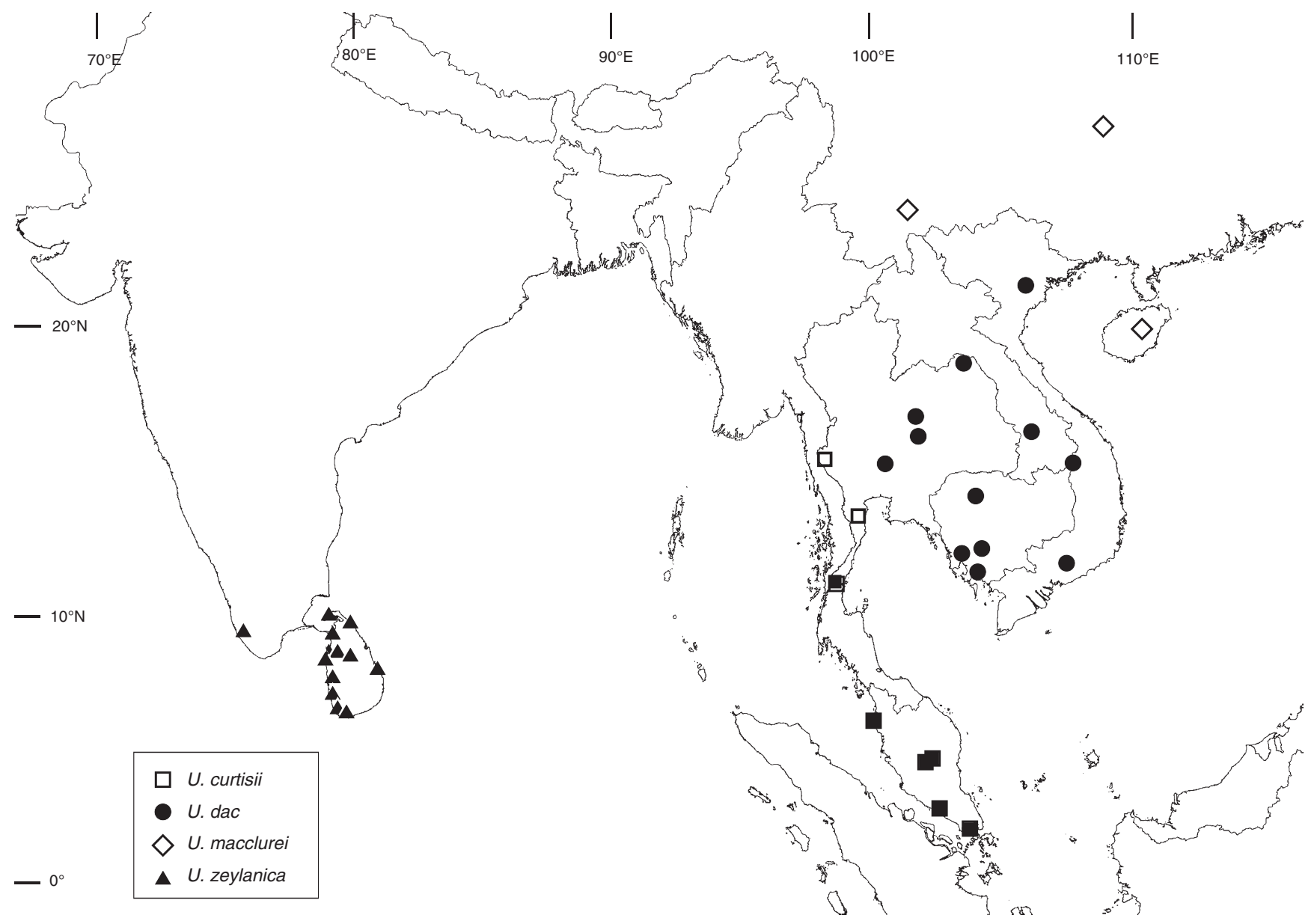

Fig. 10. Distribution of Uvaria curtisii, U. dac, U. macclurei and U. zeylanica in southern and South-east Asia on the basis of herbarium specimens verified in this study. 


\section{Distribution}

Newly recorded for Thailand. Distribution is shown in Fig. 10.

\section{Notes}

Morphologically similar to $U$. hirsuta Jack (lower Malay Peninsula), but distinguished from that species by its smaller yellowish-white petals ( $v$. the red petals of $U$. hirsuta), its shorter, less hirsute indument and its peculiar leaves, which have distinctly fewer, more narrowly angled veins than those of $U$. hirsuta (Meade and Parnell 2002). The lectotype selected presents the best-preserved material from the originally cited sheets.

\section{Uvaria dac Pierre ex Finet \& Gagnep., Bull. Soc. Bot.} France (Mém. 4 (2)) 53: 65 (1906)

Type: CAMBODIA. Samrong-tong, Cherreo Mountains, Apr. 1870, Pierre 748, (lecto, here designated: K 000582098!; isolecto: P 00262204-5!); CAMBODIA. Tpong, Tamir Mountains, May 1870, Pierre 748A (syn: K 000582096!, P 00262203!, P 00432438!); Kampot, Kam-Chay Mountains, May 1874, Pierre 1459 (syn: A!, PHH!, K 000582097!, P 00262206-8!).

Uvaria varaigneana Pierre ex Finet \& Gagnep., Bull. Soc. Bot. France (Mém. 4 (2)) 53: 64 (1906).

Type: VIETNAM. Bien Hoa, near Chiao-shan, 1875-77, Pierre 1785 (lecto, here designated: P 00262209!; isolecto: P 00262210-11!).

Climber or sarmentose shrub. Young branchlets ferruginotomentose, hairs quickly becoming blunt and sparse, bark striate. Leaves narrowly elliptic-obovate, acute-distinctly acuminate or cuspidate, base retuse, $\sim 180-190 \times 60-80 \mathrm{~mm}$; veins 11-13; indument upper-surface hairs 1-4-branched, lower-surface hairs 10-branched. Petiole $\sim 5-7 \times 2 \mathrm{~mm}$, tomentose becoming sparse. Inflorescence leaf-opposed, flowers solitary or rarely 2 or 3 , pedicel $8-15(-25) \mathrm{mm}$, often flattened to $\sim 2 \mathrm{~mm}$ broad, otherwise $\sim 1.25 \mathrm{~mm}$, bracts ovate-acute, $\sim 4-7(-10) \times 1.25-2 \mathrm{~mm}$, often wanting, bud $8-15 \mathrm{~mm}$ in diameter before opening, pedicel and bract densely ferruginotomentose. Calyx calyptrate in bud, splitting into 2-4 often unevenly sized ovate-acute sepals, each $(7-) 10 \times(8-) 10 \mathrm{~mm}$, at length spreading, outer indument tomentose to sparsely tomentose, ferruginous, inner surface subtomentose, hairs pale. Corolla 2 subequal whorls of 3 petals, petals yellow or rarely red, elliptic, obtuse-acute, spreading, $\sim 15-20 \times 8-10 \mathrm{~mm}$, inner and outer surfaces sparsely tomentose, whorls subequal. Stamens numerous, $\sim 4.5 \mathrm{~mm}$ long, glabrous, connective truncate, adaxial ridge often present, locules extending to full length of stamen, staminodes not present. Carpels $\sim 10-15, \sim 3.5 \mathrm{~mm}$ long, ovary densely ferrugino-tomentose, stout in appearance, apex narrowed into neck, stigma glabrous with conspicuously broad U-shaped lobes, partly occluded by extension of ovary wall above neck, ovules $\sim 16$ in 2 lateral rows. Fruit monocarps $1-8(-10)$, subbasally attached, $\sim 45 \times 25 \mathrm{~mm}$ in diameter, oblongoid, pericarp thickened, epidermis warted with fine tomentose indument, mature fruits green with brown tint, stipe 5-10 $\times 2.5-3 \mathrm{~mm}$, thickly striate, tomentose; seeds $6-12$ in 2 rows, $\sim 10 \times 6 \times 4 \mathrm{~mm}$, hemi-ellipsoidal.

\section{Distribution}

The distribution is shown in Fig. 10.
Notes

Uvaria varaigneana and $U$. dac were described by Finet and Gagnepain in the same work, based on Pierre's unpublished concepts. However, it and $U$. dac are merely flowering and fruiting specimens respectively of the same species. Uvaria $d a c$ is the name in more common usage, and is adopted here in preference to $U$. varaigneana. The species shows little phenotypic variation, save the occasional occurrence of red rather than yellow petals, and the presence of persistently tomentose leaves in some specimens from central Vietnam. The fruit is unusual in the genus, retaining a green colour up to maturity. The lectotype selected presents the best-preserved material from the originally cited sheets.

\section{Uvaria hamiltonii Hook.f. \& Thomson, Fl. ind. 1:96(1855)}

Type: INDIA. Bihar, Monghir or Goyalpara, Assam, 1808 or 1811, F.Buchanan-Hamilton s.n., Wallich Cat. no. 6485E (lecto: CAL; isolecto: BM 000949957!, K-W 001124004-5!, fide D.Mitra, Fasc. Fl. India 10: 16 (1982)).

\section{Notes}

Although both varieties of Uvaria hamiltonii share characters with many Uvaria species, there are a few characters by which they can readily be identified, namely (1) the two or three thick, elongate pedicels, (2) the basally free, obovate, concave petals and (3) the partially verrucose ferruginous indument present on the pedicel and calyx and the stipe and monocarp of ripening fruits. The distinctive leaf venation shared by both varieties also helps their identification from sterile specimens. The petals of $U$. hamiltonii var. kurzii can be similar to those of $U$. $d a c$, and, in specimens that lack fruits, the verrucose calyx and larger, more tomentose leaves of $U$. hamiltonii var. kurzii should make it possible to distinguish the two.

\section{Key to the varieties in continental Asia}

1a. Leaf veins $\geq 14$; petals red, distinctly obovate and concave, apex obtuse, 15-20 × 9-11 mm - Assam, Myanmar, northern Indochina, Hong Kong ............................................ 7a. U. hamiltonii var. hamiltonii

Leaf veins $<14$; petals white or yellow, elliptical to oblong, apex obtuse or rarely concave, 10-15 × 8-10 mm - Andaman Islands, Indochina, southern China 7b. U. hamiltonii var. kurzii

\section{7a. Uvaria hamiltonii Hook.f. \& Thomson var. hamiltonii}

Large climber up to $30 \mathrm{~m}$, or a scrambling, rarely upright, shrub. Young branchlets ferrugino-hirsute, becoming subglabrous and distinctly striate. Leaves (elliptic-)obovate, base subcordate to retuse, apex acute-acuminate, 115-175(-205) $\times$ 60-85 mm, membranous; veins $14-25$, sometimes branching basally or medially; indument upper blade subtomentose with simple and stellate hairs, lower surface hairs stellate only. Petiole short, $\sim 4 \times 2 \mathrm{~mm}$, not much thicker than midrib, warty, tomentose to subtomentose. Inflorescence flowers 1 or 2 or rarely 3, leaf-opposed or subopposed, peduncle $\sim 5 \mathrm{~mm}$, pedicel $\sim 35 \times 2 \mathrm{~mm}$, bracts broadly obovate to circular-ovate, often wrapping around pedicel, $\sim 10 \times 10 \mathrm{~mm}$, veins prominent, bud $8-15 \mathrm{~mm}$ in diameter before opening, hairs on peduncle, pedicel and bracts shortly ferruginous and tomentose, often verrucose. Calyx calyptrate in bud, sepals 3 
or 4, spreading, partially recurved, connate at base, triangular to very broadly ovate, bluntly acute, $7 \times 10 \mathrm{~mm}$, tomentose or verrucose on outer surface. Corolla 2 subequal whorls of 3 petals, petals red, subequal, distinctly obovate, apex broadly concave-rounded, $\sim 10-22 \times 10-14 \mathrm{~mm}$, densely and shortly tomentose on both surfaces. Stamens numerous, 2.5-3 mm long, connective apex short, papillae often prominent, locules rarely marginally unequal in length. Carpels numerous, $\sim 2 \mathrm{~mm}$ long, ovary densely tomentose, stigma glabrous, U-shaped, ovules 10-12. Fruits monocarps $\sim 10-14$, globose to oblong, $\sim 20 \times$ $15 \mathrm{~mm}$, green turning red, pericarp fleshy or thin, flesh pale white in colour, outer surface ferrugino- tomentose, often verricose, stipe $\sim 25-35 \times 1.25-2.25 \mathrm{~mm}$, striate, especially when thick, tomentose, monocarp attachment basal or sub-basal; seeds 6-12, $\sim 11 \times 4 \times 2 \mathrm{~mm}$, ellipsoidal, laterally compressed, smooth, brown, ruminate.

\section{Distribution}

Uvaria hamiltonii var. hamiltonii has a northerly distribution relative to the remainding species in the genus. Plants originally from Assam have been cultivated since the early 1800s at Peradeniya Gardens in Sri Lanka (where it has become naturalised) and also at Bogor Gardens in Java. Like most Uvaria species in mountain or disturbed habitats, the species is often found in gullies and along watercourses where the soil is deep and damp. At the northernmost latitudes, $U$. hamiltonii var. hamiltonii has not been collected above $650 \mathrm{~m}$, and so is essentially a lowland tropical species. Distribution is shown in Fig. 11.

7b. Uvaria hamiltonii var. kurzii King, J. Asiat. Soc. Bengal, Pt. 2, Nat. Hist. 61(1): 14 (1892)

Uvaria kurzii (King) P.T.Li, Acta Phytotax. Sin. 14(1): 106 (1976).

Type: INDIA. Andaman Islands, near Port Blair, 9 July 1884, King's Collector [H.H.Kunstler] 338 (lecto: CAL 000004190 (image!), fide D.Mitra, Fasc. Fl. India 10: 16 (1982)).

Differs from the typical form of the species in having smaller leaves with 10-13 subparallel veins and a white to yellow corolla with coriaceous, elliptical to oblong, obtuse, $10-15 \times 8-10 \mathrm{~mm}$ petals.

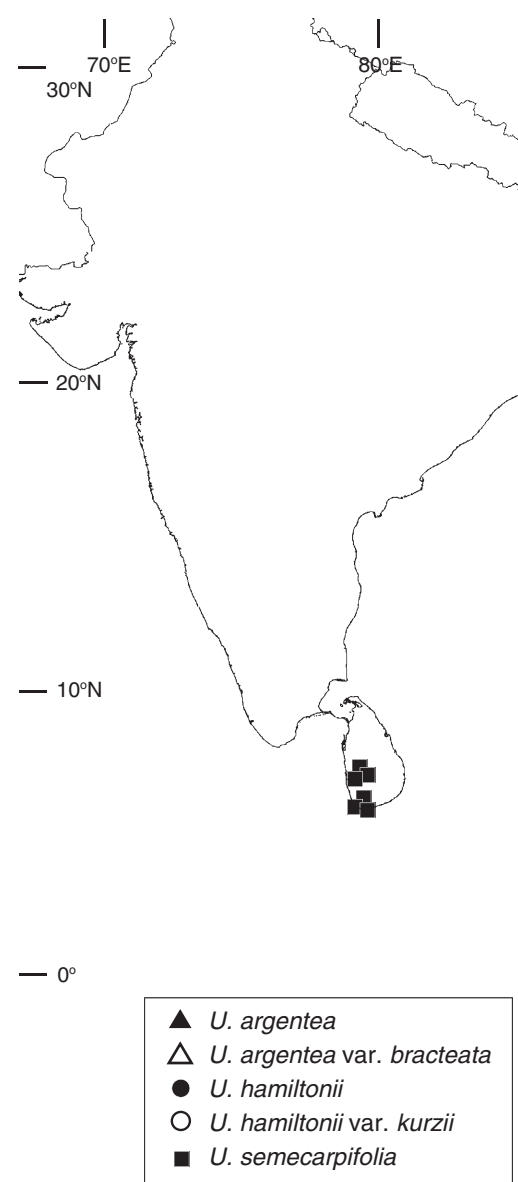

$-10^{\circ} \mathrm{S}$

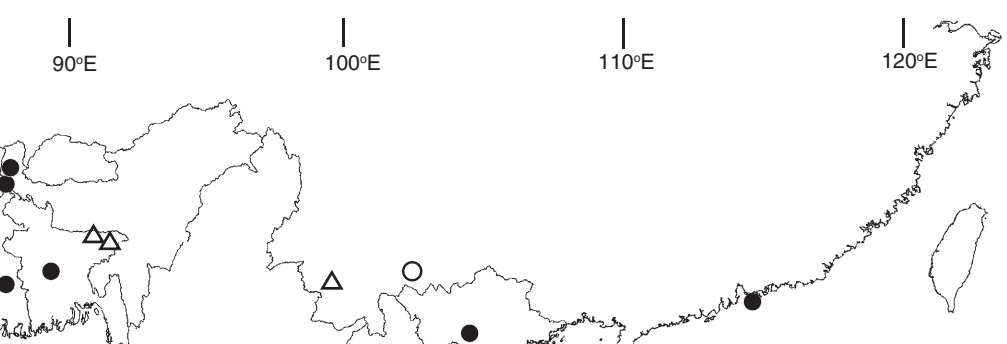

Fig. 11. Distribution of Uvaria argentea, U. argentea var. bracteata, U. hamiltonii, U. hamiltonii var. kurzii and U. semecarpifolia in southern and South-east Asia on the basis of herbarium specimens verified in this study. 


\section{Distribution}

Andaman Islands, central Thailand and Yunnan province, China. Distribution is shown in Fig. 11.

\section{Uvaria macclurei Diels, Notizbl. Bot. Gart. Berlin- Dahlem. (Append.) 11: 73 (1931)}

Type: CHINA. Hainan. Tik Tsok Mau, 18 May 1922, McClure 9697 (lecto, here designated: E 00050326!; isolecto: A 00039699!, BM 000528083!, C 10005706!, G!, N!, P 00142625 !)

Uvaria kweichowensis P.T.Li, Acta Phytotax. Sin. 14: 107 (1976).

Type: CHINA. Kweichow, 8 Aug. 1959, leg. ign. 739 (lecto, here designated: PE 00028383!; isolecto: PE 0028384!, PE 01843406!).

Climber or scandent shrub. Young shoots subtomentose, ferruginous. Leaves elliptical-narrowly obovate, apex acute, shortly acuminate or rarely obtuse, base cuneate-retuse, 110-160 × 40-60 mm, coriaceous; veins (9-)10-12; indument upper surface completely glabrous, shiny, lower blade subglabrous, on midrib and lateral veins hairs 4-6branched, fine, becoming glabrous at maturity. Petiole 3.5-5.5 $\times 1.5-1.75 \mathrm{~mm}$, subtomentose becoming sparse over warty black epidermis. Inflorescence flowers 1 or 2 , peduncle subsessile, 1-2 mm, pedicel $\sim 30-40 \mathrm{~mm}$, basal and medial bracts ovate, $\sim 5.5 \times 4 \mathrm{~mm}$ with prominent veins, often persistent, peduncle, pedicel and bracts subtomentose as for young shoots. Calyx calyptrate in bud, sepals $3, \sim 5-5.25 \times$ 4-4.5 mm, thin and leafy, hairs sparse on inner surface, sparse to subtomentose on outer surface, at length reflexed. Corolla 2 subequal whorls of 3 petals, petals purple, elliptical-acute, 10-12 × 4-5.5 mm, tomentose on outer surface, at length spreading and reflexing, hairs 3-5-branched, sparse on inner surface. Stamens $<50, \sim 3.5 \mathrm{~mm}$, connective apex flattened, somewhat elongate, papillate, prominent dorsal ridge running to base, locules extending to full length of stamen. Carpels $\sim 12-15,3 \mathrm{~mm}$ long, ovary tomentose with flattened spreading hairs, stigma flat and glabrous, U-shaped, ovules 8-10. Fruit monocarps ovoid-ellipsoidal, 40-45 × 30-35 mm, tuberculate, pericarp thick; seeds $6-10$, subsessile, stipe $\sim 6 \times 5 \mathrm{~mm}$, striate.

\section{Distibution}

Rare. Distribution is shown in Fig. 10.

\section{Notes}

The stamens, carpels and petals of $U$. macclurei are similar to those of $U$. rufa; however, the calyx, pedicel and fruit are more similar to those in the $U$. grandiflora group. The species most likely to be confused with $U$. macclurei is $U$. dac, particularly in relation to the fruits; however, in $U$. dac the flowers are much larger, the stamens more numerous and the pedicels much shorter. The lectotypes selected here for $U$. macclurei and $U$. kweichowensis present the best-preserved material from the originally cited sheets.

\section{Uvaria calamistrata Hance, J. Bot. 20: 77 (1882)}

Type: CHINA. Hong Kong, Aug. 1861, Hance 7436 (lecto, here designated: K 000691193!; isolecto: BM 000528082!);
CHINA. Hong-Kong, 10 Aug. 1881, Ford s.n. (syn: K 000691192!)

Climber up to $20 \mathrm{~m}$ tall, or scandent shrub. Young shoots tomentose, becoming mostly glabrous, bark striate with pale lenticels. Leaves broadly elliptic, $70-120 \mathrm{~mm} \times 35-45 \mathrm{~mm}$, apex acute-acuminate-cuspidate, base retuse-cuneate; veins 8-11; indument upper surface of young leaves with sparse pale hairs, becoming glabrous and shiny with maturity, lower surface tomentose to subtomentose, later sparse, hairs stellate, pale, with occasional tufted rufous hairs, especially on veins and midrib. Petiole 5-8 $\mathrm{mm}$ long, tomentose. Inflorescence flowers solitary, 2 or rarely 3 , subopposed, peduncle $2-3 \mathrm{~mm}$, pedicel $\sim 5-10 \mathrm{~mm}$ long, densely tomentose, basally and medially bracteate, one or both bracts often wanting, bracts ovate-obovate-oblanceolate, $\sim 2 \times 1 \mathrm{~mm}$. Calyx calyptrate in bud with apicule, sepals 3 , basally connate, triangular-ovate, apex acute to bluntly acute, up to $5-6 \times 4 \mathrm{~mm}$, membranous, concave, remaining incurved, tomentose on outer surface, sparse on inner surface, often persistent into fruiting stage. Corolla 2 subequal whorls of 3 petals, petals yellow in colour, ovate-oblong, 6-10 × 3-3.5 mm, apex rounded, whorls subequal, partially recurved at maturity, inner and outer surfaces of both whorls tomentose, hairs finer, shorter and more evenly distributed than on inner surface of sepals. Stamens numerous, 1.5-1.8 mm long, connective apex truncate, often obscuring locules completely, papillate, with prominent ridge on external surface, locules extending to full length of stamen, staminodes absent. Carpels $\sim 20-40,1.5 \mathrm{~mm}$ long, ovary tomentose apically and on sides exposed at margin of gynoecium, otherwise with scattered stellate hairs $\sim 0.5 \mathrm{~mm}$ in diameter, stigma U-shaped with elongate lateral slit, $0.2-0.4 \mathrm{~mm}$ long $\times 0.4-0.65 \mathrm{~mm}$ wide, ovules 10-12. Fruit monocarps 2-12, spheroidal-oblongoidal, $\sim 30 \times 20 \mathrm{~mm}$, initially covered with prominent beard of 5-8 $\mathrm{mm}$ long membranous bluntly triangular outgrowths, later tuberculate with scattered remnants of outgrowths over surface, stipe short, $3-5 \mathrm{~mm} \times$ 2.5-3mm; seeds 1-12, 12 $12 \times 4 \mathrm{~mm}$, ellipsoid, to hemiellipsoidal, smooth, ruminations faint, hilum $5 \times 4 \mathrm{~mm}$, ovate.

\section{Distribution}

Uvaria calamistrata occupies a range of habitats similar that of to Uvaria boniana, particularly in Hainan, where many specimens have been collected scrambling over rocky scrub on steep hillsides. Distribution is shown in Fig. 12.

\section{Notes}

The bearded juvenile fruit of $U$. calamistrata is among the most distinctive in the genus. The beard is very different from that seen in Uvaria vietnamensis Meade, being not hair-like and not persistent. The lectotype selected presents the bestpreserved material from the originally cited sheets.

\section{Uvaria zeylanica L., Sp. Pl. 536 (1753)}

Type: SRI LANKA. s. loc., leg. ign. (lecto: BM (Herb. Hermann) 5: 163, t. 224!. First step lecto fide H.Huber in M. D.Dassanayake \& F.R.Fosberg (eds), Rev. Handb. Fl. Ceylon 5: 17 (1985); second step lecto fide P.J.A.Kessler in K.Kubitzki et al. (eds), Fam. Genera Vasc. Pl. 2: 97 (1993)). 'Uvularia. Uva 


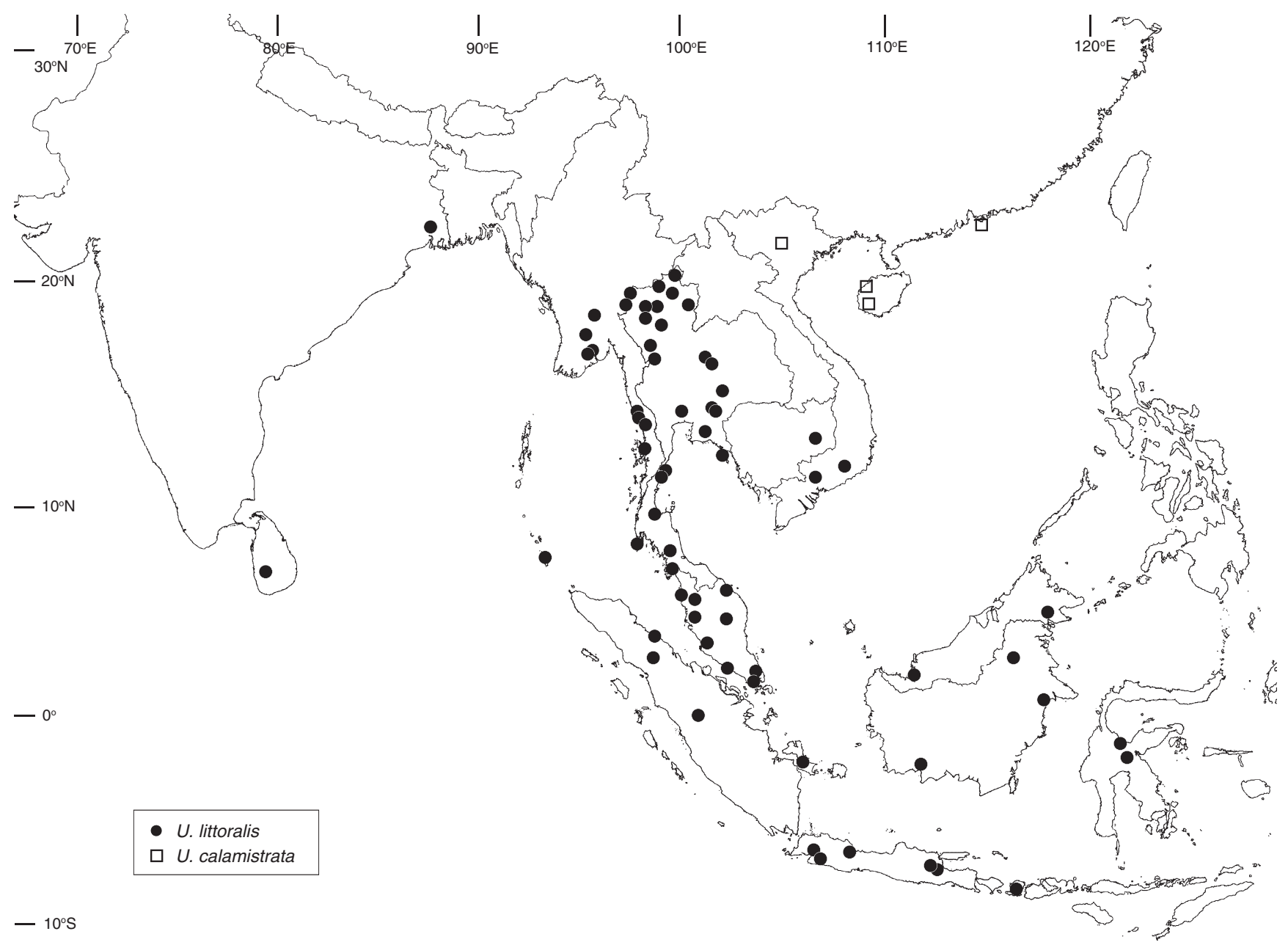

Fig. 12. Distribution of Uvaria calamistrata and $U$. littoralis in southern and South-east Asia on the basis of herbarium specimens verified in this study.

zeylanica. 224. (syn: BM 000621773 (Herb. Hermann)!); 'Narum panel. Hort. Malab. tom. 2 p. 11. Balla paleti. Calo Apocaro. Uves de mato. 224' (syn: BM 000621836 (Herb. Hermann)!).

Uvaria coriacea Vahl, Sym. Bot. 3: 72 (1794).

Type: SRI LANKA. s. loc., s. dat., J.G.K.Konig s.n. (lecto: C 10005711, fide I.M.Turner, Nordic J. Bot. 33: 293 (2015)).

Guatteria malabarica Dunal, Monogr. Anonac. 134 (1817).

Type: 'Kaltsjerou-Panel' in Rheede Tot Draakenstein, Hort. Malab. 5: t. 17 (1685) (lecto, fide I.M.Turner, Nordic J. Bot. 33: 293 (2015)); Guatteria montana DC., Syst. Nat. 1: 508 (1817), nom. illeg.

Uvaria lutea Wall., Numer. List no. 6462 (1832), nom. inval., nom. nud.

Habit climber up to $30 \mathrm{~m}$ tall or sarmentose shrub 1-2 m high. Young shoots subtomentose, smooth, quickly becoming striate and subglabrous. Leaves ovate-elliptic-oblanceolate, base cuneate, apex acute-acuminate, $~ 55-70(-95) \times$ 20-28(-35) mm, membranous, waxy; veins $\sim 8-12$; indument blades glabrous with occasional stellate hairs on midrib and vein bases. Petiole $\sim 2-3 \times 1 \mathrm{~mm}$, tomentose when young, sparse when mature, hairs ferruginous, surface black, waxy. Inflorescence terminal or leaf-opposed by apposition, flowers solitary or less often 2-3, peduncle $1.5-2 \mathrm{~mm}$ pedicel (4-)5(-7) mm, densely tomentose, hairs pale or ferruginous, bracts narrowly ovate-elliptical, membranous. Calyx calyptrate in bud, tearing into 3 subequal ovate-acute to broadly lanceolate membranous sepals, outer surface tomentose, later sparse, inner surface subglabrous, sepals persistent. Corolla 2 subequal whorls of 3 petals, petals dark red-purple in colour, narrowly elliptical-oblong, apex acute or less often narrowly obtuse, $\sim 10 \times 3 \mathrm{~mm}$, membranous, somewhat warty, inner surface subglabrous, outer surface sparse to subtomentose with 1-5-branched straggling pale hairs, margins and apex tomentose, both surfaces. Stamens numerous, $\sim 1 \mathrm{~mm}$ long, cuneate, locules extending full length of stamen, connective glabrous, broadly concave, undulating. Carpels $\sim 20,1 \mathrm{~mm}$ long, cuneate, ovary hairy, stigma glabrous, U-shaped, lobes broad, $\sim 0.6 \mathrm{~mm}$ in diameter, ovules $\sim 6$. Fruit monocarps $6-12$, shortly oblong, apex and base rounded, $\sim 12 \times 8.5 \mathrm{~mm}$, densely and shortly tomentose, hairs ferruginous, blunt, pericarp thin in young fruits, later thickening, becoming fleshy, edible, stipe short, $\sim 3 \times 1.25 \mathrm{~mm}$; seeds $\sim 6$ in 2 rows, hemispheroidal or 
flattened hemi-ellipsoidal, $\sim 4 \times 3 \times 2 \mathrm{~mm}$, testa brown, smooth and shiny, ruminations visible. (Fig. 1D, 5.)

\section{Distribution}

In Sri Lanka, this species usually grows as a scrambling shrub in dry regions and as a medium to large climber in the wetter forested regions, usually in secondary vegetation. Huber (1985) noted that the species is most common near the coast, often on sand-dunes. In southern India $U$. zeylanica is confined to moist lower hill forests. Distribution is shown in Fig. 10.

\section{Notes}

Uvaria zeylanica is the type species for the genus. Linnaeus confused it with the 'narum-panel' of Rheede (later Unona narum Dunal) from the Malabar coast, although the specimens and plate that he described from Hermann's Musaeum zeylanicum (Hermann 1717) are of U. zeylanica only. Kessler (1993) lectotypified U. zeylanica on the basis of a plate from this collection; however, only the Hermann isolectotypes at BM actually illustrate floral structure.

\section{Uvaria sphenocarpa Hook.f. \& Thomson, Fl. Ind. 1: 99 (1855)}

Type: SRI LANKA. s. loc., s. dat., Thwaites 1045 (lecto: K 000691186!; isolecto: BM 000528053!, G 00226154!, P 00142689-90!, P 00248403!, P 01989167!, TCD 0018020!, US 01049781!, fide I.M.Turner, Nordic J. Bot. 33: 293 (2015)).

Habit climber or sarmentose shrub. Young shoots ferruginotomentose, hairs becoming sparse, bark black, smooth at first, later grey-black and striate, hairs persistent in grooves. Leaves elliptic to narrowly obovate, base retuse to shortly cordate, apex acuminate, $70-140(-200) \times 25-40(-70) \mathrm{mm}$, acuminum up to $30 \mathrm{~mm}$, subcoriaceous; veins $~ 9-10$; indument uppersurface hairs 1-8-branched, sparse, becoming subglabrous, lower blade subtomentose to sparse, hairs larger, more rufous than above, 4-10-branched. Petiole $\sim 4-5 \times 1.5-2 \mathrm{~mm}$, subtomentose to sparse with large ferruginous hairs. Inflorescence leaf-opposed, flowers solitary or in short 2- or 3 -flowered cymes, usually only 1 bud in flower at any one time, peduncle subsessile, pedicel $\sim 3-4 \mathrm{~mm}$, bracts clasping, ovate, $\sim 4 \times 2 \mathrm{~mm}$, pedicel and bracts with dense ferruginotomentose indument. Calyx sepals 3 , basally connate, opening early, $\sim 4.5 \times 2.5 \mathrm{~mm}$, broadly ovate, indument ferruginotomentose on outer surface, sparse at margin, on inner surface glabrous at base, sparse elsewhere. Corolla 2 subequal whorls of 3 petals, petals pale yellow in colour, elliptical-obovate, obtuse or bluntly acute, $\sim 6-6.5 \times$ 4-4.5 mm, spreading, tomentose on outer surface, subtomentose on inner surface. Stamens $\sim 50+, 2 \mathrm{~mm}$ long, all fertile, connective head broad and flat, somewhat $\mathrm{T}$-shaped, very shortly papillate, dorsal ridge prominent, locules full length. Carpels $\sim 50,2 \mathrm{~mm}$ long, $0.5 \mathrm{~mm}$ wide, ovary tomentose, stigma U-shaped, lobes large, copious nectar often present, ovules $\sim 4$. Fruit monocarps sessile, $\sim 10-16$, yellowish, gathered into pseudo syn-carpous conglomerate, individual monocarps cuneate-obovoidal, 18-25 $\times$ 15-20 mm, sides flattened against adjacent monocarps, all surfaces densely tomentose, apical surface coarsly warted; seeds $\sim 4,10 \times 5 \times 3 \mathrm{~mm}$, smooth, brown.

\section{Distribution}

Endemic to Sri Lanka, occurring in wet and dry zones from sea level to $\sim 700 \mathrm{~m}$. Huber (1985) noted that it has become more common owing to disturbance of primary forest. Cultivated at Bogor. Distribution is shown in Fig. 9.

\section{Notes}

The flowers and leaves of $U$. calamistrata can be similar to those of $U$. sphenocarpa Hook.f. \& Thomson.

\section{Uvaria semecarpifolia Hook.f. \& Thomson, FI. Ind. 1:97} (1855)

Type: SRI LANKA. s. loc., s. dat., G.W. Walker s.n. (lecto: K001096932!, fide I.M.Turner, Nordic J. Bot. 293 (2015)); SRI LANKA. s. loc., s. dat., Thwaites 244 (syn: BM 000528050!, G 00226155!, K!, L 1768675!, P 00751048!, P 00142683!, P 00753701!, P 00248400-1!, TCD 0018019!): syn: W. Griffith s.n. (K!).

Large climber up to $30 \mathrm{~m}$ tall. Young shoots densely tomentose, shoots becoming woody and ridged quickly, indument retained in grooves, glabrous on ridges, lenticels large and numerous. Leaves elliptic to oblong, base cordate, apex obtuse to cuspidate, $160-230(-450) \times 90-110(-120) \mathrm{mm}$, coriaceous; veins $\sim 16-20(-25)$; indument upper blade completely glabrous and shiny, hairs on midrib erect, tomentose, 1-4-branched, up to $1 \mathrm{~mm}$ long, lower blade hairs sparse to subtomentose, 3-8-branched. Petiole thick, 7-10 $\times$ 3-4 mm, ferrugino-tomentose, epidermis warted below into transverse foliations. Inflorescence leaf-opposed, flowers 2 or 3, peduncle $\sim 6-7 \times 3 \mathrm{~mm}$, flattened in tranverse section, pedicels $\sim 10 \mathrm{~mm}$, with basal $3 \mathrm{~mm}$ adnate to sympodial axis, densely tomentose, bracts broadly obovate, up to $\sim 4 \times 6.5 \mathrm{~mm}$, densely tomentose outside, glabrous within, often wanting. Calyx connate, splitting apically in early bud at $\sim 5-6 \mathrm{~mm}$ in diameter, later forming connate ring, finally splitting into 3 sepals before opening of corolla, sepals ovate-acute, $\sim 8 \times 4 \mathrm{~mm}$, fleshy, shortly ferrugino-tomentose outside, glabrous within. Corolla 2 whorls of 3 petals, outer petals spreading, inner petals connivent, petals green in colour, maturing yellow-brown, outer whorl spreading, $\sim 9 \times 7 \mathrm{~mm}$, broadly ovate, outer surface shortly and densely tomentose, hairs finer than on calyx, inner petals connivent, obovate, basally constricted or shortly clawed, $\sim 8 \times$ $6 \mathrm{~mm}$, indument as for outer whorl. Stamens numerous, $1.5-2 \mathrm{~mm}$, tubular, connective concave, prominently papillate, locules full length. Carpels $\sim 20-25,2.5 \mathrm{~mm}$ long, ovary tomentose, somewhat flattened apically, stigma U-shaped, glabrous, ovules $\sim 8$. Fruit monocarps $\sim 10-20$, broadly ellipsoidal to obovoid, $\sim 30-40 \times 20-30 \mathrm{~mm}$, shortly and densely tomentose, surface tubercled or undulating, pericarp $\sim 4 \mathrm{~mm}$ thick in unripe specimens, stipe $\sim 3 \times 10-15 \mathrm{~mm}$; seeds $\sim 8$, up to $12 \times 10 \times 6 \mathrm{~mm}$.

\section{Distribution}

Endemic to lowland rainforest in the wetter parts of southwestern Sri Lanka. Distribution is shown in Fig. 11. 


\section{Notes}

Uvaria semecarpifolia can be distinguished from the similar $U$. littoralis by its tomentose fruits, more oblong leaves with 20 parallel veins and its juvenile flowers with a connivent inner whorl.

13. Uvaria narum (Dunal) Blume, Fl. Javae 21, 22: 5 (1830)

Unona narum Dunal, Monogr. Anonac. 99 (1817).

Type: 'Narum-panel' in Rheede Tot Draakenstein, Hort. Malab. 2, t. 10 (1679). SRI LANKA. Uvaria zeylanica enc. no. 3, Narum-panel Rheed. Mal. 2 t. 10, s. dat., leg. ign., s.n. (epi, here designated: P-JU!).

Uvaria narum Wall., Numer. List no. 6473 (1832), nom. inval., nom nud. Uvariaflexuosa Wall., Numer. Listno. 6473b (1832), nom. inval., nom. nud. Uvaria malabarica Oken, Allg. Naturgesch. 3(2): 1254 (1841).

[Uvaria zeylanica auct. non L.: J.B.A.P.M. de Lamarck, Encycl. 1: 596 (1785); A.P.Candolle, Syst. Nat. 1: 481 (1817), p.p.].

Habit sarmentose shrub or climber. Young shoots black, very finely striate, (sub)glabrous, hairs $\sim 0.2 \mathrm{~mm}$ in diameter.
Leaves oblong-elliptical-oblanceolate, base cuneate, apex acute-acuminate, $\sim 145 \times 45 \mathrm{~mm}$, acuminum up to $15 \mathrm{~mm}$, subcoriaceous, waxy; veins $\sim 10-11$; indument rare spreading hairs on very young leaves, quickly becoming glabrous above and below. Petiole $\sim 4-5 \times 1 \mathrm{~mm}$, glabrous, transverse ridges prominent below, minutely tubercled. Inflorescence terminal or leaf opposed, flowers solitary, buds globose, peduncle $\sim 4 \mathrm{~mm}$ long, pedicel $\sim 20-30 \mathrm{~mm}, 0.75-0.85 \mathrm{~mm}$ in diameter, broadening at receptacle, indument sparse to subtomentose, hairs rufous, many-branched, bracts membranous, ovate-elliptical, $\sim 3 \times 1.5 \mathrm{~mm}$, subglabrous to sparse. Calyx valvate, sepals 3 , broadly ovate, $\sim 6-8 \times 3.5-4.5 \mathrm{~mm}$, concave, reflexing at maturity, outer surface subglabrous and minutely tuberculate with ferrugino-tomentose margin and apex, inner surface densely and shortly tomentose with pale shaggy stellate hairs. Corolla petals 6 , basally connate, usually in 2 whorls of 3, or rarely fused in a single whorl, petals blood-red in colour, membranous, outer whorl 10-12 × 9-11 mm, inner whorl 8-11 × 7-9 mm, all petals initially broadly ovate-acute, maturing obovate, obtuse-acute, concave, reflexed at maturity, shed as a ring, both surfaces of both whorls shortly tomentose with fine, straggling pale hairs, $\sim 0.2 \mathrm{~mm}$ in diameter. Stamens

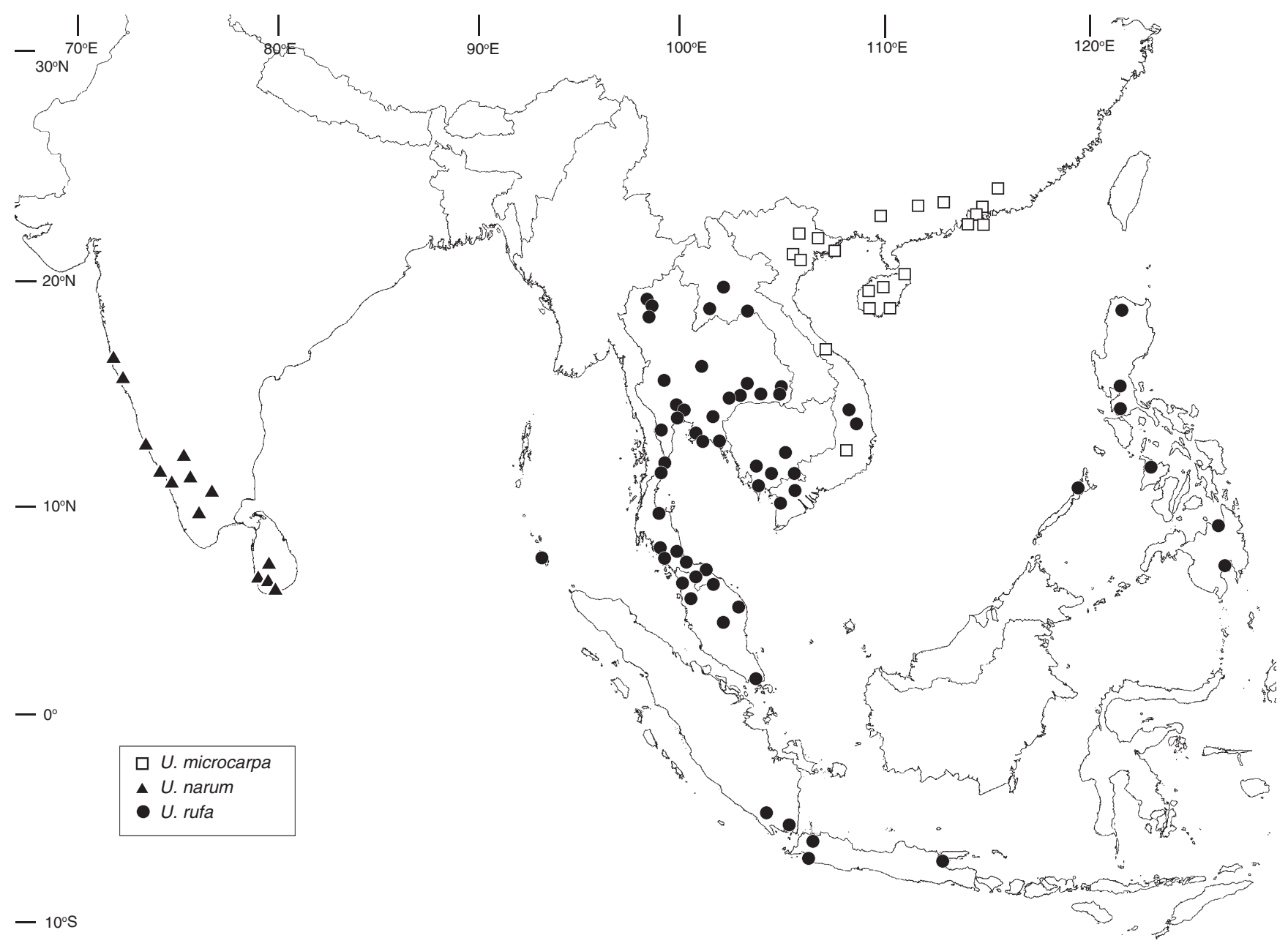

Fig. 13. Distribution of Uvaria microcarpa, U. narum and U. rufa in southern and South-east Asia on the basis of herbarium specimens verified in this study. 
numerous, $\sim 1.75 \mathrm{~mm}$ long, connective apex globose, very shortly papillate, locules extending to full length of stamen, staminodes absent. Carpels numerous, $\sim 2 \mathrm{~mm}$ long, ovary hairy, stigma U-shaped, glabrous, black, often covered with exudate, ovules 6 . Fruits 4-20+, green turning orange then red, monocarps globose to oblong, 10-15 × 6-8 mm, surface subglabrous to glabrous, granular or minutely tuberculate, pericarp fleshy at maturity, drying thin, stipe narrow, $\sim 25-35$ $\times 1 \mathrm{~mm}$ in diameter, subglabrous with scattered pale stellate hairs $\sim 0.2 \mathrm{~mm}$ in diameter; seeds $2-6, \sim 5.5 \times 4 \times 3 \mathrm{~mm}$, compressed ovoid, ruminations visible, raphe darker than remainder of testa, aril not prominent, 2 rows packed closely together. (Fig. 3.)

\section{Distribution}

A common species near watercourses on the wet coastal plains and hills of south-western Sri Lanka and the Malabar coast. Distribution is shown in Fig. 13.

\section{Notes}

Uvaria narum was the first taxon in the genus to receive attention from European botanists, most notably from van Rheede Tot Draakenstein (1679) who described and illustrated it as 'Narumpanel' in his Hortus Malabaricus. Linnaeus listed Rheede's Narum-panel as a synonym of $U$. zeylanica. Later Dunal differentiated between Rheede's Narum-panel and Linnaeus' U. zeylanica, renaming the former Unona narum. He indicated that de Lamarck (1785) had misapplied the name U. zeylanica L. to Unona narum Dunal. The material on which de Lamarck based his description of $U$. zeylanica is held in the Jussieu family herbarium in Paris, and has been selected here as an epitype for $U$. narum.

This species is often confused with $U$. concava, and, to a lesser extent, $U$. macropoda. Both have a strongly tuberculate calyptrate calyx with a prominent apicule, whereas the calyx of $U$. narum is valvate and covered with a short subtomentose indument of fine rufous hairs. The mature monocarps of $U$. narum are much smaller than those of $U$. concava, have a pericarp that becomes very thin on drying, and are mounted on a short, narrow stipe. The fruits of $U$. concava are variously thickwalled, usually large, carrying one or more longitudinal ridges on the monocarp surface, and are mounted on a long, thickened stipe. The monocarps of U. macropoda are narrowly cylindrical, tomentose, have a thick apicule and are mounted on stipes up to twice as long and broad as those of $U$. concava.

\section{Uvaria littoralis (Blume) Blume, Fl. Javae 21, 22: 26} (1830)

Unona littoralis Blume, Bijdr. fl. Ned. Ind. 1: 16 (1825).

Type citation: 'in Bataviae locis stagnosis.'

Type: INDONESIA. Java, s. dat., s.n. 'Unona littoralis Bl.' Blume leg. (lecto, here designated: U-1074978!) (syn: BO n.v.).

Uvaria macrophylla Roxb., Hort. Bengal. 43 (1814), nom. inval., nom. nud.

Guatteria cordata Dunal, Monogr. Anonac. 129 \& pl. 30 (1817); Uvaria cordata (Dunal) Wall., Numer. List no. 6486 (1832), nom. illeg., non Schumach. \& Thonn. (1827).
Type: 'Guatteria cordata' in M.F.Dunal, Monogr. Anonac. pl. 30 (1817). MALAYSIA. Penang, s. dat., G.Porter in Wall. Cat. no. $6486 A$ (epi, here designated: K-W 001124009!).

Uvaria macrophylla Roxb. ex Wall., Pl. Asiat. Rar. 2: 22, t. 122 (1830).

Type: INDIA. Cultivated in Calcutta Botanic Garden, s. dat., Wall. Cat. 6487A (lecto: K 000442833!; isolecto: BM 000528071!, fide I.M.Turner, Phytotaxa 36: 102 (2011)).

Uvaria macrophylla Roxb., Fl. Ind. Ed. 1832 2: 663 (1832), isonym.

Habit large climber, reaching $30 \mathrm{~m}$. Young shoots densely ferrugino-tomentose, becoming striate and glabrous. Leaves broadly ovate-oblong(-obovate), base cordate to subcordate, apex obtuse or shortly acuminate, rarely obcordate, $\sim 200-300 \times$ 150-180(-200) mm, coriaceous; veins 5-25+, often basally branched or merging; indument upper blade subglabrous, midrib and main vein traces densely tomentose, lower blade and veins tomentose to subtomentose. Petiole $\sim 10 \times 3.5 \mathrm{~mm}$, ferrugino-tomentose, adaxial groove deep. Inflorescence 2-5+ buds in tightly packed rhipidium, up to 3 open at any time, compound peduncle 5-10(-15) $\mathrm{mm}$ long, 2-3 $\mathrm{mm}$ in diameter, flattened in transverse section, densely ferrugino-tomentose, pedicel $3-10 \mathrm{~mm} \times 2-2.5 \mathrm{~mm}$, flattened with shallow groove on inner surface, basally and medially bracteate, bracts broadly obovate, rarely ovate, fleshy, up to $5 \times 7 \mathrm{~mm}$ in diameter, remaining concave and wrapping around pedicel, often enclosing younger buds, ferrugino-tomentose. Calyx connate in early bud then splitting apically to form a basally connate three-pointed ring around bud, ring later splitting into 3 ovate-acute sepals, 4-6 6 6-8 mm wide. Corolla petals 6, basally connate in a single whorl, petals red, fleshy, ovateacute, usually distinctly concave, expanding to $15-18 \times$ 10-12 mm, reflexing at maturity, outer surface shortly tomentose, inner surface hairs shorter, villose. Stamens numerous, 5-6 mm long, connective apex elongate, 5-sided in central whorls, flat in outer whorls, minutely papillate, locules full length, decreasing in size to staminodal outermost whorl. Carpels 20-25, 4-4.25 mm long, indument stellate, short and somewhat combed, stigma short, glabrous, U-shaped, base narrowed and subtomentose, ovules 12-14. Fruit monocarps 10-20, spherical-ovoid-oblong, younger fruits bright green, subglabrous, often with constrictions between seeds, mature fruits $\sim 30 \times 16.5 \mathrm{~mm}$, usually glabrous, orange or yellow turning maroon red, ripening black with soft pericarp, stipe 2-5 × 2-3 mm; seeds 10-14, hemi-ellipsoidal, $10 \times 7 \times 3 \mathrm{~mm}$.

\section{Distribution}

This is a morphologically variable species, particularly in the shape and indument of the leaves, found mainly in mixed and evergreen forests below $300 \mathrm{~m}$, but also occurring in fire-prone or disturbed deciduous dipterocarp forest. Distribution is shown in Fig. 12.

\section{Notes}

The earliest legitimate epithet for this taxon at species rank is cordata, but this is pre-occupied in Uvaria by Uvaria cordata Schumach. \& Thonn. (1827) (=Uvaria ovata DC.). Blume's later name, Unona littoralis, therefore has priority. The type sheet at Bogor has not been seen; however, the Blume specimen 
from Java at Utrecht (U 1074978, ex BO), also annotated by Blume, is a clear match for his type description, and is here designated as a lectotype.

The type material that Dunal mentioned in the original description for Guatteria cordata ('Uvaria zeylanica Herb. Deless.') appears to have been lost or destroyed. There are specimens of Uvaria zeylanica in Herb. Deless. in Geneva; however, because these are annotated by Dunal as types for $U$. dulcis Dunal and U. javana Dunal, they do not constitute original material of $G$. cordata. As a result, the plate accompanying the description is the sole original material remaining. The earliest surviving sheet identified on collection as $G$. cordata following Dunal's publication in 1817 is in Cat. 6486A at the Wallich herbarium, collected by G. Porter in Penang. Because it comprises material with both flowers and fruit, it is here designated as an epitype.

15. Uvaria microcarpa Champ. ex Benth. Hooker's J. Bot. Kew Gard. Misc. 3: 256 (1851)

Uvaria macrophylla var. microcarpa (Champ. ex Benth.) Finet \& Gagnep., Bull. Soc. Bot. France (Mém. 4 (2)) 53: 67 (1906).

Type: CHINA. Hong Kong, 1850, Champion 39 (lecto, here designated: K 000691191 !); CHINA. Hong Kong, s. dat., Champion s.n. (syn: K 000691190 !).

Uvaria badiiflora Hance, Ann. Bot. Systemat. 2: 19 (1851).

Type citation: 'Hab. in ins. Hongkong Sinarum.'

Type: n.v., lost or destroyed?

Scandent shrub or climber. Young shoots ferruginotomentose, becoming striate and glabrous, sometimes flattened and broad. Leaves elliptic-broadly obovate, base cordate or broadly retuse, frequently oblique, apex acuterounded, $\sim 80-150 \times 40-80 \mathrm{~mm}$, thickly coriaceous, blade often undulate at margin, veins 9-11(-12); indument hairs stellate, tomentose and erect on upper surface of midrib, sparse to glabrous on veins and blade, hairs on lower surface fine, straggling, subtomentose-sparse. Petiole short and thickened, 5-6 $62 \mathrm{~mm}$, shortly and sparsely ferruginotomentose, black and warty underneath. Inflorescence leafopposed, flowers 1-2(-3), peduncle 1.5-3.5(-10) mm, pedicel $\sim 10-15 \times 1.4-1.8 \mathrm{~mm}$, both sometimes flattened in transverse section, bracts elliptic-oblong, 3-5 × $-3 \mathrm{~mm}$, veins prominent, peduncle, pedicel and bracts all shortly ferrugino-tomentose. Calyx connate in early bud, then splitting apically to form a basally connate three-pointed ring around bud, ring later splitting into 3 triangular or broadly ovate $\sim 5-7 \times 5-10 \mathrm{~mm}$ sepals, remaining concave, sometimes reflexed, often persistent as connate ring, indument ferrugino-tomentose on outer surface, less dense on inner surface. Corolla 2 whorls of 3 petals, inner petals slightly smaller than outer, petals brown or red, fleshy, ovate-broadly elliptical, obtuse-bluntly acute, apex often incurved, inner whorl $\sim 10-12 \times 7-8 \mathrm{~mm}$, outer whorl $\sim 10-12 \times 8-9 \mathrm{~mm}$, inner and outer petals erect at first, later recurved, surface warty becoming smoother at maturity, densely and shortly tomentose on both surfaces. Stamens numerous, 4.5-6 mm long, glabrous, connective apex papillate, flattened, becoming progressively longer and broader towards external whorl, on inner whorl locules extending full length of stamens, outer whorl semi-staminodal with small basal locules $\sim 0.5-1 \mathrm{~mm}$ long. Carpels numerous, $\sim 4 \mathrm{~mm}$ long, in dense whorl, ovary ferrugino-tomentose, stigma $\sim 1 \times 0.5 \mathrm{~mm}$, glabrous, U-shaped, lobes somewhat broadened, ovules $\sim 6-8$ in 2 rows. Fruit monocarps $4-8+$, green turning yellow then dark purple at maturity, ovoid to shortly oblongoid, $\sim 20 \times$ $10 \mathrm{~mm}$, pericarp thin, stipe $\sim 10-15 \times 1-2 \mathrm{~mm}$, shortly tomentose; seeds $\sim 6-8$, flattened-ovoid, $\sim 8 \times 8 \times 2 \mathrm{~mm}$, dark brown, smooth, shiny, ruminations visible, aril $\sim 4 \times$ $2 \mathrm{~mm}$, hilum circular-ellipsoid $\sim 1 \mathrm{~mm}$ in diameter. (Fig. 1D.)

\section{Distribution}

Uvaria microcarpa shows a typical Indochina-southern China distribution and is often found as a scandent or erect shrub in open vegetation. However, it is most common growing as a climber in areas of (semi-)intact forest, usually near watercourses or on hillsides. In the Lianhua Shan Range $(22.5 \mathrm{~N})$, it is found at altitudes of up to $1500 \mathrm{~m}$. Distribution is shown in Fig. 13.

\section{Notes}

This species and $U$. littoralis are often confused; however, their inflorescence structure differs, with $U$. microcarpa having one or two flowers mounted on a narrow elongate pedicel with small elliptical bracts, and the peduncle, where developed, is narrow and circular in transverse section. Furthermore, in $U$. littoralis the flowers are mounted on a closely packed cyme of $2-5+$ pedicels and the peduncle is short and broad, usually having a prominent abaxial groove. The petals of $U$. littoralis are typically concave-acute and bright red in colour; those of $U$. microcarpa are flat or spreading, broadly elliptical and red to brown in colour. The fruits of $U$. microcarpa are considerably smaller and carry fewer seeds than those of $U$. littoralis. Uvaria microcarpa also tends to have smaller leaves, and the presence of any large leaves is usually a good indication that the specimen is $U$. littoralis. The two species occupy different geographic ranges, namely, $U$. littoralis west and south of the Mekong and $U$. microcarpa north and east of it. Uvaria littoralis is present in the Mekong Delta and probably also in Cambodia. The most southerly record for U. microcarpa is in Dac Lac Province, inland from Da Lat. McClure (collection number 638, Guandong Province, China, 5 January 1932, NY!; see the Full list of material examined section in the Supplementary material) reported that the leaves are used to make yeast cakes ('Tsau peng'). The lectotype selected presents the best-preserved material from the originally cited sheets.

\section{Uvaria rufa Blume, Fl. Javae 21, 22: 19 t. 4, 13C (1830)}

Type: INDONESIA. Java, s. dat., Blume s.n. (lecto, heredesignated: P 00248470!) (syn: BO n.v., K 000380303!, L!, P 00248468-69!).

Guatteria rufa Dunal, Monogr. Anonac. 129, t. 29 (1817).

Type: INDONESIA. Annona (Uvaria) tomentosa, herbier de l'Inde de M. Poivre, Java, Jussieu Cat no. 10795 + B, ab Uvaria tomentosa Roxb t.35, differ follis basi subcordati, s. dat., Poivre 
s.n. (lecto, here designated: P-JU 00662855 !); INDIA. Cananga? tomentosa D.C., Uvaria tomentosa occurit in fallor antea sub numonae Annona tomentosa, indie, Lahaye s.n., Herb. Juss. Cat. $10795+A$ (syn: P-JU 00662853!); INDONESIA. Guatteria rufa, isle de Timor, voyage Captain Boudin, s. dat., Riedlé s.n. (syn: P 00142680!, G!).

Uvaria astrosticta Miq., Fl. Ned. Ind., Eerste Bijv. 370 (1860).

Type: INDONESIA. Sumatra, Lampang, Tarabangie, s. dat., Teijsmann s.n. 4261 H.B. (lecto, here designated: U 0000436!); INDONESIA. Sumatra, Bepisang, Tigo Nemrin, s. dat., Teijsmann 4338 H.B. (syn: U 0000435!).

Uvaria ridleyi King, J. Asiat. Soc. Bengal Pt. 2, Nat. Hist. 61(1): 19 (1892).

Type: MALAYSIA. Panang, s. dat., Ridley s.n. (lecto, here designated: $\mathrm{K} !)$.

Uvaria hamiltonii var. fauveliana Pierre ex Finet \& Gagnep., Bull. Soc. Bot. France (Mém. 4 (2)) 53: 68 (1906); Uvaria fauveliana (Pierre ex Finet \& Gagnep.) Pierre ex Jovet-Ast, Not. Syst. 9: 87 (1940), p.p.

Type: VIETNAM. Phu Quoc, hab in montibus, Raong Chiao, Mar. 1874, Pierre 1787 (lecto, here designated: P 00248362!; isolecto: BM 000949957!, K 000380684!, P 00248363!).

Habit shrub or large woody climber up to $25 \mathrm{~m}$ high. Young shoots ferrugino-tomentose, hairs becoming blunt and sparse, bark smooth, becoming striate with prominent lenticels. Leaves elliptic-narrowly obovate, base retuse-subcordate, apex acute, 100-175 × 35-75 mm, blade rigid, subcoriaceous; veins 10-13; indument on upper blade sparse, hairs 2-5-branched, erect, scabrid, longer over midrib and veins forming prominent ferrugino-tomentose ridge, lower blade hairs 9-12-branched, subtomentose, blunt on midrib and veins. Petiole $\sim 4-5 \times$ $1.5 \mathrm{~mm}$, ferrugino-tomentose. Inflorescence leaf-opposed, cymose with up to 4 pedicels, each bearing a single floral bud, with only the oldest 1 or 2 bearing mature flowers at any one time, peduncle $\sim 3 \times 1.5 \mathrm{~mm}$, pedicels $\sim 8-10 \mathrm{~mm}$, bracts $\sim 6 \times 4.5 \mathrm{~mm}$, ovate, apex acute-rounded, palmate veins visible on outer surface, peduncle and pedicel with ferruginotomentose hairs, hairs sparse and shorter on bracts. Calyx sepals 3, valvate at apex, connate basally, broadly triangular, $\sim 6 \times 3 \mathrm{~mm}$, apex acute-obtuse, indument ferruginous on inner and outer surfaces, forming distinct ridge at margin, often persistent, forming detached ring at base of fruiting receptacle. Corolla 2 subequal whorls of 3 petals, petals red or dark purple in colour, broadly elliptic-oblong, apex rounded, $\sim 12-14 \times 5-6 \mathrm{~mm}$, fully reflexed at maturity, indument sparse, fine and shaggy. Stamens $\sim 30-40$, 3-3.5(-4) long $\times 0.5 \mathrm{~mm}$ wide, flattened, connective cap up to $1 \mathrm{~mm}$ long, papillate, on inner whorl locules extending full length of stamen, submarginal whorl of stamens broad and flat and staminode-like with much reduced locules, outer whorl staminodes, $\sim 14$ in number, $3 \mathrm{~mm}$ long $\times 1.5-2 \mathrm{~mm}$ wide, broadly obovate and flattened. Carpels $\sim 20$ or fewer, $3.75 \times 0.75 \mathrm{~mm}$., ovary tomentose, hairs flattened, stigma U-shaped and glabrous but occluded on three sides by extension of ovary wall above a distinctly narrowed neck, ovules $~ 24$ in two lateral rows. Fruit monocarps 4-8(-20), cylindrical, 25-35 $\times 14-21 \mathrm{~mm}$, young fruits green with rufous tint, pericarp thick and hard, mature fruits bright red in colour, flesh white, juicy, pericarp wall subglabrous, thin and easily torn, stipe ridged, elongate, $\sim 25-30 \times 1-2 \mathrm{~mm}$, indument sparse, hairs spreading; seeds 5-14(-20+), $6 \times 4 \times 2 \mathrm{~mm}$, compressedellipsoidal, ruminations visible, in 2 rows, occasionally compressed into what appears to be 1 row. (Fig. 1D, E, 4.)

\section{Distribution}

Probably the most common species of Uvaria in continental Asia. Although it is found in most forested habitats (although only occasionally in thickets in open dipterocarp forest), it is most common in dry evergreen and mixed deciduous types, and occurs in remnant forest vegetation. Distribution is shown in Fig. 13.

\section{Notes}

Uvaria rufa is most easily distinguished by the rough texture of the abaxial leaf surface and the outer whorl of broad and flat staminodes. The sweet tangy fruit is valued throughout much of Indochina and, although it is not actively cultivated, individual wild plants are often well known to local communities. The species is used in traditional medicine, particularly for the treatment of stomach cramp and period pain. Dunal (1817) first described this taxon as Guatteria rufa. Blume made reference to the latter in his description of U. rufa, but did not specifically mention it as a synonym, as he did with U. javana, for example. But Dunal's epithet 'rufa' cannot be transferred to Uvaria because of the presence of Blume's heterotypic taxon; therefore, Blume's name is affirmed here as the legitimate one for the taxon within the genus Uvaria. The lectotype (Blume s.n., P 00248470) is selected as the best preserved sheet among Blume's original material, containing a full intact flower. Blume, correctly, noted that there are differences between this species and a related one from Timor with larger leaves and more obovate petals, which he called U. timoriensis Blume. Dunal included the two taxa under the same name, as the Paris and Geneva sheets that contain the type specimens for Guatteria rufa (Poivre and Lahaie collections from Java) also include the Timor taxon (Riedlé collections from Timor).

\section{Uvaria Iobbiana Hook.f. \& Thomson, Fl. Ind. 1: 100} (1855)

Type: MALAYSIA. Peninsular Malaysia, Malacca, 1845, $W$. Griffith s.n. (lecto: K 000380689 !, fide I.M.Turner, Phytotaxa 36: 102 (2011)); MALAYSIA. Malacca, s. dat., W. Griffith s.n. (syn: BM!, K 000380688 !, K 000380692 !, P!, TCD 0017973!); s. loc., s. dat., leg. ign., E.Ind.Comp. no. 434, 435 (K!); s. loc., s. dat., T. Lobb s.n. (syn: K 000691177 !, SING!).

Uvaria subrepanda Wall., Numer. List no. 6483 (1832), nom. inval., nom. nud. Uvaria ptychocalyx Miq., Ann. Mus. Bot. Lugduno-Batavi 2: 4 (1865).

Type: INDONESIA. Borneo, Poeloe Lampei, Sakoembang, s. dat., P.W.Korthals s.n. (lecto: L 0195619!, fide I.M.Turner, Phytotaxa 36: 103 (2011)).

Large climber up to $30 \mathrm{~m}$. Young-shoot hairs sparse, manybranched, becoming subglabrous, striate, with prominent lenticels. 
Leaves elliptic-narrowly obovate, base shortly cordate, apex acute-cuspidate, $100-175(-200) \times 40-75(-85) \mathrm{mm}$, coriaceous; veins 8-14; indument upper blade subglabrous, hairs $\sim 20$ branched, midrib subtomentose, lower blade subglabrous, midrib subtomentose. Petiole 3-4 × 1.25-2 mm, appearing much broader than midrib base, sparse to subtomentose, transverse foliations prominent. Inflorescence supra-axillary or rarely leaf-opposed, flowers $1-2(-4)$, peduncle $\sim 2-5 \times$ $1.5 \mathrm{~mm}$, pedicel $5-10(-14) \times 1.5 \mathrm{~mm}$, both usually flattened in transverse section and densely tomentose with large rufous hairs, bracts broadly ovate, $~ 6-10 \times 6-10 \mathrm{~mm}$, membranous, sparse-subtomentose with tomentose margin, veins prominent. Calyx sepals 3 , undulate and imbricate in bud, ovate, $7 \times 3.5 \mathrm{~mm}$ long, recurving at tips, outer- and inner-surface indument denser than on bracts. Corolla 2 subequal whorls of 3 petals, petals yellow but often turning pink or red, inner whorl $\sim 10 \times 8 \mathrm{~mm}$, outer whorl $11 \times 8 \mathrm{~mm}$, shortly and broadly ovate, less often elliptical, apex obtuse or bluntly acute, inner surface undulate, tomentose, outer surface often with transverse ridges of large blunt stellate hairs. Stamens numerous, 2.25-2.5(-3) $\mathrm{mm}$, connective flattened and shortly elongate with prominent papillae, locules extending full length of stamens, outer whorl of staminodes. Carpels numerous, 2.25-2.5(-3) mm, ovary densely tomentose with ring of elongate hairs $\sim 0.25 \mathrm{~mm}$ long beneath stigma, stigma U-shaped, glabrous, ovules $\sim 6-8(-10)$. Fruit monocarps ovoid or globose, $\sim 16-18 \times 20-25 \mathrm{~mm}$, prominently tuberculed, subtomentose, stipe $35-55(-70) \times$ 1.5-2 mm, finely striate, indument sparse; seeds $4-6, \sim 12 \times$ $10 \times 4.5 \mathrm{~mm}$, smooth, raphe prominent but not raised.

\section{Distribution}

A common species found in lowland rainforests recorded once from the Andaman Islands. Distribution is shown in Fig. 14.

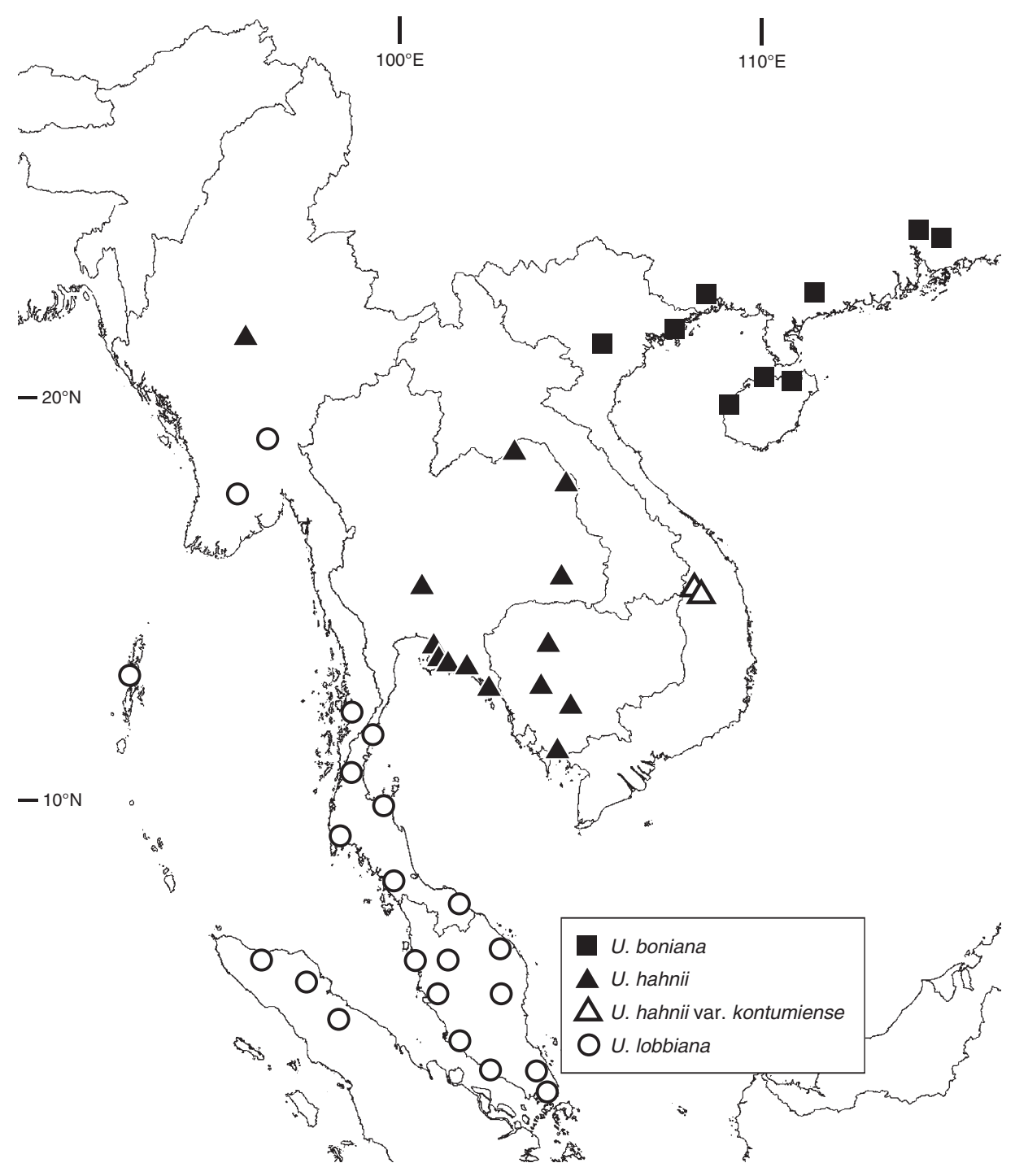

Fig. 14. Distribution of Uvaria boniana, U. hahnii, U. hahnii var. kontumiense and U. lobbiana in South-east Asia on the basis of herbarium specimens verified in this study. 


\section{Notes}

Uvaria lobbiana is morphologically similar to $U$. rufa and $U$. leptopoda (King) R.E.Fr. (lower Malay peninsula). The densely tomentose broadly ovate petals and prominently tuberculate fruits of $U$. lobbiana distinguish it from both these species. The petals of $U$. leptopoda are glabrous on the inner surface, and the fruits, although mounted on a long stipe, are smooth and tomentose. The fruits of $U$. rufa have a short $~ 25-30$ $\mathrm{mm}$-long stipe and $\sim 5-14(-20+)$ seeds, and the stamens are much reduced in number with an outer whorl of 14-20 broadened staminodes. The staminodes of $U$. lobbiana are the same width as the outermost whorl of fertile stamens and 30-40 in number.

\section{Uvaria vietnamensis Meade, Adansonia 27: 17 (2005)}

Type: VIETNAM. Quang Nam, Ba Na, $25 \mathrm{~km}$ north-west of Dà Năng, 24 July 1923, E. Poilane 7326 (holo: P 00248413!; iso: E 00050316!).
[Uvaria fauveliana auct. non Pierre ex Finet \& Gagnep.: S.Jovet-Ast, Suppl. Fl. Indochine 1: 64 (1938), p.p.; S.Jovet-Ast, Not. Syst. 9: 87 (1940), p.p.].

Habit climber or scrambling shrub. Young shoots subtomentose, hairs ferruginous with 2-5 1-mm-long multicellular branches, bark smooth becoming striate and sparse, with occasional persistent larger hairs. Leaves elliptic-narrowly obovate, (70-)90-225 × (35-) 40-75mm, apex acuminate to acute, base narrowly cordate, subcoriaceous; veins $14-16$; indument upper blade sparse-subtomentose, hairs simple, occasionally 2-branched, $\sim 1 \mathrm{~mm}$ long, midrib densely tomentose with erect 1 or $2+$-branched hairs $0.75-1 \mathrm{~mm}$ long; lower midrib and blade subtomentose to sparse with 4-8branched, $\sim 0.6-0.85-\mathrm{mm}$-long stellate hairs. Petiole $3-5 \times$ 1.5-2 $\mathrm{mm}$, indument subtomentose, stellate, hairs fine and pale coloured or more thickly set and ferruginous. Inflorescence not known. Fruit monocarps $\sim 20+$, spheroidal, diameter $\sim 20-30 \mathrm{~mm}$, covered with distinctive 3+-branched

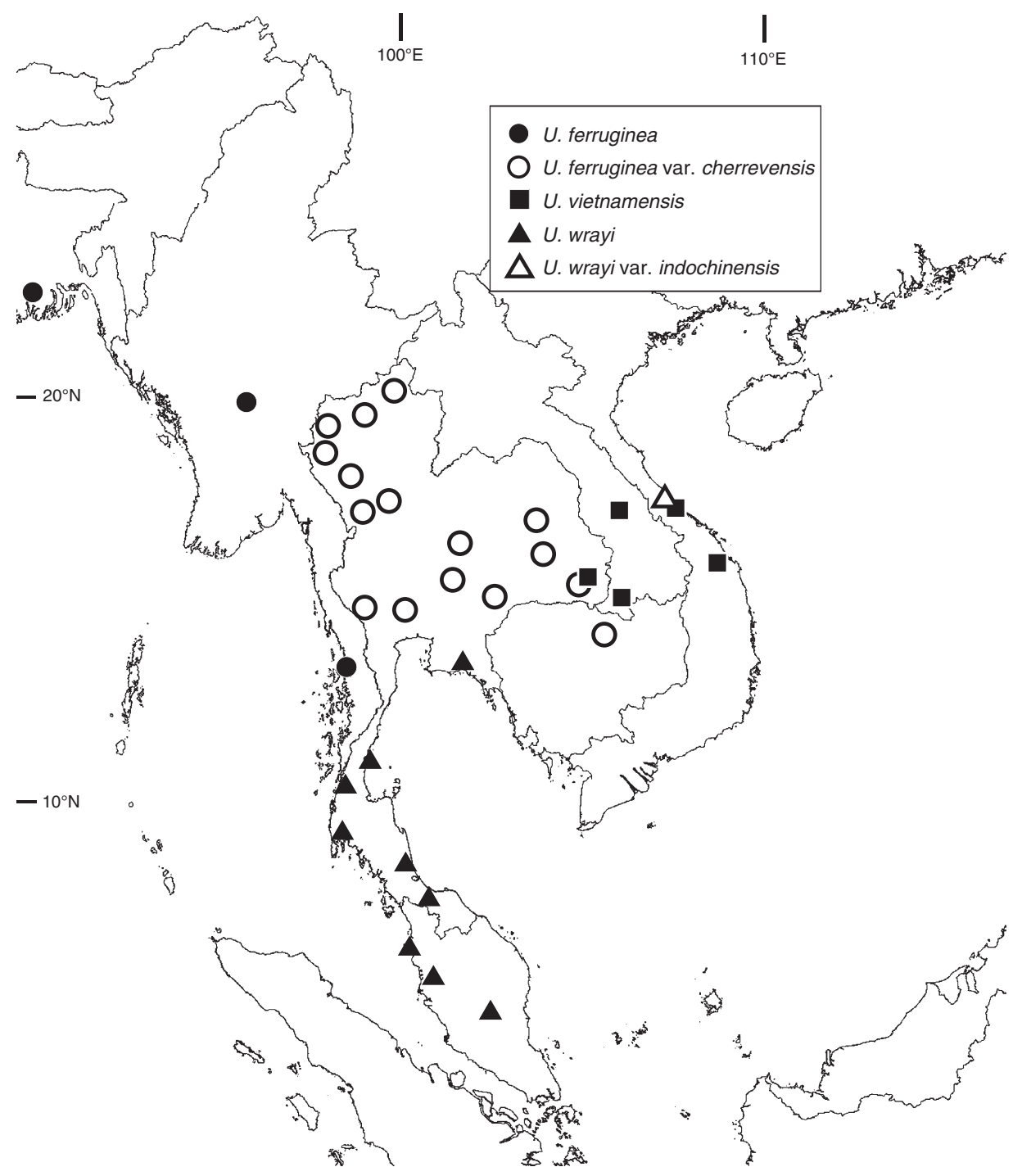

Fig. 15. Distribution of Uvaria vietnamensis, U. wrayi var. wrayi, U. wrayi var. indochinensis, U. ferruginea and $U$. ferruginea var. cherrevensis in South-east Asia on the basis of herbarium specimens verified in this study. 
5-mm-long outgrowths, each covered with ferruginotomentose branches, pericarp red at maturity, flesh pale white, bittersweet to taste, stipes up to $45 \times 2 \mathrm{~mm}$, striate with subtomentose indument; seeds 4-8, shortly hemi-ellipsoidal, $\sim 11 \times 5.25-7 \times 2-3 \mathrm{~mm}$, aril priminent, $4 \times 2.5-3 \times 3 \mathrm{~mm}$, surface leathery, brown, ruminations visible. (Fig. 6.)

\section{Distribution}

A rare species, occurring in mixed-deciduous or dry-evergreen forest habitats above 100-m altitude. Distribution is shown in Fig. 15.

\section{Notes}

Uvaria vietnamensis was described in part from fruiting material collected by Poilane (Poilane 7326) in Vietnam. This material was included by Jovet-Ast $(1938,1940)$ in her concept of $U$. fauveliana (syn. U. hamiltonii var. fauveliana), treated here as a synonym of $U$. rufa. The type material of $U$. hamiltonii var. fauveliana is a Pierre collection from Phu-quoc Island (Pierre 1787) that has flowers but no fruit, and was determined by Meade (2001) to represent $U$. rufa. The bearded epidermis on the fruit of the Poilane 7326 material is unique and clearly distinguishes it from $U$. rufa and all other species in the genus. The flower is unknown.

Uvaria hamiltonii and $U$. hirsuta resemble $U$. vietnamensis in fruit and vegetative morphology respectively. The fruit of $U$. calamistrata has epidermal outgrowths on the monocarp; however, in $U$. vietnamensis these outgrowths are covered with a dense, rufous indument, a feature that is absent in $U$. calamistrata. The stipe in $U$. calamistrata is short and broad, $\sim 3.5 \mathrm{~mm}$ long, whereas it is $\sim 45 \mathrm{~mm}$ long in $U$. vietnamensis. The narrowly obovate leaves of $U$. vietnamensis are also distinct from narrowly elliptic leaves of U. calamistrata (Meade and Parnell 2003).

19. Uvaria griffithii L.L.Zhou, Y.C.F.Su \& R.M.K.Saunders, Syst. Biodivers. 7: 255 (2009)

Cyathostemma viridiflorum Griff., Notul. Pl. Asiat. 4: 707 (1854).

Type: MALAYSIA. Peninsular Malaysia, Malacca, s. dat., W. Griffith 432 (lecto: K 000582099 !, fide T.M.A.Utteridge, Blumea 45(2): 390 (2000)).

Cyathostemma scortechinii King, J. Asiat. Soc. Bengal Part 2, Nat. Hist. 61(1): 9 (1892); Cyathostemma viridiflorum var. scortechinii (King) Ridl., Fl. Malay. Penins. 1: 27 (1922).

Type: MALAYSIA. Peninsular Malaysia, Selangor, Kepong, s. dat., King's collector [H.H.Kunstler] 5857 (lecto: K 000380130!; isolecto: BM 000020753!, SING 0073566!, fide T.M.A.Utteridge, Blumea 45(2): 390 (2000)).

Large climber up to $25 \mathrm{~m}$. Young branchlets sparsely tomentose, quickly becoming subglabrous, bark black and striate. Leaves narrowly obovate, base retuse to cordate, apex acute-shortly acuminate, $100-150 \times 40-60 \mathrm{~mm}$, membranous or coriaceous; veins 9-15; indument hairs erect, subtomentose over upper midrib, quickly becoming sparse-glabrous, glabrous over upper blade, lower surface hairs sparse-subglabrous on midrib, subglabrous-glabrous on blade. Petiole 5-7 mm long $\times$
$1.2-2.5 \mathrm{~mm}$ in diameter, lower side warted, indument sparse, later glabrous. Inflorescence leaf-opposed, sometimes cauliflorous, cymose, dichotomous, flowers 1-4, peduncle elongate, $\sim 20-50 \mathrm{~mm}$ long $\times 1.5 \mathrm{~mm}$ in diameter, pedicel $20-30 \mathrm{~mm}$ long, secondary buds arising in axil of medial bract, bracts ovate, $\sim 5 \times 5 \mathrm{~mm}$, hairs sparse, spreading, fine. Calyx sepals valvate, broadly ovate, $5 \mathrm{~mm}$ wide $\times 3 \mathrm{~mm}$ long, often reflexed at maturity, apex acute, ferrugino-tomentose on inner and outer surfaces. Corolla 2 whorls of 3 petals, inner narrower than outer, petals green or yellow, outer whorl broadly ovate, $\sim 5 \times 5 \mathrm{~mm}$, inner whorl $5 \mathrm{~mm}$ long $\times 4 \mathrm{~mm}$ wide, ovate, base constricted, both whorls concave, thickened basally and with prominent transverse foliations on outer surface, outer surfaces sparsely tomentose, hairs pale, fine, straggling, inner surface shortly and densely tomentose, base subglabrous, hairs pale. Stamens numerous, $\sim 2-2.5 \mathrm{~mm}$ long, apex $\sim 0.5-0.7 \mathrm{~mm}$ long, incurved, papillate, papillae often hair-like, locules extending full length of stamen. Carpels numerous, $\sim 3-4 \mathrm{~mm}$ long, ovaries tomentose, stigma glabrous, elongate, conical, $\sim 1-1.25 \mathrm{~mm}$ long, U-shaped in section, ovules 5 or 6 in 2 rows. Fruit monocarps ovoid-oblong but irregular in shape because of compression of pericarp around seeds, 25-40 mm long $\times$ 10-20 mm wide, pericarp thickened, glabrous, stipe $\sim 20 \mathrm{~mm}$ long, $2.5 \mathrm{~mm}$ in diameter; seeds $4-6$, in 2 rows but irregularly arranged in mature fruit.

\section{Distribution}

Restricted to wetter forests of the Malay Peninsula south of the Isthmus of Kra. Distribution is shown in Fig. 8.

\section{Notes}

The fruit of $U$. griffithii resembles that of $U$. pierrei Finet \& Gagnep.; however, the oblong leaves of this latter species are highly distinctive. The inflorescence of $U$. griffithii is similar in structure to that of $U$. wrayi (King) L.L.Zhou, Y.C.F.Su \& R.M. K.Saunders; however, the pedicel of $U$. griffithii is much longer. Owing to the previous description of $U$. viridiflora Ruiz \& Pav. ex G.Don, Zhou et al. (2009) assigned the epithet griffithii in honour of William Griffith, who originally described the taxon.

20. Uvaria tonkinensis Finet \& Gagnep., Bull. Soc. Bot. France (Mém. 4 (2)) 53: 74 (1906)

Melodorum vietnamense Bân, Bot. Zhurn. (Moscow \& Leningrad) 59: 242 (1974), nom. nov. (non Melodorum tonkinense Finet \& Gagnep. (1906)).

Type: VIETNAM. Tonkin, Ninh Binh, Thinh Chau Mountains, 28 Apr. 1884, H. F. Bon 2566 (first step lecto, fide N.T.Bân, Bot. Zhurn. (Moscow \& Leningrad) 59: 242 (1974)); second step lecto, here designated: P 00142693!; isolecto: P 00142692!, P 00248405!).

Uvaria tonkinensis var. subglabra Finet \& Gagnep., Bull. Soc. Bot. France (Mém. 4 (2)) 53: 74 (1906).

Type: VIETNAM. Tonkin, Mt But-Son, 7 Dec. 1883, H. F. Bon 2295A (lecto, here designated: P 00248048!). 
Cyathostemma longipes Craib, Bull. Misc. Inform. Kew 1925: 8 (1925); Uvaria longipes (Craib) L.L.Zhou, Y.C.F.Su \& R.M.K.Saunders, Syst. Biodivers. 7: 255 (2009).

Type: THAILAND. Ubon Ratchatani Province, $\mathrm{Pu}, 4$ Mar. 1924, Kerr 8607 (holo: K 000595343 !; iso: BM 000020745 !, P!).

Melodorum vietnamense var. calcareum Bân, Bot. Zhurn. (Moscow \& Leningrad) 59: 242 (1974).

Type: VIETNAM. Tonkin. Cho Ganh, June 1923, P. A. Petelot 906 (holo: P 00142619!; iso: A 00039447!, BM!, US 00098808!).

Climber or scrambling shrub. Young shoots sparsely tomentose, bark black, striate. Leaves elliptical-obovate, base retuse, apex acuminate or rarely acute, $\sim 130-170 \times 50-60 \mathrm{~mm}$, thinly coriaceous, waxy; veins 9-11; indument upper surface glabrous in mature leaves, lower surface sparse to glabrous, hairs fine, 3-10-branched, rufous. Petiole 5-7 × $1.5 \mathrm{~mm}$, black, lower surface with prominent transverse foliations, hairs subtomentose-sparse, rufous, fine, straggling. Inflorescence leaf-opposed or subopposed, flowers solitary or rarely 2 or 3 from young shoots, peduncle $3-5 \times 1 \mathrm{~mm}$, pedicel elongate, $35-90 \times 1.5-2 \mathrm{~mm}$, flattened medially, striate, subglabrous, basal bract up to $11 \times 3 \mathrm{~mm}$ with prominent central vein, subtomentose-sparse above and below, medial bract very small, $\sim 1 \times 1 \mathrm{~mm}$, ovate acute, tomentose, both bracts usually wanting. Calyx sepals 3 , valvate, free, opening early, remaining small, $\sim 4.5 \mathrm{~mm}$ wide $\times 2 \mathrm{~mm}$ long, surface warted, outer surface with sparse indument, tomentose at margins, inner surface glabrous. Corolla 2 whorls of 3 petals, inner smaller than outer, petals yellow to green in colour, erect, concave, outer petals $\sim 8-15 \mathrm{~mm}$ wide $\times 9-15 \mathrm{~mm}$ long, broadly ovate, apex bluntly acute-obtuse, base narrowed, inner petals 4-8 $\mathrm{mm}$ wide $\times 6-10 \mathrm{~mm}$ long, narrowly ovate to acute, base of both whorls fleshy, apex and margins membranous, outer petals tomentose on inner- and outer-surface margins and apex, glabrous at base, inner petals tomentose on outer surface, inner surface basally glabrous, apex tomentose, hairs on all petals ferruginous, very short, almost papillate. Stamens numerous, $\sim 2 \times 0.4 \mathrm{~mm}$, apex tomentose, locules extending full length of stamen. Carpels $3 \mathrm{~mm}$ long, stigma elongate, $1 \mathrm{~mm}$ long, glabrous, ovary tomentose, hairs ferruginous, 5-branched, $0.5 \mathrm{~mm}$ in diameter, ovules 2 . Fruit monocarps numerous 15-30, green turning red, transversely ovate-glabrous, up to $\sim 9 \times 7 \mathrm{~mm}$ (1-seeded), 10 $\times 9 \mathrm{~mm}$ (2-seeded), pericarp thin, flaking easily after drying, stipe $\sim 25-40 \times 1.25 \mathrm{~mm}$, finely striate with isolated spreading rufous hairs; seeds 1 or 2 , to $\sim 9 \times 7 \times 4.5 \mathrm{~mm}$, hilum A-shaped, $1.5 \times 1.5 \mathrm{~mm}$.

\section{Distribution}

Distribution is shown in Fig. 16.

\section{Notes}

Leaf characters have been used to distinguish varietal taxa in $U$. tonkinensis and Melodorum vietnamense; however, in both cases, these characteristics are not sufficiently distinctive to warrant varietal status. There is considerable variation in pedicel length between specimens; however, this variation does not appear to be correlated with any other character differences nor with any particular geographic distributions. The lectotypes selected present the best-preserved material from the originally cited sheets.

\section{Uvaria boniana Finet \& Gagnep., Bull. Soc. Bot. France (Mém. 4 (2)) 53: 71 (1906)}

Type: VIETNAM. Nin-thai, Ha-Tay Province, 17 Sep. 1888, H. Bon 3956 (lecto, here designated: P 00247945!; isolecto: G 00226477!, P 00247946!, P00557041!); VIETNAM. 21 May 1889, H.Bon s.n. (syn: P 00247944!); Quang-Yen, Quang Ninh Province, May 1886, Balansa 4185 (syn: K 000691172!, P 00247943!, P 00142652!).

Sarmentose shrub up to $2.5 \mathrm{~m}$ or woody climber up to $20 \mathrm{~m}$, crown a dense network of twining branchlets. Young shoots subglabrous to glabrous, with occasional 1-mm-diameter pale, stellate hairs, bark black, striate, shiny. Leaves elliptic-obovate, apex narrowly acute-acuminate, base cuneate, (50-)80-110 $\times$ (20-)35-40 mm, membranous, both surfaces shiny; veins $\sim 8-10$, bifurcation often at mid-point between midrib and margin; indument leaf blade glabrous on both surfaces, upper midrib with sparse 2-5-branched hairs, lower midrib subglabrous. Petiole 4-5 × $1 \mathrm{~mm}$, glabrous except for sparse hairs along abaxial groove. Inflorescence flowers 1(-2), leaf-opposed; peduncle 2-5 $\mathrm{mm}$; pedicel $\sim 40 \mathrm{~mm}$ long, diameter $\sim 1 \mathrm{~mm}$ at base and apex, $0.5 \mathrm{~mm}$ or less medially, surface black, shiny and striate with scattered pale stellate hairs, bracts small and indistinct, up to $\sim 2 \times 1 \mathrm{~mm}$, ovate, usually wanting. Calyx sepals 3 , valvate, connate at base, broadly ovate-triangular, apex rounded, $\sim 2.5 \times 3.5 \mathrm{~mm}$, reflexed basally, apex remaining incurved, inner and outer surfaces red to green drying black, glabrous centrally with ferrugino-tomentose margins especially prominent on inner surface, often persistent. Corolla 2 whorls of 3 petals, inner narrower than outer, petals red, drying black, thickened centrally and basally, thinner towards margins; outer whorl $\sim 8 \times 7 \mathrm{~mm}, 1.5-2 \mathrm{~mm}$ thick, ovate, rounded apically, concave, inner whorl $\sim 8 \times 5 \mathrm{~mm}, 1-1.5 \mathrm{~mm}$ thick, obovate, apex bluntly acute-rounded, concave, base constricted; both whorls \pm glabrous and leathery on external surface, short stellate indument on margins and towards apex, inner surface with short ferrugino-tomentose indument most dense at apex and margins, glabrous at base, both whorls remaining erect and incurved. Stamens numerous, $\sim 1.5-1.7 \mathrm{~mm}$ long, connective cap flat to discoid to convex, indument dense, blunt, sometimes present on external ridge of connective, locules subequal, basal filament evident, staminodes not present, outermost whorls of stamens reduced in size to $\sim 1.2-1.5 \mathrm{~mm}$, with connective cap extending as ridge on external side. Carpels numerous, $\sim 3 \mathrm{~mm}$ long, \pm erect at centre of gynoecium, incurved towards margin, ovary with dense indument of combed stellate hairs, stigma up to $0.75 \mathrm{~mm}$ long, glabrous, U-shaped in section, lobes narrow, ovules $6-8$ in 2 rows. Fruit monocarps $25-45+$, ovoid, ellipsoid or shortly cylindric, $\sim 15-17(-20) \times 11(-13) \mathrm{mm}, \pm$ basally attached, occasionally with a prominent apicule, green turning red to purple at maturity, drying brown or black, surface somewhat warty with short, scattered, stellate hairs, pericarp $\sim 2 \mathrm{~mm}$ thick, soft and juicy when ripe, stipe $\sim 35-45 \mathrm{~mm}$ long, 


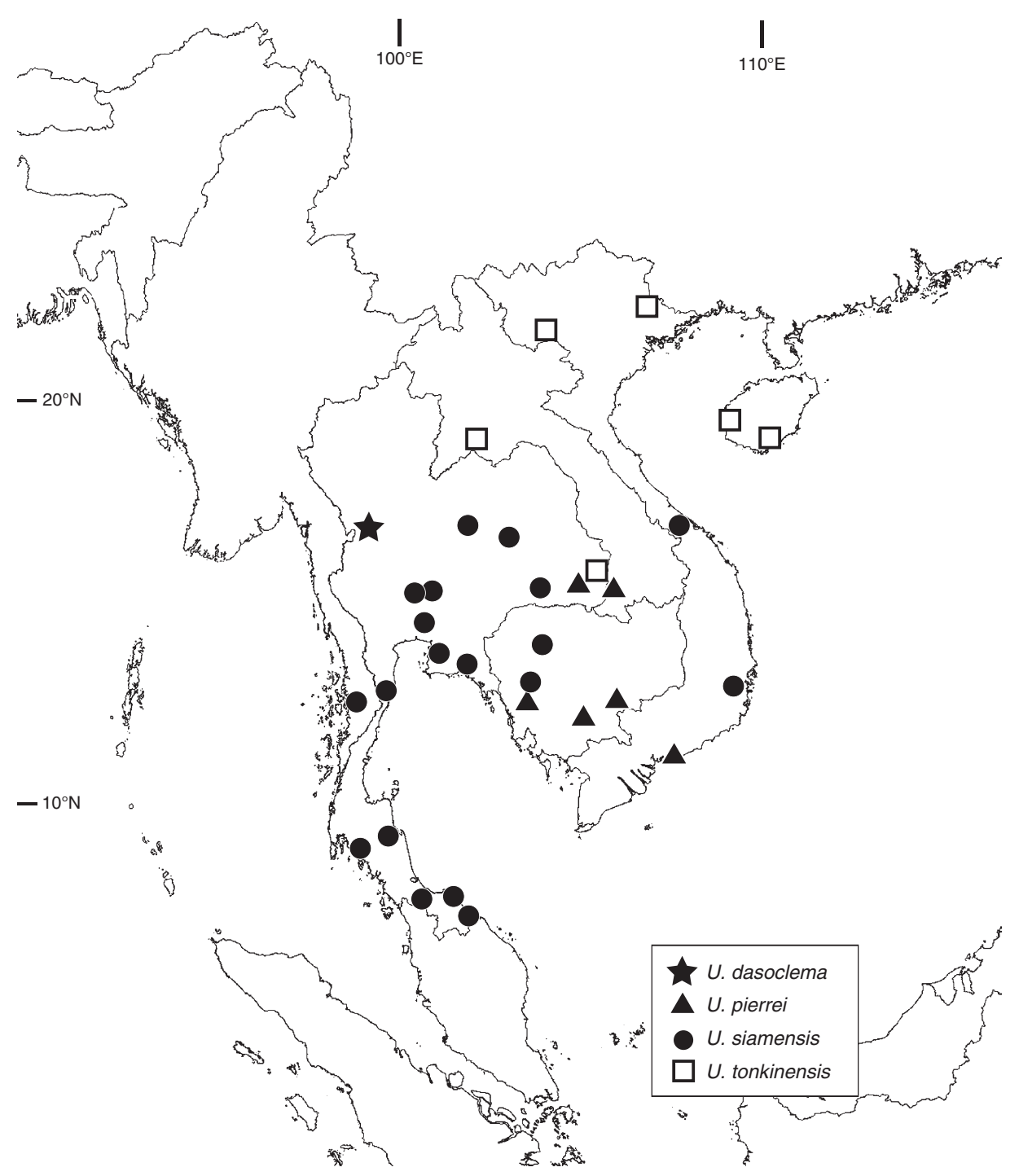

Fig. 16. Distribution of Uvaria dasoclema, U. pierrei, U. siamensis and U. tonkinensis in South-east Asia on the basis of herbarium specimens verified in this study.

$1 \mathrm{~mm}$ in diameter, surface black to brown, striate, indument as for pedicel; seeds $4-6,10 \times 7 \times 4 \mathrm{~mm}$, laterally attached in 2 rows, aril thickened above the raphe on one side, hilum $\mathrm{V}$-shaped, testa brown, shining, ruminations visible. (Fig. 1E.)

\section{Distribution}

Uvaria boniana is typically found in evergreen and semideciduous forest and scrub from sea level to $850 \mathrm{~m}$. In forest habitats, it can grow up to $20 \mathrm{~m}$, and is frequent scrambling over rocks in scrub vegetation on steep open hillsides. Distribution is shown in Fig. 14.

\section{Notes}

The dense twining habit of crown branchlets is distinctive, as are the waxy and almost entirely glabrous leaves. This species is morphologically similar to Uvaria tonkinenis, but distinguished by its much shorter, narrower pedicels, smaller, less broadly ovate petals and smaller, more delicate leaves. Uvaria flexuosa is very similar to $U$. boniana, exhibiting all the floral characters of the latter, but in miniature. The lectotype selected presents the best-preserved material from the originally cited sheets.

22. Uvaria flexuosa Jovet-Ast in F.Gagnepain (ed.), Suppl. FI. Indo-Chine 1: 64 (1938)

Uvaria flexuosa Jovet-Ast, Not. Syst. 9: 73 (1940), isonym.

Type: VIETNAM. Annam, Massif de la Mere et L'Enfant, May 1923, Poilane 6781 (holo: P 00248300!, iso: P 00248301!, PHH!).

Climber with small leaves, crown a dense network of twining branchlets. Young branchlets sparse indument of $\sim 12+$-branched rufous hairs, becoming glabrous, surface black, striate, smooth and shiny. Leaves narrowly elliptical- 
lanceolate, base cuneate, apex narrow, tapering, acuteacuminate, $\sim 70-75 \times 20 \mathrm{~mm}$; veins $\sim 10-13$, branching early; indument upper surface glabrous except for tomentose hairs over midrib and sparse ring of hairs on margin, lower blade glabrous, hairs sparse on midrib. Petiole $2-6 \times 0.75 \mathrm{~mm}$, subtomentose-subglabrous, shiny, black, transversely foliated. Inflorescence flowers solitary, leaf-opposed or supra axillary, peduncle subsessile, $\sim 1-2 \mathrm{~mm}$ long, pedicel $\sim 30 \mathrm{~mm}$ long, very narrow, $\sim 0.35-0.45 \mathrm{~mm}$ in diameter, smooth and shiny, black with sparse rufous hairs as for shoots pedicel with single basal obovate-round $\sim 3 \times 3$-mm bract with hairs on margin and midrib. Calyx sepals 3 , valvate, broadly ovate, $\sim 1 \times 1 \mathrm{~mm}$, tubercled, with sparse spreading rufous hairs, dense at margin. Corolla 2 whorls or 3 petals, inner whorl narrower than outer, outer whorl ovate, $4.5 \mathrm{~mm}$ long $\times 3 \mathrm{~mm}$ wide, inner whorl obovate with narrowed base, $5 \mathrm{~mm}$ long $\times 2.5 \mathrm{~mm}$ wide, inner and outer surface of both whorls basally subglabrous with tomentose margin and apex. Stamens numerous, $\sim 1.2 \mathrm{~mm}$ long, connective apex enlarged, club-shaped, broad, papillate, locules subequal, nearly full length, basal filament evident. Carpels numerous, $\sim 2 \mathrm{~mm}$ long, ovaries hairy on ridges and apex, stigmas glabrous, U-shaped, elongate, ovules 6. Fruit not known.

\section{Notes}

This species is known only from the type collection. There is little discrete character difference between this specimen and $U$. boniana, other than the much smaller size of all organs in U. flexuosa. However, it is very unusual in the Uvaria group to see both young leaves and young flowers growing together on the same new shoots. It must be assumed, therefore, that the smaller leaves of $U$. flexuosa are not a juvenile form, but are distinctive for the species. For this reason, it seems wise to retain $U$. flexuosa until more material becomes available.

\section{Distribution}

Distribution is shown in Fig. 17.

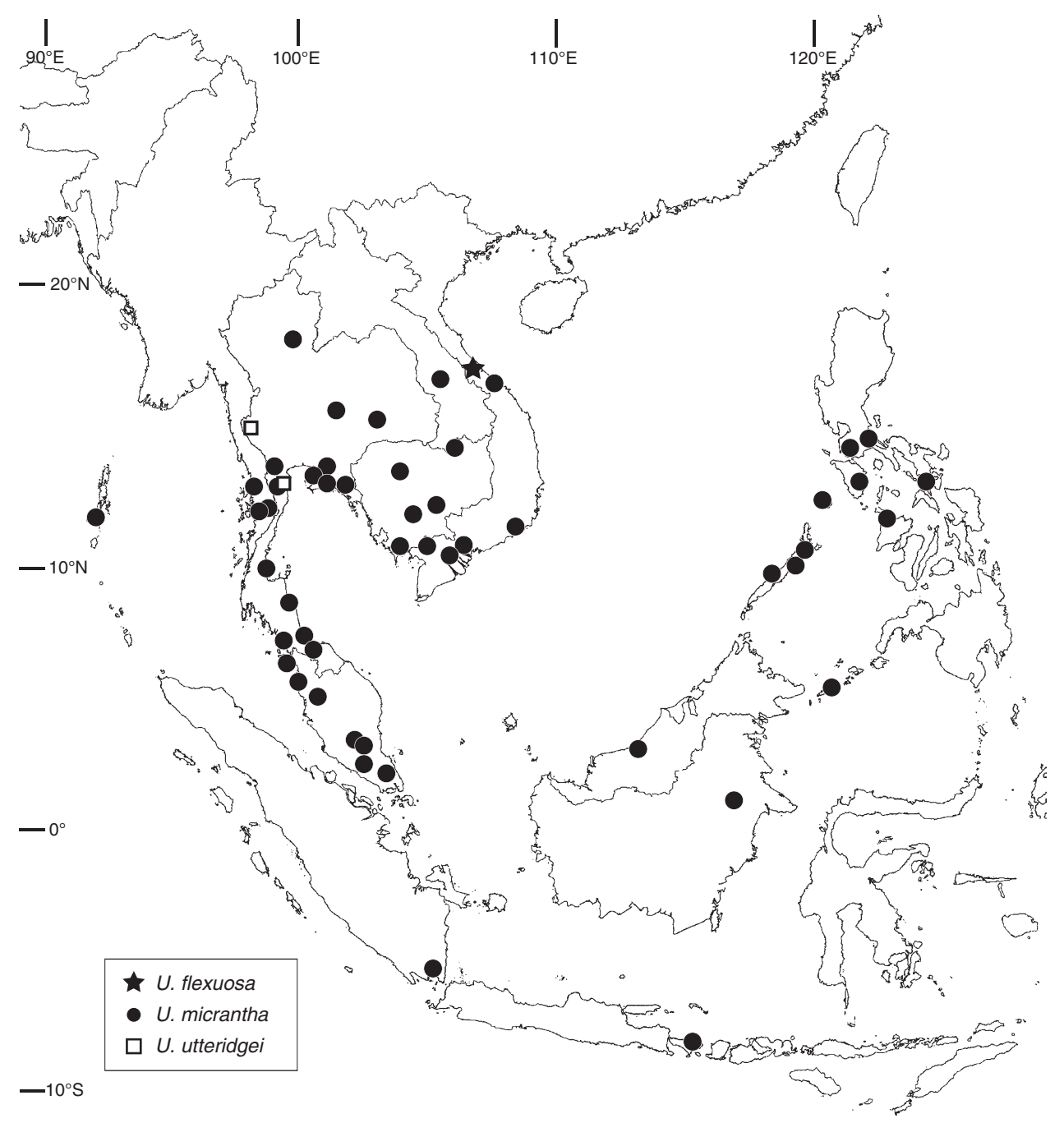

Fig. 17. Distribution of Uvaria flexuosa, U. micrantha and U. utteridgei in South-east Asia on the basis of herbarium specimens verified in this study. 
23. Uvaria pierrei Finet \& Gagnep., Bull. Soc. Bot. France (Mém. 4 (2)) 53: 64 (1906)

Type: CAMBODIA. Pnom-Penh, 29 May 1878, M. Godefroy 48 (lecto, here designated: P 00248308!; isolecto: VNM!, P00248305-7!); CAMBODIA. Pursat, June 1876, Harmand s.n. (syn: P 00248309!); VIETNAM. Baria, Dinhs Mountains, June 1871, Pierre 1789 (syn: A 00039701!, BM 000528084!, PHH!, K 000691170-1!, P 00248310-11!).

Scandent shrub or, more rarely, a climber. Young shoots subtomentose, becoming subglabrous and striate with distinct white lenticels. Leaves distinctively oblong, base retuse, apex obtuse or retuse, $\sim 45-90 \times 17-35 \mathrm{~mm}$, coriaceous, often shiny, leaf margin turned downward; veins $\sim 12-14$; indument upper midrib with sparse erect stellate hairs near base, blade glabrous, lower midrib and veins sparse-subglabrous, blade glabrous. Petiole $\sim 3-4.5 \times 1.25 \mathrm{~mm}$, distinctly broader than midrib, indument sparse. Inflorescence leaf-opposed or subopposed, flowers 2, often with 1 bud dormant, peduncle $\sim 3-4 \mathrm{~mm}$, tomentose-subtomentose, pedicels $\sim 10 \mathrm{~mm}$, often transversely flattened with a longitudinal groove, bracts broadly ovate-acute, $\sim 2.5 \times 4 \mathrm{~mm}$ in diameter, clasping, outer surface subtomentose, inner surface subglabrous. Calyx sepals 3, valvate, opening early, broadly ovate, acute, $\sim 2.25 \times 4.5 \mathrm{~mm}$, sometimes reflexing at maturity, remaining concave, outer surface tomentose at base with thick ferruginous hairs, sparse towards apex, margin tomentose, hairs fine, paler-coloured, inner surface glabrous except for tomentose margin. Corolla 2 whorls of 3 petals, inner whorl marginally smaller than outer, petals purple, narrowly ovate-broadly elliptical, base constricted, apex obtuse or very bluntly acute, $\sim 4.5-5.25 \times 4-4.5 \mathrm{~mm}$, fleshy, thickened basally, both whorls remaining erect, hardly expanding, indument sparse on both surfaces of both whorls, becoming tomentose at margin, hairs fine, spreading. Stamens numerous, $\sim 1 \mathrm{~mm}$ long, connective apex flat, locules extending full length of stamen. Carpels $\sim 40,1.75 \mathrm{~mm}$ long, ridges and neck hairy, stigma large, $\sim 0.6 \times 0.4 \mathrm{~mm}$, broad and elongate, U-shaped, glabrous, with a prominent longitudinal slit, ovules $4-6$ in 2 rows. Fruit monocarps $\sim 5-15$, green maturing yellow then purple, globose or ovoid, $\sim 17.5 \times 12.5 \mathrm{~mm}$, subglabrous, smooth or very finely tuberculate, pericarp fleshy, stipe $\sim 7-12 \mathrm{~mm}$, somewhat striate, sub-glabrous; seeds $2-4$, up to $\sim 12 \times 9 \times 4 \mathrm{~mm}$ at maturity, aligned longitudinally within monocarp. (Fig. 1E.)

\section{Distribution}

Distribution shown in Fig. 16.

\section{Notes}

The peculiar oblong leaves of this species make it instantly recognisable. The only uvariod species in continental Asia the leaves of which resemble it, is Rauwenhoffia siamensis Scheff.; however, this species can be distinguished from $U$. pierrei by its large yellow flowers and subsessile fruits. The longitudinal alignment of seeds in the mature monocarp appears to arise as a result of the non-expansion of tissue between seed attachments after fertilisation, causing the seeds to rotate within the monocarp as they grow. Uvaria goloensis Merr. from the
Philippines is a close relative, and the two are possibly varieties of the same taxon.

\section{Uvaria micrantha (A.DC.) Hook.f. \& Thomson, Fl. Ind. 1: 103 (1855)}

Guatteria micrantha A.DC., Mém. Anon. 42 (1832); Cyathostemma micranthum (A.DC.) J.Sinclair, Gard. Bull. Singapore 14: 225 (1955).

Type: MYANMAR. Martaban, Amherst, 17 June 1827, W. Gomez 15 ['= N.Wallich 1287', Wall. Cat. no. 6449] (lecto: K-W 001123938!; isolecto: BM!, G 00237292!, fide T.M.A.Utteridge, Blumea 45(2): 388 (2000)).

Polyalthia fruticans A.DC., Mém. Soc. Phys. Genève 5: 216 (1832).

Type: MYANMAR. Gomez s.n. [Wall. Cat. no. 6430, Burma, Tavoy 1827, Myanmar] (syn: K-W 001123876-7!, BM!).

Anaxagorea sumatrana Miq., Fl. Ned. Ind., Eerste Bijv. 3: 382 (1861); Uvaria sumatrana (Miq.) Hook.f. \& T.Thomson, Fl. Br. Ind. 1: 51 (1872).

Type: INDONESIA. Sumatra, Lampong. Sumatra austr. in Lampong prope Tega-nennin, Teijsmann s.n. (holo: L 0181487!)

Popowia nitida King, J. Asiat. Soc. Bengal, Pt. 2, Nat. Hist. 61(1): 92 (1893).

Type: INDIA. Andaman Islands, Hobdaypur, 4 July 1891, King s.n. (lecto: K 000739196; isolecto: K 000739197, both $n . v$., fide T.M.A.Utteridge, Blumea 45(2): 388 (2000)).

Climber or scrambling shrub with narrow tangling branches forming dense thicket. Young shoots subtomentose, striations appearing early. Leaves elliptical-shortly lanceolate-narrowly obovate, base cuneate, apex acuminate-acute, $\sim 70-120 \times$ $20-40 \mathrm{~mm}$, thinly coriaceous, waxy, drying dark brown above and below; veins 9-12 (or up to 16 with long acuminum); indument blade glabrous above and below, hairs on upper midrib subtomentose-sparse, hairs erect, 2- or 3-branched, lower midrib with isolated $\sim 1$ - or 2-branched hairs clinging to midrib, always aligned towards leaf apex. Petiole 2-3(-4) $\times$ $0.75 \mathrm{~mm}$, hairs subtomentose, ferruginous. Inflorescence leafopposed, floral buds $1-3$, often only the primary flower opened, the others enclosed by bracts, peduncle subsessile, $\sim 1.5(-2) \mathrm{mm}$ $\times 0.075 \mathrm{~mm}$ in diameter, pedicel $2-4(-5) \times 0.5 \mathrm{~mm}$, basal bract ovate, 4-6 $\times 1-2(-4) \mathrm{mm}$, medial bract smaller, ovate-acute, up to $1.5 \times 2 \mathrm{~mm}$, peduncle and pedicel flattened, ferruginotomentose. Calyx sepals 3 , valvate, broadly ovate-acute, $\sim 2 \times$ $2.75 \mathrm{~mm}$, concave, outer surface and margins tomentose, becoming sparse centrally, hairs pale, inner surface glabrous and warty basally, tomentose towards margin. Corolla 2 whorls of 3 petals, outer petals broader than inner, petals green or yellow to maroon in colour, outer petals ovate-oblong, apex bluntly acute-narrowly obtuse, $\sim 3.25 \times 2.5 \mathrm{~mm}$, inner petals broadly elliptical with distinctly constricted base, $\sim 3.35 \times 2 \mathrm{~mm}$, apex as for outer petals, both whorls thick and fleshy, concave, spreading at maturity to clearly expose stamens and carpels. Stamens numerous, $\sim 0.6-1 \mathrm{~mm}$ long, connective flat, cap-like, papillate, locules full length. Carpels $\sim 20,0.8-1.5 \mathrm{~mm}$ long, straight or bent inward at margin of gynoecium, hairy along ridges and just beneath stigma, hairs fine, many-branched, stigma U-shaped, glabrous, somewhat elongate, ovules 2(-4). Fruit 
monocarps 10-20, green turning yellow, globose-shortly oblong, $\sim 9.5 \times 8 \mathrm{~mm}$ at maturity, pericarp fleshy, becoming thin and black after drying, stipe $\sim 14-20-(-23) \mathrm{mm}$, glabrous, smooth; seeds $1-2(-3), \sim 7 \times 4 \times 4 \mathrm{~mm}$ when in single-seeded monocarp, up to $7 \times 4 \times 2 \mathrm{~mm}$ when 2 - or 3 -seeded, aril $2.5 \times$ $1.75 \mathrm{~mm}$, triangular, hilum A-shaped.

\section{Distribution}

Although there are some records of $U$. micrantha from open dipterocarp forests in central Thailand, this species is usually found in wetter habitats near watercourses or in wet evergreen forests. Distribution is shown in Fig. 17.

\section{Notes}

Sinclair (1955) transferred U. micrantha to Cyathostemma on the basis of its petal aestivation. With the transfer of the type species of Cyathostemma, C. viridiflorum, to Uvaria (see above), this taxon reverts to the classification of Hooker and Thomson (1855)

\section{Uvaria utteridgei L.L.Zhou, Y.C.F.Su \& R.M.K.Saunders,} Syst. Biodivers. 7: 255 (2009)

Cyathostemma siamense Utteridge, Blumea 45: 389 (2000).

Type: THAILAND. Kanchanaburi, Three Pagodas Pass, 14 May 1946, Kostermans 428 (holo: L!; iso: K 000691368!).

Woody climber. Young shoots shortly tomentose, quickly becoming smooth and striate. Leaves elliptical, base subcordate-broadly cuneate, apex acute-acuminate, up to 150 $\times 50 \mathrm{~mm}$, subcoriaceous; veins 8-10; indument upper surface glabrous at maturity, blunt hairs occasionally persistent along midrib depression, lower blade glabrous with isolated hairs alongside midrib, midrib with scattered 2-5-branched hairs oriented towards leaf apex. Petiole $\sim 6 \times 1 \mathrm{~mm}$, smooth, indument sparse to subglabrous, more dense in abaxial groove. Inflorescence cauliflorous, dichasial, peduncles short and closely packed, $\sim 1 \mathrm{~mm}$ long $\times 2 \mathrm{~mm}$ wide, pedicel up to $22 \times$ $1 \mathrm{~mm}$, basal bract triangular, clasping pedicel, $\sim 4 \times 3 \mathrm{~mm}$, medial bract wanting, pedicel flattened with 2 or 3 shallow longitudinal grooves, indument tomentose on bracts, tomentosesubtomentose on peduncle and pedicel, up to 5 mature flowers at a time. Calyx sepals 3, valvate, basally connate, ovate, up to $\sim 7 \mathrm{~mm}$ wide $\times 5 \mathrm{~mm}$ long, subtomentose on both surfaces. Corolla 2 whorls of 3 petals, inner smaller than outer, petals adjoining petals imbricate along entire margin, outer petals ovate, bluntly acute, concave, coriaceous, up to $9 \mathrm{~mm}$ long $\times$ $7 \mathrm{~mm}$ wide, inner whorl narrowly ovate, bluntly acute, concave, coriaceous, up to $8.5 \mathrm{~mm}$ long $\times 6.5 \mathrm{~mm}$ wide, bases of both whorls somewhat thickened, inner and outer indument shortly tomentose. Stamens numerous but reduced to 3 or 4 whorls, $\sim 5 \times$ $1 \mathrm{~mm}$, glabrous, connective with prominent extrorse ridge, apex flattened, bluntly acute, papillate, locules extending full length of stamen. Carpels $\sim 50, \sim 5 \times 1 \mathrm{~mm}$, ovary tomentose, stigma narrow, $1 \mathrm{~mm}$ long, $\mathrm{U}$-shaped, glabrous, ovules $\sim 16$. Fruit unknown. (Fig. 7.)

\section{Distribution}

Uvaria utteridgei is restricted to the dry evergreen forests of south-western Thailand. Distribution is shown in Fig. 17.
Notes

The petals are similar to those of $U$. littoralis and U. microcarpa, and are distinctly imbricate in bud, and the $\sim 16$ ovules reminiscent of Uvaria grandiflora or U. rufa.

26. Uvaria argentea Blume, Fl. Javae $21,22: 24$ \& t. 6 \& t. $13 \mathrm{~d}$ (1830)

Cyathostemma argenteum J.Sinclair, Sarawak Mus. J. 5(3): 599 (1951). Cyathostemma nitidum Bakh.f., Blumea 12: 61 (1963), nom. illeg.

Type: INDONESIA. Java, at Menes, Bantam, June 1823, van Hasselt s.n. (holo: L 1770530!).

\section{Key to the varieties in continental Asia}

1. Petals red, leaves elliptic or oblong...... 26a. U. argentea var. argentea Petals white or yellow to green, leaves usually obovate. 26b. U. argentea var. bracteata

\section{6a. Uvaria argentea Blume var. argentea}

Much-branched climber with distinctive wrapping branchlets. Leaves elliptic or oblong, less often obovate, 50-100(-180) $\times$ $30-50(-65) \mathrm{mm}$, obtuse, acute or shortly acuminate, narrowly retuse, membranous; veins (9)-10-12-(13), fine, gradually inarching, trailing traces fine, not forming distinct submarginal vein, secondary veins kinked or incomplete, tertiary venation very fine, dense, reticulate, midrib shallowly depressed above, emergent below, primary veins marginally raised above, distinct below, microvenation visible above and below; indument upper blade glabrous, sparse stellate erect hairs over midrib, sparse spreading hairs over primary veins, lower surface sparse hairs on midrib and veins, blade glabrous. Petiole 4-7 $\times 1.5 \mathrm{~mm}$, tomentose becoming subglabrous, adaxial surface prominently warted and transversely foliated. Young shoots tomentose becoming subglabrous and prominently striate early. Inflorescence flowers solitary or occasionally 2-4, peduncle $2 \mathrm{~mm}$ long, pedicel up to $7 \mathrm{~mm}$, basal bract $7-9 \mathrm{~mm}$ long $\times 5-6 \mathrm{~mm}$ wide, membranous, medial bract $\sim 5 \times 3.5 \mathrm{~mm}$, obovate, often wrapping over floral bud, indument dense over peduncle, pedicel and bracts, becoming less dense as bracts expand. Calyx sepals 3, free, valvate, broadly ovate, apex obtuse-cuspidate, up to $4.5 \mathrm{~mm}$ wide $\times 3 \mathrm{~mm}$ long, often reflexed at maturity, indument tomentose on both surfaces, outer surface hairs somewhat rufous, on inner surface hairs straggling, fine, often subglabrous basally. Corolla 2 whorls of 3 petals, inner petals smaller than outer, petals red in colour, drying dark green-black, outer petals ovate-oblong, acute, concave, 5-6 mm long $\times 4.5-5.5 \mathrm{~mm}$ wide, inner whorl narrowly ovate, acute, strongly concave, $4-5 \mathrm{~mm}$ long $\times 3.5-4 \mathrm{~mm}$ wide, outer surface of both whorls warty, indument sparse, tomentose at margin, hairs straggling, straw-coloured, inner surface hairs fine, straggling, glabrous medially on outer petals, glabrous basally on inner petals, with prominent stamen indents in both whorls, both whorls remaining erect. Stamens numerous, $2 \mathrm{~mm}$ long, connective with short, blunt tomentum, apex 0 $0.75 \mathrm{~mm}$ long, oblongoidal or globose, locules extending full length of stamen. Carpels $\sim 30,3.5 \mathrm{~mm}$ long, S-shaped, ovaries hairy, stigma U-shaped, elongate, 1-1.25 mm long, glabrous, 
ovules 4-10. Fruit monocarps numerous, red or purple, ovoid, $\sim 10-18 \times 10-12 \mathrm{~mm}$, glabrous, stipe $\sim 10-15 \mathrm{~mm}$ long, glabrous; seeds $1-2, \sim 9 \times 7 \times 4 \mathrm{~mm}$.

\section{Distribution}

In continental Asia restricted to peninsular Malaysia. Distribution is shown in Fig. 11.

\section{Notes}

Uvaria argentea var. argentea is recognised from herbarium specimens by its membranous leaves and grey-green to black petals with a pale tomentose indument on the margins.

26b. Uvaria argentea var. bracteata (Roxb.) Meade \& J.Parn., comb. et. stat. nov.

Uvaria bracteata Roxb., Fl. Ind. Ed. 1832 2: 660 (1832).

Type: 'Uvaria bracteata', Roxb. Icon. 2290 (lecto: K!, fide T.M.A.Utteridge, Blumea 45(2): 383 (2000)).

Uvaria bracteata Roxb., Hort. Bengal. 43 (1814), nom. inval., nom. nud. Uvaria bracteata Wall., Numer. List no. 6468 (1832), nom. inval., nom. nud.

Uvaria gomeziana A.DC., Mém. Soc. Phys. Genève 5: 203 (1832).

Type: MYANMAR. Tavoy, 8 Sep. 1827, W.Gomez 197 [Wall. Cat. no. 6459] (lecto: K-W 001123956!, fide I.M. Turner, Phytotaxa 36: 47 (2011)).

Cyathostemma yunnanense Hu, Bull. Fan Mem. Inst. Biol. 10: 121 (1940).

Type: CHINA. Yunnan, Fo Hai, Wang 74547 (holo: PE 00028347!; iso: PE 00028346!, A 00039271!).

Differs from the type variety in having white or yellow to green petals, and the leaves are usually larger and almost exclusively obovate.

\section{Distribution}

Uvaria argentea var. bracteata occurs in mountain foothills up to $\sim 1100 \mathrm{~m}$. Distribution is shown in Fig. 11 .

\section{Notes}

Sinclair (1951) treated $U$. argentea var. bracteata as synonymous with Cyathostemma argenteum (=Uvaria argentea). However, the petals of $U$. argentea var. bracteata are always green or yellow or grey or white, as opposed to deep red or purple for $U$. argentea var. argentea, and there also is a marked disjunction in the distributions of the two taxa. For these reasons, it seems wise to retain the two as varieties. The type of $C$. yunnanense matches the Roxburgh lectotype of U. bracteata. Roxburgh (1832, p. 661) described the fruits of this species as '...few, pendulous, of the size of a small pullet's egg, from oval to oblong, obtuse at both ends, smooth, when ripe of a rich yellow. Seeds a few, oval, compressed, smooth...'. On the basis of the accounts of Sealy (1956) and Forman (1997), a Roxburgh specimen at BM(!) labelled as ' $U$. bracteata' is probably the only surviving material on which he based his Icones illustration; however, this material is not listed in the lectotypificaion of Utteridge (2000).

27. Uvaria wrayi (King) L.L.Zhou, Y.C.F.Su \& R.M.K. Saunders, Syst. Biodivers. 7: 255 (2009)

Cyathostemma wrayi King, J. Asiat. Soc. Bengal Pt. 2, Nat. Hist. 61(1): 9 (1892).

Type: MALAYSIA. Perak, s. dat., Scortechini $131 b$ (lecto: K 000691375!, fide T.M.A.Utteridge, Blumea 45(2): 391 (2000)).

\section{Notes}

The large obovate membranous leaves of this species make it easily recognised from sterile specimens. Utteridge (2000) cited the collection number of the lectotype specimen as ' 1316 ', in error for ' $131 b$ ', as corrected above.

\section{Key to the varieties in continental Asia}

1. Leaves obovate or rarely oblong, veins $12-15$. 27a. $U$. wrayi var. wrayi Leaves oblong or rarely narrowly obovate, veins $15-17$ 27b. $U$. wrayi var. indochinensis

27a. Uvaria wrayi (King) L.L.Zhou, Y.C.F.Su \& R.M.K. Saunders var. wrayi

Small shrub or climber. Young shoots sparsely tomentose, distinctive light-ferruginous hue of fine hairs over black bark, hairs persistant, becoming short. Leaves obovate, less often oblong, base retuse, apex acute-acuminate, up to $250 \times$ $95 \mathrm{~mm}$, membranous, veins 12-15; indument upper-surface hairs erect and shortly tomentose over midrib, spreading, sparseglabrous over veins, blade subglabrous-glabrous; lower blade subglabrous with occasional hairs on tertiary venation, midrib hairs sparse, spreading. Petiole indument ferrugino-tomentose, later sparse, hairs fine, straggling. Inflorescence axillary or supra-axillary, often cauliflorous, flowers $2-5$, peduncle up to $3 \mathrm{~mm}$, pedicels $2-8 \mathrm{~mm}$ long, bracts laminar, acute, enlarging up to $4 \times 2 \mathrm{~mm}$, narrowly ovate-acute, ferrugino-tomentose, later sparse. Calyx sepals 3, free, opening early and reflexing, remaining small, concave, broadly ovate-acute, coriaceous, up to $4 \mathrm{~mm}$ wide $\times 3 \mathrm{~mm}$ long, indument subtomentose, hairs fine, ferruginous. Corolla 2 subequal whorls of 3 petals, petals green to yellow in colour, free, imbricate at tips, broadly ovate in bud, becoming narrowly ovate, bluntly acute-obtuse, thickly coriaceous-fleshy, up to $\sim 9 \mathrm{~mm}$ long $\times 6 \mathrm{~mm}$ wide, often opening but not reflexing, indument subtomentose-sparse, hairs becoming short, blunt, almost papillate, glabrous basally on inner side. Stamens numerous, $\sim 1.4 \mathrm{~mm}$ long, glabrous, connective flattened, distinctly papillate, locules extending full length of stamen. Carpels numerous, $\sim 2 \mathrm{~mm}$ long, neck of longer hairs at apex of ovary, below glabrous except on ridges, arched inwards at outer whorls, stigma glabrous, elongate, ovules 2 or 3, lateral. Fruit monocarps numerous, ovoid, $\sim 9-13 \times 6-9 \mathrm{~mm}$, stipe 15-20 mm, narrow, pericarp thin, flaking off easily when dry, thickened apicule often present, seeds 1 or $2, \sim 9 \times 8 \times 4 \mathrm{~mm}$, single seeds ellipsoidal or proncouncedly hemi-ellipsoidal when 2 seeds present, hilum A-shaped, $\sim 1.5 \times 1.5 \mathrm{~mm}$. 


\section{Distribution}

Uvaria wrayi var. wrayi is common across its range, occurring in evergreen forests up to $\sim 400 \mathrm{~m}$. Distribution is shown in Fig. 15.

27b. Uvaria wrayi var. indochinensis (Jovet-Ast) Meade \& J.Parn., comb. nov.

Cyathostemma wrayi var. indochinensis Jovet-Ast., Notul. Syst. 9: 86 (1940).

Type: VIETNAM. Annam, Blao, Haut Donai Province, 13 Feb. 1933, Poilane 21930 (lecto, here designated: P 00411099!; isolecto: P 00411100!, P 00411101!); VIETNAM. Annam, Blao, Haut Donai Province, 26 May 1933, Poilane 22517 (Syn: P 00234100!, P 00411102-3!)

Jovet-Ast described this variety on the basis of the greater number of leaf veins, plus the distinctly oblong as opposed to obovate leaves, compared with typical Cyathostemma (Uvaria) wrayi, a difference that is evident in the composite leaf shape for $U$. wrayi var. wrayi as presented in Meade and Parnell (2002, p. 242). Poilane 15387 from north-western Cambodia, cited as type material by Jovet-Ast, is in fact $U$. wrayi var. wrayi. The lectotype selected presents the best-preserved material from the originally cited sheets.

\section{Distribution}

Uvaria wrayi var. indochinensis is known from one locality, in Vietnam. Distribution is shown in Fig. 15.

\section{Uvaria hahnii group}

28. Uvaria dasoclema L.L.Zhou, Y.C.F.Su \& R.M.K. Saunders, Bot. J. Linn. Soc. 163: 37 (2010)

Monocarpia siamensis Craib, Bull. Misc. Inform. Kew 1924: 81 (1924); Dasoclema siamensis (Craib) J.Sinclair, Gard. Bull. Singapore 14: 273 (1955).

Type: THAILAND. Kampeng Pet, Me Lamung, 8 June 1932, Kerr 6087 (lecto, here designated: K00691507!; isolecto: K 00691506!).

Scrambling shrub or climber. Young shoots sparsely tomentose. Leaves elliptical-oblong, apex acute-shortly acuminate, base retuse or rarely cuneate in younger leaves, up to $125 \times 60 \mathrm{~mm}$, membraneous, veins 6-13, indument upper surface sparse becoming glabrous, midrib hairs erect, subtomentose becoming sparse, lower surface hairs sparse on midrib and veins, blade sparse-subglabrous. Petiole 5-8 $\mathrm{mm}$, sparsely tomentose. Inflorescence solitary, leaf-opposed, pedicel up to $60 \mathrm{~mm}$, diameter 1-3 mm, widest at base of receptacle, sparsely tomentose, bracts wanting. Calyx sepals 3, valvate, ovate-acute, up to $5 \times 6 \mathrm{~mm}$, outer surface tomentose, inner surface basally glabrous. Corolla, 2 whorls of 3 petals, inner petals smaller than outer, petals white to green in colour, outer whorl $25-30 \mathrm{~mm}$ long, 8-10 mm wide, free, inner whorl 23-28 $\mathrm{mm}$ long, 5-8 $\mathrm{mm}$ wide, lower margins often connivent, outer surface sparsely tomentose, inner surface mostly glabrous, margins and apex sparsely tomentose. Stamens numerous, $2.5 \mathrm{~mm}$, cuneate, locules raised on short filament, connective apex convex or flattened, smooth. Carpels solitary, ovules 6-12, laterally inserted in 2 rows, stigma disc-shaped, $3 \mathrm{~mm}$ in diameter, glabrous. Fruit monocarp solitary, subsessile, ovoid-oblong, $15-35 \times 10-15 \mathrm{~mm}$; stipe subsessile, 3-4 × 3-4 mm, tomentose; seeds 6-12.

\section{Distribution}

A rare species, restricted to limestone areas in central Thailand. Distribution shown in Fig. 16.

\section{Notes}

The unusual single carpel and broad flat stigma of this taxon are understood now to be derived characteristics that have caused much taxonomic confusion (Zhou et al. 2010). All other morphological traits are characteristic of Uvaria; the raised stamen locules and subequal petal whorls reflect its phylogenetic affinity with Hahnii-group taxa. The lectotype selected presents the best-preserved material from the originally cited sheets.

\section{Uvaria ferruginea Buch.-Ham. ex Hook.f. \& Thomson, Fl. Ind. 1: 96 (1855)}

Ellipeia ferruginea (Buch.-Ham. ex Hook.f \& Thomson) Hook.f \& Thomson, Fl. Brit. Ind. 1: 52 (1872); Ellipeiopsis ferruginea (Buch.Ham. ex Hook.f \& Thomson) R.E.Fr., Ark. Bot. ser. 2, 3: 41 (1955).

Type: MYANMAR. Ava (Tenasserim), Meaday, s. dat., Buchanan-Hamilton s.n. (lecto: BM 000547032!, fide I.M. Turner, Nordic J. Bot. 33 : 290 (2015)).

\section{Notes}

Uvaria ferruginea is of unusual growth habit and morphology. The climbing stem has become modified so that plants may creep directly over the soil, with successive branchlets growing upward. Fries (1955) established the genus Ellipeiopsis and maintained the distinction between var. ferruginea and var. cherrevensis at species level. The key morphological difference between the two is in leaf shape, with var. ferruginea having ovate-oblong leaves and var. cherrevensis having obovate leaves (Meade and Parnell 2003). The two varieties have distinct distributions, with var. cherrevensis occurring in Thailand and Indochina, and var. ferruginea occurring only to the east of the Thai-Myanmar mountain border. The Paris type material of var. cherrevensis is the most complete of the duplicate set and is here designated as the lectotype.

\section{Key to the varieties in continental Asia}

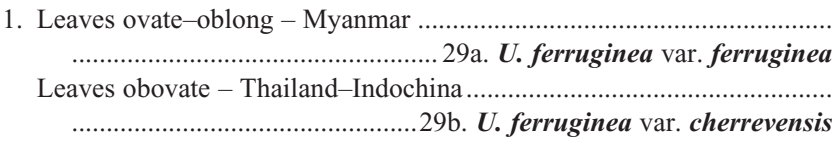

29a. Uvaria ferruginea Buch.-Ham. ex Hook.f. \& Thomson var. ferruginea

A small shrub with a single or branched horizontal stem producing erect $\sim 1-\mathrm{m}$ high branchlets. Young shoots densely ferrugino-tomentose, later striate, sparse-subglabrous. Leaves coriaceous, broadly elliptical-oblong, base cordate, apex obtuse, cuspidate or acute, $120-150 \times 50-80 \mathrm{~mm}$, veins $11-12(-14)$; indument upper blade subtomentose, hairs erect, 1-3-branched, 
pale, becoming sparse-subglabrous with age, midrib and primary vein bases tomentose, hairs erect, $\sim 2-5$-branched; lower blade hairs subtomentose, 6-10+-branched, spreading, rufous, tomentose over midrib and vein traces. Petiole $\sim 8-10 \times$ $2 \mathrm{~mm}$, ferrugino-tomentose. Inflorescence leaf-opposed, flowers solitary, peduncle much reduced, pedicel $\sim 8-12(-20) \times 1 \mathrm{~mm}$, tomentose, medial bract narrowly elliptical, $8-15 \times 2-3 \mathrm{~mm}$, tomentose on both surfaces, venation not prominent. Calyx sepals 3 , basally connate, tips valvate, ovate-acute, $\sim 5-6 \times$ $8 \mathrm{~mm}$, concave, recurving basally at maturity, outer surface densely tomentose, inner surface warty and sparse basally, tomentose elsewhere. Corolla 2 whorls of 3 petals, inner narrower than outer, petals yellow to white in colour, outer petals broadly ovate, $\sim 12-14 \times 10-12 \mathrm{~mm}$, apex bluntly acute, base constricted to $\sim 3.25 \mathrm{~mm}$ wide, outer surface tomentose, inner surface tomentose along margins and at apex, glabrous at base, inner petals narrowly ovate, $\sim 12-14 \times$ 7-8 mm, outer surface tomentose, inner surface basally glabrous, base $\sim 2.25-2.5 \mathrm{~mm}$ wide, with 2 conspicuous marginal glands contiguous to glabrous base of inner surface. Stamens numerous, $\sim 1.8-2 \mathrm{~mm}$, cuneate, connective apex glabrous, rusty in colour, shiny, often extending as ridge down external side, locules raised on short filament. Carpels $\sim 25-30,2.5-3 \mathrm{~mm}$ long, ovary tomentose, hairs fine, many-branched, forming even surface, stigma elongate, $\sim 0.8-1 \mathrm{~mm}$ long, hairy, U-shaped with lateral groove, ovule 1, placentation apical or subapical. Fruit monocarps 5-20, 10 $\times 4.5 \mathrm{~mm}$, red in colour with conspicuous black apicule, pericarp fleshy, smooth, sparse-subglabrous, becoming black, thin and undulate on drying, 1 seed, basally attached, ellipsoidal.

\section{Distribution}

Bangladesh and Myanmar. Distribution is shown in Fig. 15.

\section{9b. Uvaria ferruginea var. cherrevensis (Pierre ex Finet \& Gagnep.) Meade \& J.Parn., comb. et stat. nov.}

Ellipeia cherrevensis Pierre ex Finet \& Gagnep., Bull. Soc. Bot. France (Mém. 4 (2)) 53: 76 (1906); Ellipeiopsis cherrevensis (Pierre ex Finet \& Gagnep.) R.E.Fr., Ark. Bot. ser. 2, 3: 41 (1955); Uvaria cherrevensis (Pierre ex Finet \& Gagnep.) L.L.Zhou, Y.C.F.Su \& R.M.K.Saunders, Syst. Biodivers. 9: 255 (2009).

Type: THAILAND. Petchaburi, Aug. 1868, Pierre 1790 (lecto, here designated: P 00432161!; isolecto: P 00432162!); CAMBODIA. Samrong-Tong, Chereer Mountains, Apr. 1870, Pierre 1790A (syn: A!, C!, L!, NY!, P 00432162-4!); CAMBODIA. Samrong-Tong. Cherreo Mountains, Apr. 1870, Pierre 745 (syn: P 00432165-6!).

Leaves broadly obovate.

\section{Distribution}

Thailand and Cambodia. Although widespread, this taxon is much less common in the evergreen vegetation favoured by Uvaria species, and is unrecorded from most of the evergreen forests in southern Indochina and peninsular and south-eastern Thailand and from from the driest regions of north-eastern Thailand. Distribution is shown in Fig. 15.
30. Uvaria pauciovulata Hook.f. \& Thomson, Fl. Brit. Ind. 1: 51 (1872)

Type: MALAYSIA. Malacca, 14 June 1865, Maingay 104/1023 (lecto, here designated: K000380686!); MALAYSIA. Malacca, 27 May 1868, Maingay 104/3392 (syn: K000380687!, SING!).

\section{Notes}

The inflorescence structure, petals, stamens, carpels and fruits of $U$. pauciovulata are most similar to those of $U$. cuneifolia (Malesia south of the Isthmus of Kra). The major difference between it and $U$. cuneifolia is that the inner petals remain closed and imbricate over the gynoecium in U. cuneifolia, whereas they open in $U$. pauciovulata. The shape, indument and venation of the leaves in the two species are also different. The type material for var. scortechinii shows a variant leaf form compared with the type for $U$. pauciovulata, but otherwise there are no discrete character differences that warrant a species-level distinction.

\section{Key to the varieties in continental Asia}

1. Leaves ovate to elliptic to narrowly obovate with ferruginous leaf venation on abaxial surface .............30a. U. pauciovulata var. pauciovulata Leaves broadly ovate-elliptical with prominent thickened ferruginous leaf venation on abaxial surface ......

30b. U. pauciovulata var. scortechinii

\section{0a. Uvaria pauciovulata Hook.f. \& Thomson var. pauciovulata}

A scandent shrub or woody climber. Young shoots tomentose, hairs ferruginous, stout, 20+-branched, quickly becoming sparse, bark black, striate. Leaves (ovate-)elliptic-narrowly obovate, base shortly cordate or retuse, apex acute, 95-140 $\times$ 50-60 mm, coriaceaous; veins 9-12(-14), almost perpendicular to midrib at base, then arching gradually; indument hairs manybranched, ferruginous, young leaves with sparse hairs on both surfaces, at maturity upper surface glabrous, lower blade subglabrous, hairs tomentose-sparse on vein bases and midrib. Petiole $\sim 4-5 \times 1.5 \mathrm{~mm}$, hairs subtomentose-sparse, verrucose. Inflorescence terminal, cymulose, flowers 1 or 2, rarely 3, peduncle $2-7 \mathrm{~mm}$ long, pedicel $\sim 8-10 \times 2.5 \mathrm{~mm}$, broad and flattened, tomentose-subtomentose, hairs stocky, $\sim 20+$-branched, ferruginous, bracts ovate, clasping, often enclosing floral buds, persistent, $\sim 9 \times 5 \mathrm{~mm}$, prominent central vein often visible, indument as for pedicel. Calyx sepals 3, basally connate, ovate-triangular, acute, 7-8 × 6-7 mm, somewhat thickened, often persistent, outer surface ferrugino-tomentose as for pedicel and bracts, inner surface glabrous basally, surface black, shiny, warted, elsewhere subtomentose, hairs 1-5branched, downy, combed. Corolla 2 whorls of 3 petals, inner narrower than outer, petals white, yellow or cream in colour, large and showy, incurved, coriaceous, outer whorl broadly obovate, obtuse, $\sim 15-20 \times 12-14 \mathrm{~mm}$, base $3.5 \mathrm{~mm}$, coriaceous, undulating, inner whorl narrowly obovate, obtuse, $\sim 15-20 \times 11-13 \mathrm{~mm}$, base $\sim 2.5 \mathrm{~mm}$, indument on outer surface of both whorls as for pedicel and bracts, central vein trace often covered with ferruginous hairs, inner surface of outer whorl subtomentose, hairs downy, straggling, 3-8-branched, petal base glabrous, black, inner surface of inner whorl 


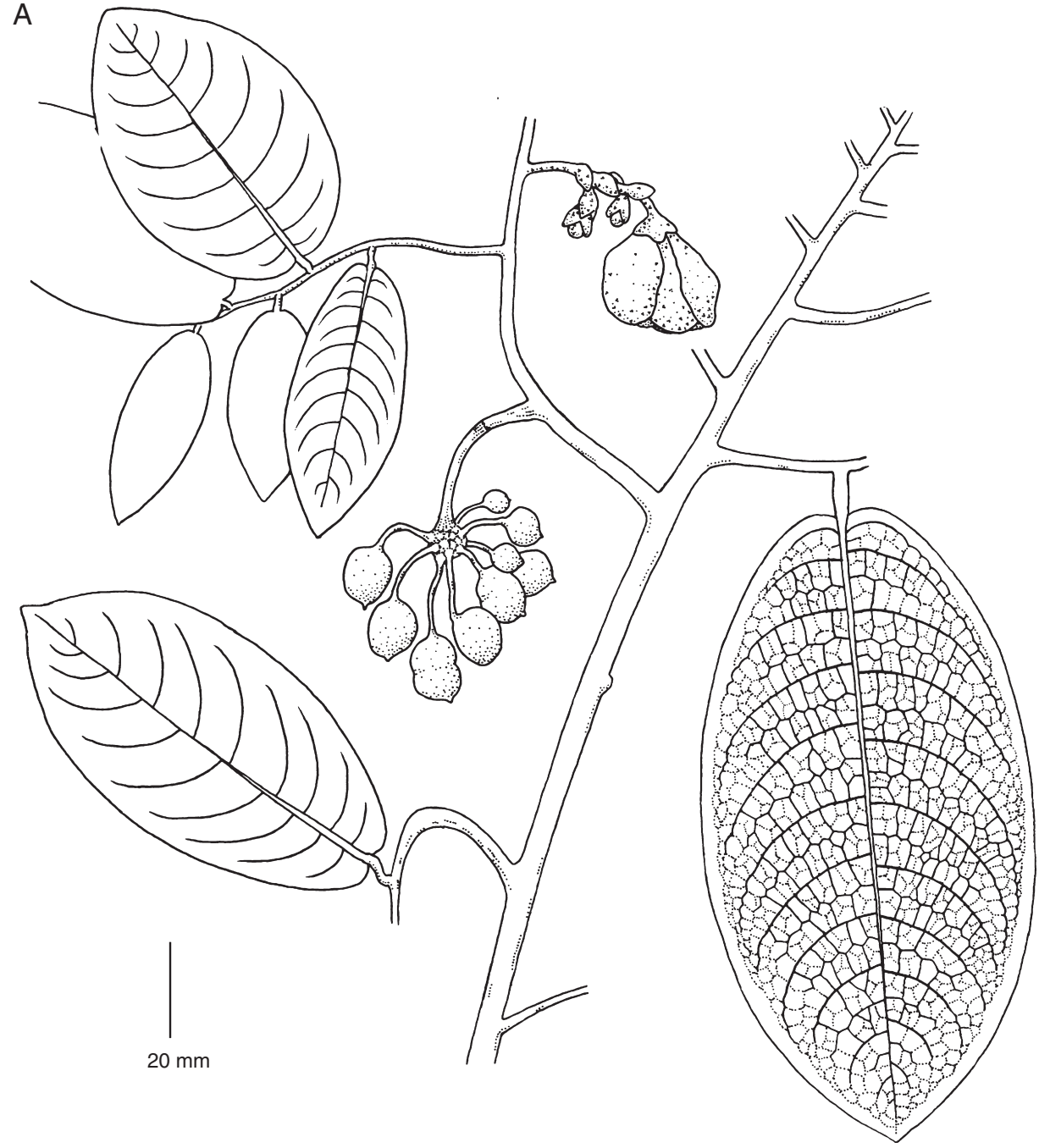

$\mathrm{B}$

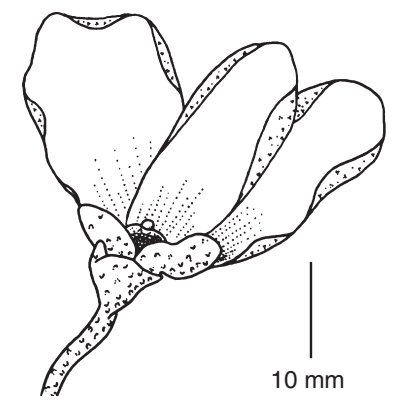

C

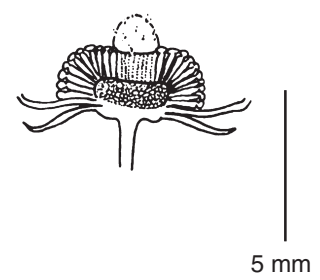

D

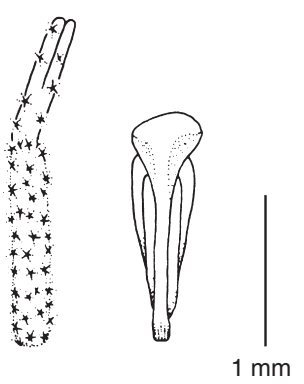

Fig. 18. Uvaria pauciovulata var. pauciovulata. A. Habit. B. Flower, section. C. Flower, section. D. Carpel. E. Stamen. Illustration C. V. Meade. 


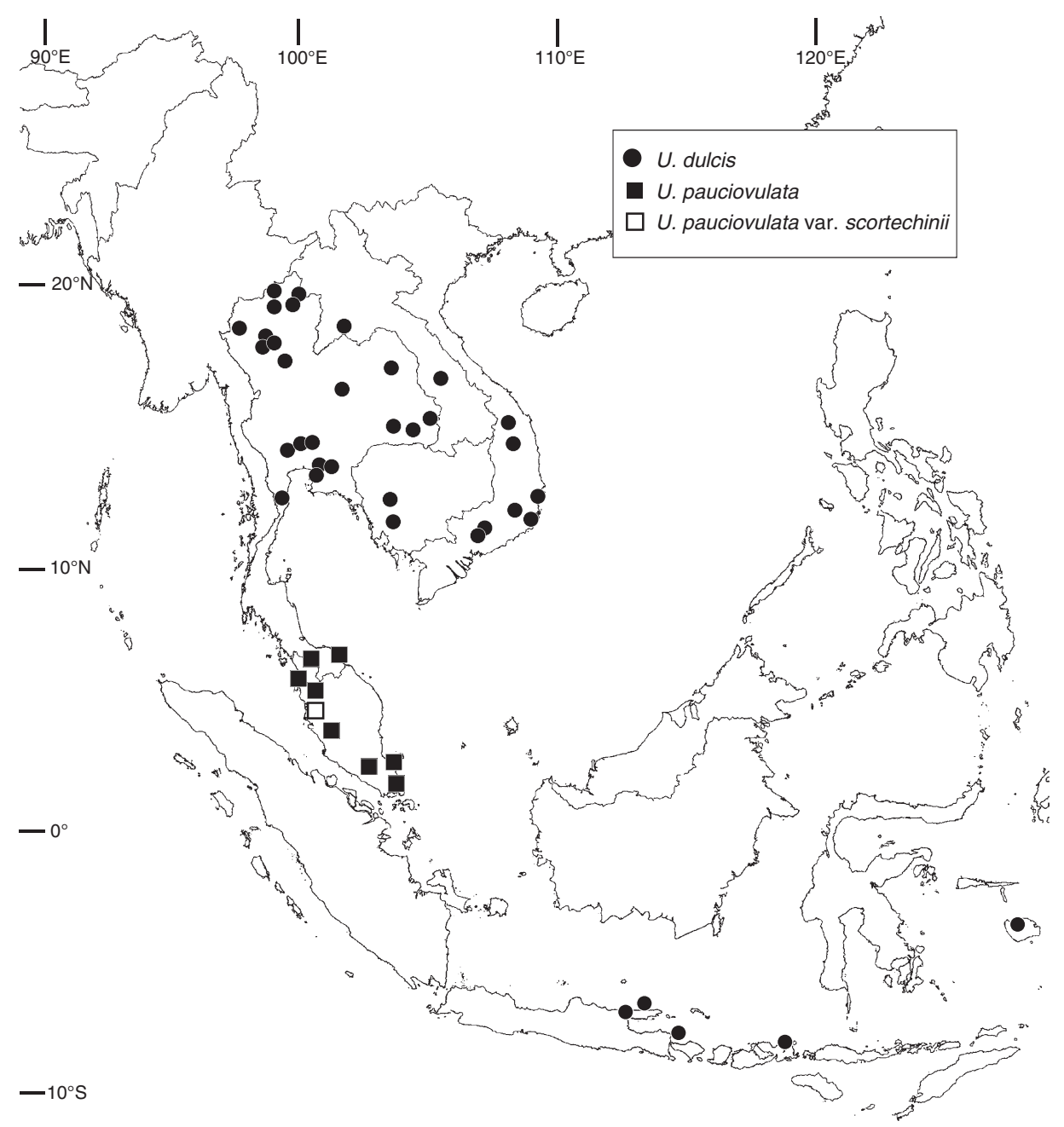

Fig. 19. Distribution of Uvaria dulcis, U. pauciovulata and U. pauciovulata var. scortechinii in South-east Asia on the basis of herbarium specimens verified in this study.

glabrous, except for downy indument at apex and around margins, both whorls remaining incurved. Stamens numerous, $1.25-1.5 \mathrm{~mm}$, cuneate, locules uneven in length, raised on short filament, connective apex convex or flattened, smooth. Carpels numerous, $\sim 2.5-2.75 \mathrm{~mm}$, ovaries tomentose, hairs flattened and spreading, stigmas elongate with sparse stellate hairs over surface, $\sim 1 \mathrm{~mm}$ long, U-shaped with prominent longitudinal slit, ovules 1-2(-3), gynoecium dome-shaped, raised. Fruit monocarps numerous, spheroidal or shortly oblongoidal, $\sim 8-23 \times 9 \mathrm{~mm}$ in diameter, pericarp thin with prominent apicule, monocarps basally or sub-basally attached, stipe $\sim 10-15 \mathrm{~mm} \times 1 \mathrm{~mm}$, striate, monocarp and stipe densely and shortly tomentose; seeds 1 or 2 or very rarely 3, smooth, wrapped tightly by monocarp tissue, ruminations not visible beneath surface, raphe dark, prominent. (Fig. 1D, E, 18.)

\section{Distribution}

Lower peninsular Thailand and peninsular Malaysia. Distribution is shown in Fig. 19. 30b. Uvaria pauciovulata var. scortechinii (King) Meade \& J.Parn., comb. et stat. nov.

Uvaria scortechinii King, J. Asiat. Soc. Bengal, Pt. 2, Nat. Hist. 61(1): 20 (1892).

Type: MALAYSIA. Perak, s. dat., Scortechini s.n. (holo: K 000380695!, iso: A 00039706!).

Leaves broadly ovate-elliptical with prominent, thickened venation.

\section{Distribution}

Lower peninsular Thailand and northern peninsular Malaysia. Distribution is shown in Fig. 19.

\section{Uvaria hahnii (Finet \& Gagnep.) J.Sinclair, Gard. Bull. Singapore 14: 44 (1955)}

Unona hahnii Finet \& Gagnep., Bull. Soc. Bot. France (Mém. 4 (2)) 53: 78 (1906); Desmos hahnii (Finet \& Gagnep.) Merr., Philipp. J. Sci. 10: 235 (1915); Melodorum hahnii (Finet \& Gagnep.) Bân, J. Biol. (Vietnam) 16(4): 8 (1994). 
Type: CAMBODIA. Kamchay Mountains, north-west of Kampot, 2 Feb. 1896, Hahn s.n. (lecto: P 00261139, isolecto: P 00261140-1, fide N.T.Bân, Fl. Vietnam 1: 41 (2000)); CAMBODIA. Kampot, 13 May 1904, Geoffray 385 (syn: P 00261138!); Kampot, Mar. 1874, Pierre 1786 (syn: P 00261144 !).

\section{Notes}

The narrow elongate petals and large wavy sepals and bracts of $U$. hahnii make it easy to distinguish from $U$. dulcis (peninsular Malaysia), its most similar-looking relative.

\section{Key to the varieties in continental Asia}

\footnotetext{
1. Petals $\geq \sim 35 \mathrm{~mm}$ long - Indochina and Myanmar 31a. U. hahnii var. hahnii Petals $\leq \sim 30 \mathrm{~mm}$ long - Kontum Province, Vietnam 31b. U. hahnii var kontumense
}

\section{1a. Uvaria hahnii (Finet \& Gagnep.) J.Sinclair var. hahnii}

Scandent shrub or woody climber. Young shoots subtomentose, becoming glabrous, bark black with deep striations. Leaves elliptic-narrowly obovate, subcoriaceous, apex acute-acuminate, base retuse, $\sim 100-160 \times 40-65 \mathrm{~mm}$, veins 9-12(-14); indument upper blade sparse becoming glabrous, hairs $3-5$-branched, fine, spreading, midrib hairs erect, 5+-branched, subtomentose becoming sparse, lower blade hairs mixed, smaller fine hairs 4-6-branched, sparse at first, becoming glabrous, larger ferruginous hairs erect, rare on blade, subtomentose on veins and midrib. Petiole $\sim 7 \times 1 \mathrm{~mm}$, black and warty, tomentose, later sparse or subglabrous. Inflorescence flowers solitary, leafopposed, peduncle 1-3.5 mm long, pedicel $~ 9-10 \mathrm{~mm}$ long, prominently striate, broadening up to $\sim 2$-mm-diameter near receptacle, hairs tomentose, many-branched, ferruginous, bracts ovate-acute, $\sim 15-20 \times 10-15 \mathrm{~mm}$, leaf-like with prominent palmate venation, outer-surface indument sparse, hairs 5+-branched, stocky, subtomentose along central vein, hairs on inner surface finer, evenly spread over surface. Calyx sepals 3 , valvate, ovate-lanceolate, $\sim 15 \times 10 \mathrm{~mm}$, large and leaflike with prominent central vein, flat or convex, reflexed at maturity, inner and outer indument as for bracts. Corolla 2 whorls of 3 petals, inner petals narrower than outer, petals creamy-white or yellow in colour, lanceolate-acute with prominent longitudinal veins, whorls unequal, outer petals $\sim 35-45 \times 10 \mathrm{~mm}$, base narrowed to $\sim 2 \mathrm{~mm}$, inner petals $\sim 35-45 \times 6-7 \mathrm{~mm}$, base narrowed to $\sim 1.5 \mathrm{~mm}$ with two $\sim 3$-mm-long marginal glands present, indument on outer surface of both whorls mixed, underlying tomentum of fine pale hairs overlain basally and along central axis by scattered 5+-branched erect ferruginous hairs, inner surface of both whorls tomentose, hairs fine, base somewhat glabrous on outer whorl, distinctly glabrous on inner whorl. Stamens numerous, $\sim 2.5 \mathrm{~mm}$ long, cuneate, connective apex convex, umbonate, bright orange in colour and distinctly pappillate, locules raised on short filament, varying in length. Carpels $\sim 20, \sim 3.5 \mathrm{~mm}$ long, ovary tomentose, narrowed into distinct neck below stigma, stigma $\sim 1 \mathrm{~mm}$ long, $\mathrm{U}$-shaped, hairy, ovaries $\sim 14$ in 2 rows. Fruits monocarps $\sim 4-8$, ovoid-oblong, $\sim 16-10 \times$
9-10.5 mm, minutely striate, tomentose, pericarp thick and soft at maturity, stipe short, $\sim 8 \mathrm{~mm}$ long; seeds $10-14$ in 2 rows, $\sim 7 \times 4 \times 2 \mathrm{~mm}$, compressed ovoid, smooth, aril $3 \times 2 \mathrm{~mm}$, often transversely compressed. (Fig. 1B.)

\section{Distribution}

Myanmar, Thailand, Cambodia and Laos. Distribution is shown in Fig. 14.

\section{1b. Uvaria hahnii var. kontumensis (Bân) C.Meade \&} J.Parn., comb. et stat. nov.

Melodorum kontumense Bân, Fl. Vietnam 1: 39 (2000).

Type: VIETNAM, Kontum Province, Moray Dak To, 3 Apr. 1978, Ly 430 (holo: VNM!).

Melodorum kontumense T.B.Nguyen in Pham-hoàng Hộ (ed.), Ill. Fl. Vietnam 1: 334 (1991), nom. inval.

Differs from the typical variety of the species in having subequal whorls with slightly shorter petals, and narrowly obovate and acuminate leaves with a subtomentose indument on both surfaces.

\section{Distribution}

Kontum Province, central Vietnam. Distribution is shown in Fig. 14.

\section{Uvaria dulcis Dunal, Monogr. Anonac. 90, t. 13 (1817)}

Anomianthus dulcis (Dunal) J.Sinclair, Gard. Bull. Straits Settlem. 3: 40 (1953).

Type: INDONESIA. Java, Ventenat hb., La Haye s.n. (lecto, here designated: G 00237861! (Herb. Delessert)).

Uvaria javana Dunal, Monogr. Anonac. 91, t. 14 (1817).

Type: INDONESIA. Uvaria javana, [Uvaria tomentosa? Roxb. t. 35 perquam affinis], Java, Ventenat hb., La Haye s.n. (lecto, here designated: G 00237862! (Herb. Delessert)).

Uvaria aurita Blume, Fl. Javae 21, 22: 15, pl. 2 (1830). Anomianthus auritus (Blume) Backer, Schoolfl. Java 23 (1911).

Type citation: '...in montosis Javae occidentalis...'.

Type: ?BO n.v. 'Uvaria aurita', C.L.Blume, Fl. Javae 21, 22: pl. 2 (1830) (lecto, here designated).

Uvaria heterocarpa Blume, Fl. Javae 21, 22: 41, pl. 17 (1830). Anomianthus heterocarpus (Blume) Zoll., Linnaea 29: 324 (1858).

Type citation: '...in interioribus provinciae Bantam, ... VAN HASSELT...'.

Type: ?BO n.v.

Type: ?BO n.v. 'Uvaria heterocarpa', C.L.Blume, Fl. Javae 21, 22: pl. 2 (1830) (lecto, here designated).

Uvaria pachychila Jovet-Ast, Suppl. Fl. Indochine 1: 62 (1938), nom. inval. pro syn.

Uvaria pachychila T.B.Nguyen in Phạm-hoàng Hộ (ed.), Ill. Fl. Vietnam 1: 307 (1991), nom. inval.

Sarmentose shrub or climber, reaching lengths of $30 \mathrm{~m}$ or more. Young shoots tomentose-subtomentose, becoming glabrous and striate with distinctive pale lenticels. Leaves 
elliptic-broadly elliptic-shortly obovate, apex acute, base subcordate-shortly cordate, $\sim 100-140 \times 45-60 \mathrm{~mm}$, membranous; veins $15-18$, frequently branching basally and medially; indument upper blade hairs 1-4+-branched, sparse becoming subglabrous, over midrib hairs erect, tomentose, $\sim 1 \mathrm{~mm}$ long, lower blade hairs 5+-branched, subtomentose, on veins and midrib hairs $\sim 10$-branched, ferruginous, stocky, tomentose towards petiole. Petiole tomentose, $\sim 3.5-5 \times 1 \mathrm{~mm}$. Inflorescence terminal, pendulous, flowers 1 to many, usually 2 or 3 , peduncle $\sim 5-10 \mathrm{~mm}$, pedicel $\sim 12-25 \mathrm{~mm}$, both ferruginotomentose, bracts broadly ovate, subtomentose, $\sim 2-3 \times$ $3.5-4.5 \mathrm{~mm}$, often wanting. Calyx sepals 3 , valvate, connate at base, $\sim 3.25 \times 4.5 \mathrm{~mm}$, ovate-acute, outer surface ferruginotomentose, inner surface subtomentose, hairs finer. Corolla 2 whorls of 3 petals, inner petals smaller than outer, petals white or yellow to pink in colour, broadly elliptical-acute at first, expanding to obovate-acute at maturity, outer petals 21.5 $\times 11.5 \mathrm{~mm}$, indument on outer surface mixed, underlying tomentum of fine pale hairs overlain by scattered larger rufous hairs usually gathered into distinct trace along central axis, inner surface basally glabrous, at apex hairs as for underlying tomentum on outer surface, inner petals $\sim 20.5 \times$ $10.5 \mathrm{~mm}$, outer surface tomentose, rufous hairs present but less numerous than on outer whorl, inner surface basally glabrous with two lateral orange-coloured glands inserted above the base. Stamens numerous, $\sim 1.5 \mathrm{~mm}$ long, connective broad and flattened, often forming lip on inner side, papillate, glabrous, locules raised on short filament. Carpels $\sim 25,2.5 \mathrm{~mm}$ long, hairy usually below stigma neck and along lateral ridges, stigma long and hairy with distinct neck, U-shaped, gynoecium forming distinct dome above stamens, ovules $\sim 14-16$. Fruit monocarps $\sim 10+$, oblong or shortly cylindrical, $\sim 10 \times 6.5 \mathrm{~mm}$, glabrous, green turning orange, then red, pericarp hard at first, juicy when ripe, becoming thin and flaky on drying, particularly in mature fruits, stipe $\sim 5-10 \mathrm{~mm}$; seeds 1-8, 5 × $4 \times 4 \mathrm{~mm}$, compressed-ovoid, raphe distinct, aril transversely compressed.

\section{Distribution}

Uvaria dulcis is often found in scrub vegetation; however, it is most common in disturbed moist forest where it can form large localised populations. It is common in the low-rainfall zones of north-eastern Thailand and central Java. Distribution is shown in Fig. 19.

\section{Notes}

The membranous obovate petals with conspicuous lateral glands on the inner whorl should be enough to distinguish this species from its closest relatives. Examination of the type material of $U$. dulcis and $U$. javana at Geneva showed that they are in fact the same species, explaining why Dunal's original descriptions of the two are almost identical. The name $U$. javana Dunal was misapplied to a different unnamed taxon from Java by Blume (1830). The type material for both $U$. aurita and $U$. heterocarpa is missing; thus, Blume's fullcolour illustrations from the original accounts are designated here as lectotypes.
33. Uvaria siamensis (Scheff.) L.L.Zhou, Y.C.F.Su \& R.M.K. Saunders, Syst. Biodiv. 7: 255 (2009)

Rauwenhoffia siamensis Scheff., Ann. Jard. Bot. Buitenzorg. 2: 23 (1885); Melodorum siamensis (Scheff.) Bân, Bot. Zhurn. (Moscow \& Leningrad) 39: 241 (1974).

Type: INDONESIA. '...cultivée dans notre jardin, dans lequel elle a été introduite par M. TEYSMANN, qui la trouva en Siam (Herb. Bog. no. 17785).' (?BO, n.v.).

Uvaria godefroyana Finet \& Gagnep., Bull. Soc. Bot. France (Mém. 4 (2)) 53: 71 (1906).

Type: CAMBODIA. Pursat, 11 June 1875, Godefroy/ Harmand 298 (lecto, here designated: P 00520753; isolecto: P 00520752!, P 00142663!); CAMBODIA. Angkor, 1875, Godefroy/Harmand 612 (syn: P 00252671!); CAMBODIA. Angkor, 1875, Godefroy/Harmand 621 (syn: P 00142664!).

Melodorum schefferi Pierre ex Finet \& Gagnep., Bull. Soc. Bot. France (Mém. 4 (2)) 53: 134, pl. 19a (1906).

Type: CAMBODIA. Bien Hoa Province, North, Chiao Xhay, Mar. 1877, Pierre 1772 (lecto, here designated: P 00373696!).

Shrub or small tree up to $2 \mathrm{~m}$, or climber up to $20 \mathrm{~m}$. Young shoots tomentose with large many-branched ferruginous hairs scattered over smaller, finer, less pigmented hairs, not quite obscuring bark, but nearly so, older wood becoming striate with prominent lenticels, subglabrous. Leaves (bluntly) lanceolate to elliptical, base retuse, apex narrowly-bluntly acute, $\sim 90-150 \times 30-40 \mathrm{~mm}$, thinly coriaceous, waxy texture above; veins 8-11(-14); indument glabrous above, except for sparse spreading stellate hairs on upper midrib, subtomentose below with underlying layer of fine, $\sim 6-8$-branched hairs overlain with scattered, thickened, many-branched hairs, especially on midrib and midrib base. Petiole tomentose, $\sim 3.5$ $\times 1.25 \mathrm{~mm}$. Inflorescence leaf-opposed or subopposed, flowers solitary or rarely 2 or 3 , peduncle subsessile, $\sim 1.5 \times 1.25 \mathrm{~mm}$, pedicel $\sim 8 \times 1.25 \mathrm{~mm}$, shortly and densely tomentose, hairs pale, overlain by scattered larger rufous hairs, deeply grooved or striate, basal and medial bracts ovate-acute, $\sim 2-5 \times 1-2 \mathrm{~mm}$, subtomentose, usually wanting. Calyx sepals 3 , valvate, opening early, very broadly ovate-acute, $\sim 3 \times 6 \mathrm{~mm}$, thickened and remaining incurved, base concave, outer surface indument as for pedicel, inner surface glabrous. Corolla 2 whorls of 3 petals, inner petals narrower than outer, petals yellow to white in colour, outer petals broadly ovate, $\sim 8 \times 8 \mathrm{~mm}$, inner petals broadly elliptical with constricted base, $\sim 8 \times 5.5 \mathrm{~mm}$, outer surface of both whorls densely tomentose, hairs fine, pale, inner surface of outer whorl tomentose at apex and on all margins, base glabrous, inner whorl glabrous at base, apex and apical margins tomentose. Stamens numerous, $\sim 1.5 \mathrm{~mm}$ long, cuneate, apex flat or shallowly convex, obscuring locules, rusty brown or orange in colour with waxy texture, covered with minute blunt papillae, locules raised on short filament. Carpels $\sim 10,3 \mathrm{~mm}$ long, ovaries covered with blunt stallate hair-scales, giving very finely warted appearance, neck between ovary and stigma covered with long stellate hairs, stigmas prominent, lobed, U-shaped, hairy, $\sim 1 \mathrm{~mm}$ long, forming distinct dome above stamens, often with copious exudate, ovules $\sim 4-8$. Fruit monocarps 2-10 (usually 3-6), globose or shortly oblong, $\sim 10-12 \times 8-9 \mathrm{~mm}$, very minutely 
tubercled with subtomentose indument of blunt stellate scales, outline of seeds often clear within pericarp, stipe subsessile, $\sim 3 \times$ $1 \mathrm{~mm}$, striate; seeds $\sim 2-4(-6)$, compressed hemi-ellipsoidal, $\sim 7 \times 4 \times 3 \mathrm{~mm}$. (Fig. 1E.)

\section{Distribution}

Although there are no records, it is probable that the species also occurs in the northern Malay States of Kelantan, Perlis and Kedah. Approximately 50\% of collections record U. siamensis as a shrub or small tree, most often occurring in disturbed or marginal forest vegetation, particularly bamboo scrub and forest. Distribution is shown in Fig. 16.

\section{Notes}

Uvaria siamensis and U. ferruginea have similar flowers, but the fruit, leaf and growth habits are very different. Uvaria siamensis is morphologically similar to $U$. hahnii, with the two species sharing fruit and leaf characters. The lectotypes selected present the best-preserved material from the originally cited sheets.

\section{Conflict of interest}

The authors declare that they have no conflict of interest.

\section{Acknowledgements}

This work was supported in part by the Oleg Polunen Memorial Fund, The Trinity Trust (University of Dublin) and the Royal Dublin Society. The authors thank Thomas Couvreur, David M. Johnson and Brendan Lepschi for their helpful comments in preparation of this manuscript.

\section{References}

Blume CL (1825) 'Bijdragen tot de flora van Nederlandsch Indie: 1.' (Lands Drukkerij: Batavia, Dutch East Indies)

Blume CL (1830) 'Flora Javae (Annonaceae).' (J. Frank: Brussels, Belgium)

Camin JH, Sokal RR (1965) A method for deducing branch sequences in phylogeny. Evolution 19, 311-326. doi:10.1111/j.1558-5646.1965.tb01722.x

Chatrou LW, Pirie MD, Erkens RHJ, Couvreur TLP, Neubig KM, Abbott JR, Mols JB, Maas JW, Saunders RMK, Chase MW (2012) A new subfamilial and tribal classification of the pantropical flowering plant family Annonaceae informed by molecular phylogenetics. Botanical Journal of the Linnean Soc. Bot. 169, 5-40. doi:10.1111/j.1095-8339.2012.01235.x

Couvreur TLP, Pirie MD, Chatrou LW, Saunders RMK, Su YCF, Richardson JE, Erkens RHJ (2011) Early evolutionary history of the flowering plant family Annonaceae: steady diversification and boreotropical geodispersal. Journal of Biogeography 38, 664-680. doi:10.1111/j.1365-2699.2010.02434.x

de Candolle AP (1824) 'Prodromus systematis naturalis regni vegetabilis. Vol. I.' (Treuttel \& Wurz: Paris, France)

de Lamarck JBAPM (1785). 'Encyclopédie Méthodique. Vol. 1.' (Chez Panckoucke later Chez H. Agasse: Paris, France)

Dunal MF (1817). 'Monagraphie de la famille des Anonacées.' (Treuttel \& Wurtz: Paris, France)

Edwards AWF, Cavalli-Sforza LL (1964). Reconstruction of evolutionary trees. In 'Phenetic and Phylogenetic Classification'. (Eds VH Heywood, J McNeill) Systematics Association publication number 6, pp. 67-76. (Systematics Association: London, UK)
Finet A, Gagnepain F (1906) Contribution à l'étude de la flore de l'Asia orientale. Fam. 5.: Anonacées. Bulletin de la Société botanique de France 53, Quatrième Série Mémoires 4(2), 55-170.

Forman LL (1997) Notes concerning the typification of names of William Roxburgh's species of Phanerogams. Kew Bulletin 52, 513-534. doi: $10.2307 / 4110285$

Fries RE (1955) Verstreute Beobachtungen hinsichtlich der famile Annonaceae. Arkiv för Botanik. Stockholm (Series 2) 3, 35-42. [Published in German]

Fries RE (1959) Annonaceae. In 'Die natürlichen Pflanzenfamilien Band 17a II'. (Eds A Engler, K Prantl) pp. 1-171. (Duncker \& Humblot: Berlin, Germany)

Hartl D, Clark A (2007). 'Principles of Population Genetics', 4th edn. (Sinauer Associates: Sunderland, MA, USA)

Hermann P (1717). Musaeum Zeylanicum. (Severinus: Leiden, Germany) Hooker JD, Thomson T (1855) ‘Flora Indica. Vol. 1.' (W Pamplin: London, UK)

Huber H (1985). Annonaceae. In 'A Revised Handbook to the Flora of Ceylon. Vol. 5'. (Ed. MD Dassanayake) pp. 1-75. (Amarind Publishing Co. Pvt. Ltd: New Delhi, India)

Jovet-Ast S (1938) Annonacées. In 'Supplément a la Flore Générale de L'Indochine.' Vol. 1'. (Ed. F Gagnepain) pp. 59-123. (Museum National d'Histoire Naturelle: Paris, France)

Jovet-Ast S (1940) Anonaceés nouvelles d'Indochine. Notulae Systematicae. Herbier du Muséum de Paris. Phanérogramie, Paris 9, 73-88. [Published in French]

Kessler PJA (1993) Annonaceae. In 'Families and Genera of Vascular Plants: 2. Flowering Plants: Dicotolydons. Magnoliid, Hamemelid and Caryophyllid families'. (Ed. K Kubitzki) pp. 93-129. (Springer Verlag: Berlin, Germany)

Kluge AG (1988) Parsimony in vicariance biogeography: a quantitative method and a greater Antillean example. Systematic Biology 37(4), 315-328. doi:10.1093/sysbio/37.4.315

Knowles L, Carstens B (2007) Delimiting species without monophyletic trees. Systematic Biology 56(6), 887-895. doi:10.1080/10635150701701091

Linnaeus CL (1753) 'Species plantarum. Vol. 1.' (Laurentii Salvii: Stockholm, Sweden)

Meade C (2001) A systematic revision of the Uvaria group (Annonaceae) in continental Asia. PhD Thesis, Trinity College, University of Dublin, Ireland.

Meade C (2005) A new species of Uvaria (Annonaceae) from Indochina. Adansonia 27(1), 17-20.

Meade C, Parnell J (1998) A revision of Uvaria L. in continental Southeast Asia: 1. Stamen and carpel structure. Annonaceae Newsletter 12, 47-55.

Meade C, Parnell J (2002) Neotypification of Uvaria hirsuta Jack. Taxon 51(4), 767-768. doi:10.2307/1555035

Meade C, Parnell J (2003) Multivariate analysis of leaf shape variation in the Uvaria group (Annonaceae). Botanical Journal of the Linnean Society 143, 231-242. doi:10.1046/j.1095-8339.2003.00223.x

Meade C, Hodkinson TR, Parnell J, Chalermglin P, Chase MW (2002) Revision of Uvaria L. in continental Southeast Asia: 2. Floral character evolution in the Uvaria L. group. Annonaceae Newsletter 13, 29-32.

Nguyễn TB (1974) Kriticheskie zametki o rodakh Melodorum Lour., Mitrella Miq., I Rauwenhoffia Scheff. (sem. Annonaceae Juss.). Botanicheskii Zhurnal, Moscow \& Leningrad 59, 237-245. [Published in Russian]

Nguyễn TB (2000) 'Thực Vật Chí Việt Nam, Flora of Vietnam, Vol. 1, Annonaceae.' (Science and Technics Publishing House: Hanoi, Vietnam)

Radford A, Dickinson W, Massey J, Bell C (1974). 'Vascular Plant Systematics.' (Harper \& Row: New York, NY, USA)

Roxburgh W (1832) 'Flora Indica, Vol. 2', 2nd edn. (W. Thacker \& Co.: Calcutta, India) 
Saunders RM (2010) Floral evolution in the Annonaceae: hypotheses of homeotic mutations and functional convergence. Biological Reviews of the Cambridge Philosophical Society 85, 571-591.

Saunders RM (2012) The diversity and evolution of pollination systems in Annonaceae. Botanical Journal of the Linnean Society 169, 222-244. doi:10.1111/j.1095-8339.2011.01208.x

Sealy JR (1956) The Roxburgh flora Indica drawings at Kew. Kew Bulletin 11, 297-399. doi:10.2307/4109049

Sinclair J (1951) Notes on Bornean Annonaceae. Sarawak Museum Journal 5(3), 597-609.

Sinclair J (1955) A revision of the Malayan Annonaceae. Gardens' Bulletin 14(2), 149-516.

Swofford DW (2002) 'PAUP*. Phylogenetic Analysis Using Parsimony (*and other methods). Version 4.' (Sinaur Associates: Sunderland, MA, USA)

Thomas DC, Chatrou LW, Stull GW, Johnson DM, Harris DJ, Thongpairoj U, Saunders RMK (2015) The historical origins of palaeotropical intercontinental disjunctions in the pantropical flowering plant family Annonaceae. Perspectives in Plant Ecology, Evolution and Systematics 17(1), 1-16. doi:10.1016/j.ppees.2014.11.001

Tsou C-H, Johnson DM (2003) Comparative development of aseptate and septate anthers of Annonaceae. American Journal of Botany 90, 832-848. doi:10.3732/ajb.90.6.832
Utteridge TMA (2000) Revision of the genus Cyathostemma (Annonaceae). Blumea 45(2), 377-396.

Van Heusden ECH (1992) Flowers of Annonaceae: morphology, classification and evolution. Blumea 7(Suppl.), 1-218.

van Rheede Tot Draakenstein HA (1679) 'Hortus Indicus Malabaricus, Vol. 2.' (Sumptibus Joannis van Someren etc.: Amsterdam, Netherlands)

Zhou L, Su YCF, Saunders RMK (2009) Molecular phylogenetic support for a broader delimitation of Uvaria (Annonaceae), inclusive of Anomianthus, Cyathostemma, Ellipeia, Ellipeiopsis and Rauwenhoffia. Systematics and Biodiversity 7, 249-258. doi:10.1017/S1477200009003028

Zhou L, Su YCF, Chalermglin P, Saunders RMK (2010) Molecular phylogenetics of Uvaria (Annonaceae): relationships with Balonga, Dasoclema and Australian species of Melodorum. Botanical Journal of the Linnean Society 163, 33-43. doi:10.1111/j.1095-8339.2010.01045.x

Zhou L, Su YCF, Thomas DC, Saunders RMK (2012) 'Out-of-Africa' dispersal of tropical floras during the Miocene climatic optimum: evidence from Uvaria (Annonaceae). Journal of Biogeography 39, 322-335. doi:10.1111/j.1365-2699.2011.02598.x

Handling editor: Peter Wilson 